\title{
ELECTRODE GEOMETRY EFFECTS IN AN ELECTROTHERMAL PLASMA MICROTHRUSTER
}

\author{
A Thesis \\ presented to \\ the Faculty of California Polytechnic State University, \\ San Luis Obispo
}

In Partial Fulfillment

of the Requirements for the Degree

Master of Science in Aerospace Engineering

by

Harrison King

June 2018 
(C) 2018

Harrison King

ALL RIGHTS RESERVED 


\section{COMMITTEE MEMBERSHIP}

TITLE:

Electrode Geometry Effects in an Electrothermal Plasma Microthruster

AUTHOR: Harrison King

DATE SUBMITTED: June 2018

COMMITTEE CHAIR: Amelia Greig, Ph.D.

Professor of Aerospace Engineering

COMMITTEE MEMBER: Dianne Deturris, Ph.D.

Professor of Aerospace Engineering

COMMITTEE MeMBeR: Graham Doig, Ph.D.

Professor of Aerospace Engineering

COMMITTEe MEMBER: Ian Johnson, Ph.D.

Control Systems Engineer, SSL 


\begin{abstract}
Electrode Geometry Effects in an Electrothermal Plasma Microthruster Harrison King
\end{abstract}

Nanosatellites, such as Cubesats, are a rapidly growing sector of the space industry. Their popularity stems from their low development cost, short development cycle, and the widespread availability of COTS subsystems. Budget-conscious spacecraft designers are working to expand the range of missions that can be accomplished with nanosatellites, and a key area of development fueling this expansion is the creation of micropropulsion systems. One such system, originally developed at the Australian National University (ANU), is an electrothermal plasma thruster known as Pocket Rocket (PR). This device heats neutral propellant gas by exposing it to a Capacitively Coupled Plasma (CCP), then expels the heated gas to produce thrust. Significant work has gone towards understanding how PR creates and sustains a plasma and how this plasma heats the neutral gas. However, no research has been published on varying in the device's geometry. This thesis aims to observe how the size of the RF electrode affects PR operation, and to determine if it can be adjusted to improve performance. To this end, a thruster has been built which allows the geometry of the RF electrode to be easily varied. Measurements of the plasma density at the exit of this thruster with different sizes of electrode were then used to validate a Computational Fluid Dynamics (CFD) model capable of approximately reproducing experimental measurements from both this study and from the ANU team. From this $\mathrm{CFD}$, the number of argon ions in the thruster was found for each geometry, since collisions between argon ions and neutrals are primarily responsible for the heating observed in the thruster. A geometry using a $10.5 \mathrm{~mm}$ electrode was observed to produce a $23 \%$ increase in the quantity of ions produced compared to the baseline 5 $\mathrm{mm}$ electrode size, and a $3.5 \mathrm{~mm}$ electrode appears to produce $88 \%$ more ions. 


\section{ACKNOWLEDGMENTS}

Thanks to:

- Dr. Amelia Greig, for being patient with my stupid questions and letting me test and learn from my bad ideas.

- Dr. Ian Johnson, Dr. Graham Doig, and Dr. Dianne DeTurris for their time, feedback, and helpful suggestions.

- My family, for their emotional and material support. Thanks especially for the cookies.

- Alaina, for tolerating my panicked pre-deadline frenzies, keeping me company while I wrote indoors on nice days, and waking up early on weekends to sit with me in the lab.

- Charlie, Alec, and Nick, for being excited to help me out in the lab and willing to show up at a moment's notice. 


\section{TABLE OF CONTENTS}

LIST OF TABLES . . . . . . . . . . . . . . . . . . . . . . . . . . . . . . . . . . . . viii
LIST OF FIGURES
CHAPTER

1 Introduction . . . . . . . . . . . . . . . . . . 1

1.1 Micro-Propulsion . . . . . . . . . . . . . . . . 1

1.2 Plasma Physics . . . . . . . . . . . . . . . . 3

1.2.1 What is Plasma? ................. 3

1.2.2 Plasma Generation ................ 5

1.2.3 Secondary Electrons . . . . . . . . . . . . . . 7

1.2.4 Plasma Collisions . . . . . . . . . . . . . . . . 10

1.2.5 Sheath Enhancement . . . . . . . . . . . . . 12

1.3 Pocket Rocket . . . . . . . . . . . . . . . . . 13

1.4 Thesis Scope. . . . . . . . . . . . . . . . 16

2 Apparatus and Methodology . . . . . . . . . . . . . . 17

2.1 Experimental Apparatus . . . . . . . . . . . . . . . 17

2.1.1 Testing Chamber and Plumbing . . . . . . . . . . 17

2.1.2 Electrical Routing. . . . . . . . . . . . . . 19

2.1.3 Thruster Hardware . . . . . . . . . . . . . . . . . 20

2.2 Data Collection . . . . . . . . . . . . . . . 24

2.2.1 Langmuir Probe. . . . . . . . . . . . . . . 24

2.2.2 Optical Diagnostics . . . . . . . . . . . . . . . 28

2.3 Computational Fluid Dynamics Model . . . . . . . . . . . . . 29

2.3.1 CFD Model Description . . . . . . . . . . . . . 30

2.3.2 Model Verification . . . . . . . . . . . . . . 35

2.3.3 Preliminary Validation . . . . . . . . . . . . 36

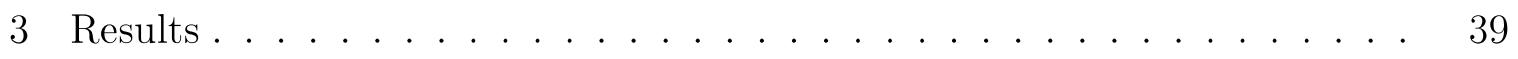

3.1 Voltage Measurement . . . . . . . . . . . . . . . . 39

3.2 Langmuir Probe Measurements . . . . . . . . . . . . . . . . 40 
3.3 Optical Diagnostics . . . . . . . . . . . . . . . . . . . . . . 44

3.4 CFD Validation . . . . . . . . . . . . . . . . . . 45

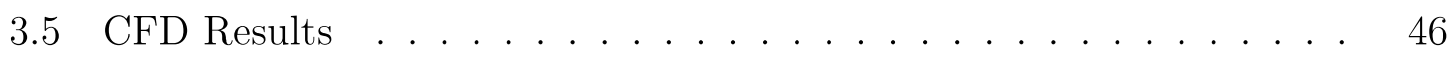




APPENDICES

A Thruster Operation Procedures . . . . . . . . . . . . 61

B Simulation Automation Script . . . . . . . . . . . 66

C Model Setup Script . . . . . . . . . . . . . . . . . . . . 68 


\section{LIST OF TABLES}

Table

1.1 Micropropulsion Systems . . . . . . . . . . . . . . 2

$2.1 \quad$ Electrode Dimensions $(\mathrm{mm}) \ldots \ldots \ldots 22$ 


\section{LIST OF FIGURES}

Figure $\quad$ Page

$1.1 \quad$ Four Phases of Matter . . . . . . . . . . . . . . 4

1.2 Features of Plasma Near a Wall (Not to Scale) . . . . . . . . . 5

1.3 Secondary Electron Emission Process . . . . . . . . . . . . . . . 8

1.4 Charge Exchange Collision Process . . . . . . . . . . . . . . 11

1.5 Capacitive Discharge Equivalent Circuit . . . . . . . . . . . 13

$1.6 \quad$ Pocket Rocket Device $[34] \ldots \ldots$. . . . . . . . . . . . . . 14

$2.1 \quad$ Plumbing Layout . . . . . . . . . . . . . . . . . . . . 18

2.2 Simplified Power System Schematic . . . . . . . . . . . . . . . 20

2.3 Selected Discharge Tubes _. . . . . . . . . . . . . . . 21

2.4 Thruster Diagram . . . . . . . . . . . . . . . . 22

2.5 Experimental Apparatus . . . . . . . . . . . . . . . . 23

2.6 Langmuir Probe Electrical Schematic . . . . . . . . . . . . . . 25

2.7 Linear Fit to Ion Current - Typical Example . . . . . . . . . . . . . 26

2.8 Exponential Fits to Determine Electron Temperature . . . . . . . . 27

2.9 Plume Photographs . . . . . . . . . . . . . . . . 29

2.10 Full Simulation Domain _. . . . . . . . . . . . . . . 30

2.11 Thruster Domain Closeup With Dimensions (mm) . . . . . . . . 32

2.12 CFD Mesh . . . . . . . . . . . . . . . . . 32

2.13 Discharge Tube Mesh . . . . . . . . . . . . . . 33

2.14 Grid Convergence Results . . . . . . . . . . . . . . . . 36

2.15 Wall Convergence . . . . . . . . . . . . . 37

2.16 Plasma Density along Thruster Axis . . . . . . . . . . . . 38

3.1 Measured Electrode Voltage Vs Electrode Width . . . . . . . . . 40

3.2 First Two Langmuir Data Sets: $1 \sigma$ Error Bars . . . . . . . . . . . . 41

3.3 First Three Langmuir Data Sets: $1 \sigma$ Error Bars . . . . . . . . . . . 42

3.4 Langmuir Probe Data with True Error Bars . . . . . . . . . . . 43

3.5 Optical Versus Langmuir Probe Results . . . . . . . . . . . . . . . 44 
$3.6 \quad$ CFD Validation . . . . . . . . . . . . . . . . . . 45

3.7 Total Ion Quantity vs Electrode Width . . . . . . . . . . . . 47

3.8 Comparison of Electron Densities . . . . . . . . . . . . . 48

3.9 Comparison of Electron Temperatures . . . . . . . . . . . . 49

3.10 Total Excited Neutral Quantity vs Electrode Width . . . . . . . . 51

3.11 Comparison of Excited Atom Density . . . . . . . . . . . . 52 


\section{Chapter 1}

\section{INTRODUCTION}

Nanosatellites, and in particular Cubesats, have enjoyed ever-increasing popularity in recent years thanks to the many benefits associated with their small size. Cubesats are built on a fundamental $10 \mathrm{~cm}^{3}$ unit, or "U", and consist of one or more of these unit volumes combined together. The most common are $1 \mathrm{U}, 3 \mathrm{U}$, and $6 \mathrm{U}$ satellites. Cubesats are well-known to be inexpensive and quick to develop, allowing programs to go from conception to launch in a matter of months [1]. Until very recently, though, it was not feasible for Cubesats to carry a propulsion system, as there were no flight-tested systems that were effective at a nanosatellite scale. However, rising interest in this sector of satellite development has driven a surge of investment in propulsion devices to broaden the range of missions available to nano-sats. There are now COTS propulsion solutions marketed to nano-sat designers tackling a broad range of applications, varying from counteracting orbital perturbations to performing asteroid rendezvous. Systems like these are collectively known as micro-propulsion.

\subsection{Micro-Propulsion}

Due to the broad range of mission applications for micropropulsion systems, there is a correspondingly broad variety of the devices themselves. Some missions require higher

thrust maneuvers with less total impulse, while some benefit from maximum efficiency and are less sensitive to the amount of thrust provided. Thruster types such as cold gas and monopropellant designs excel in relatively high thrust applications, while a variety of electric propulsion options are being investigated for their high efficiencies. A range of commercial and purpose-built micropropulsion solutions is shown below 
in Table 1.1, sorted approximately by how efficiently they use propellant $\left(\mathrm{I}_{\mathrm{sp}}\right)$.

Table 1.1: Micropropulsion Systems

\begin{tabular}{|c|c|c|c|c|c|c|c|}
\hline Name & Type & Isp (s) & Thrust $(\mathrm{mN})$ & Power $(\mathrm{W})$ & Mass (kg) & $\begin{array}{l}\text { Cubesat } \\
\text { Units }\end{array}$ & $\begin{array}{l}\text { Total } \\
\text { Impulse }(\mathrm{N} \cdot \mathrm{s})\end{array}$ \\
\hline NANOPS [2] [3] & cold gas & 47.6 & 35 & $<3$ & & & \\
\hline FMMR [4] & resistojet & 65 & 1.2 & 3.2 & & & \\
\hline $\begin{array}{l}\text { Pocket Rocket } \\
{[5][6]}\end{array}$ & electrothermal & $<100$ & 2.4 & 32 & & $1 \mathrm{U}$ & \\
\hline ArgoMoon [7] & cold gas & 126 & 100 & 4.3 & 2.065 & $1.3 \mathrm{U}$ & 783 \\
\hline $\begin{array}{l}\text { Busek Micro } \\
\text { Resistojet [8] }\end{array}$ & resistojet & 150 & $2-10$ & $3-15$ & 1.25 & $1 \mathrm{U}$ & 404 \\
\hline HYDROS-C [9] & electrochemical & 310 & $1.2(\mathrm{avg})$ & $5-25$ & 2.6 & $2 \mathrm{U}$ & 2151 \\
\hline BmP-220 [10] & PPT & 536 & $.02-.14$ (avg) & $1.5-7.5$ & 0.5 & $0.34 \mathrm{U}$ & 220 \\
\hline BET-1mN [11] & electrospray & 800 & 0.7 & 15 & 1.15 & $1 \mathrm{U}$ & 605 \\
\hline $\begin{array}{l}\text { Accion } \\
\text { Tile } 5000 \text { [12] }\end{array}$ & electrospray & 1500 & 1.5 & 30 & 1.1 & 1.25 & $5000+$ \\
\hline $\begin{array}{l}\text { DSI Comet-1-300 } \\
\text { [13] }\end{array}$ & resistojet & $150-175$ & $3.6-4$ & 10 & 0.65 & $1.5 \mathrm{U}$ & 514.5 \\
\hline $\begin{array}{l}\text { IMF Nano } \\
\text { Thruster [14] }\end{array}$ & FEEP & $2000-5000$ & $.01-.5$ & 35 & 0.87 & $0.75 \mathrm{U}$ & $5000+$ \\
\hline $\begin{array}{l}\text { PhaseFour } \\
\text { RFT [15] }\end{array}$ & electromagnetic & $200-900$ & $1-16.5$ & $40-120$ & 2 & $1 \mathrm{U}$ & $1000-4400$ \\
\hline BGT-X5 [16] & monopropellant & $220-225$ & 500 & 20 & 1.5 & $1 \mathrm{U}$ & 565 \\
\hline
\end{tabular}

There are, of course, other factors that system integrators must consider when selecting a propulsion solution, such as system cost, system complexity/reliability, propellant toxicity/handling concerns, and more. This paper is centered around an effort to improve the performance of the Pocket Rocket thruster, listed near the top of the table due to the relatively low efficiency of its current configuration. The Pocket Rocket device is worth pursuing despite the current performance deficit because of the simplicity of its construction, which will allow it to become an inexpensive and reliable thruster. In short, the Pocket Rocket accelerates a neutral propellant gas by heating it with a plasma discharge. To better understand the operation of this device, 
however, a brief overview of plasma physics is required.

\subsection{Plasma Physics}

This section will explore the aspects of plasma physics required to describe the behavior of the Pocket Rocket, from the very basics up through some more detailed mechanisms.

\subsubsection{What is Plasma?}

Plasma, commonly known to be the fourth state of matter, is created when the particles of a gas become ionized. This is shown in Figure 1.1, where the four main phases are graphically compared. The separation of plasma into oppositely charged particles gives rise to several unique and defining properties of plasmas. These behaviors are caused by the phenomena known as "collective effects", which describe how charged particles in plasma influence and are influenced by the electromagnetic fields given off by other charged particles in the plasma and outside sources [17]. The most fundamental of these effects describes broadly how plasmas maintain roughly equal numbers of positive and negative particles distributed through the discharge, and is known as quasineutrality, or macroscopic neutrality. While plasmas are rarely perfectly neutral, the attraction between the positive and negative plasma particles prevents the numbers of either species from significantly increasing or decreasing relative to the other, thus keeping the plasma bulk nearly neutral, or quasineutral.

If a charged disturbance is placed in the plasma volume, it will attract oppositely charged particles, which rapidly attenuate the field disturbance and shield the bulk of the plasma from it. This means that, once this shielding distance has been exceeded, the potential within the plasma remains constant throughout the plasma bulk. This shielding distance is another fundamental property of plasmas, and is described in 


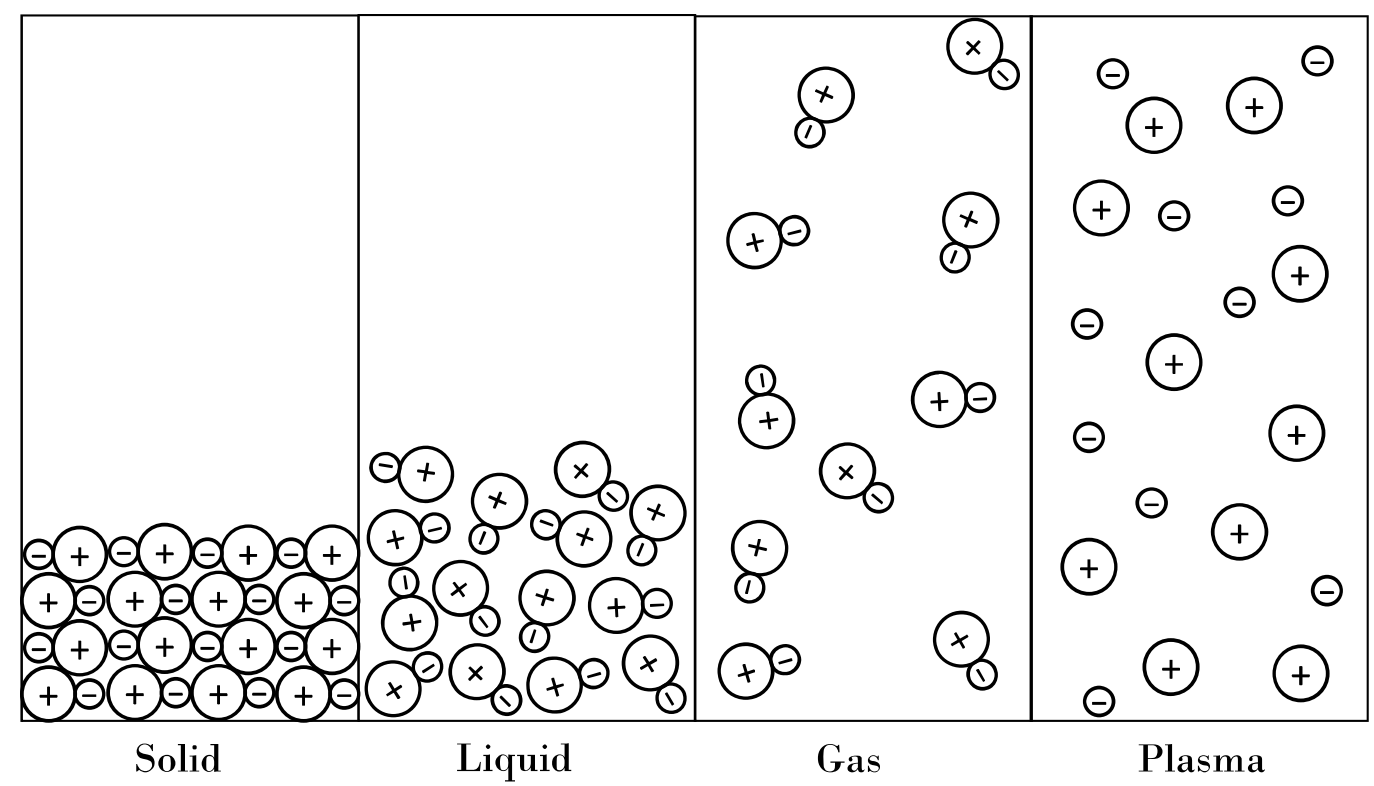

Figure 1.1: Four Phases of Matter

terms of the Debye length. The Debye length is determined by a simple formula that relates the plasma temperature and density to give the approximate scale over which shielding will occur, shown in equation 1.1 below.

$$
\lambda_{d}=\sqrt{\frac{\epsilon_{0} k T_{e}}{n_{e} e^{2}}}
$$

Here, $\lambda_{d}$ is the Debye length, $\epsilon_{0}$ is the permittivity of free space, $k$ is Boltzmann's constant, $T_{e}$ is the electron temperature, $n_{e}$ is the electron density, and $e$ is the fundamental charge. Comparing the Debye length of a sample to the size of its container is a useful metric for determining whether or not a sample can be treated as a plasma. If the container is not sufficiently larger than the Debye length, the sample will not be able to shield itself from the effects of the container walls to maintain quasineutrality, and therefore lacks the main defining property of a plasma [17] [18].

The area of the plasma that takes on this shielding role is known as the sheath. Sheaths are typically several Debye lengths in size [19]. Figure 1.2 illustrates the key features of a sheath by plotting potential against distance from the wall. The sheath itself is the area of steepest potential gradient, but is separated from the plasma 




\section{Figure 1.2: Features of Plasma Near a Wall (Not to Scale)}

bulk by the pre-sheath. The point separating the sheath and pre-sheath is the Bohm point, or sheath edge. Because all ions that enter the sheath must cross through the pre-sheath, all ions crossing the Bohm point will have some minimum velocity derived from the potential drop across the pre-sheath. The expression for this velocity, known as the Bohm velocity, is given below [20].

$$
V_{B}=\sqrt{\frac{K T_{e}}{M_{i}}}
$$

Here, $V_{B}$ is the Bohm velocity, $M_{i}$ is the ion mass, and $K$ and $T_{e}$ are Boltzmann's constant and the electron temperature as before. This will later become useful in working out the density of a plasma based on how many particles cross through a sheath, but this will be discussed in detail in section 2.2.1.

\subsubsection{Plasma Generation}

Plasmas are mainly generated in one of two ways. The first is to heat a gas up to the point that collisions between gas particles have enough energy to ionize each other. 
These plasmas exist naturally, such as in the sun and in ordinary flames. Under these conditions, the ion temperature and electron temperature in the plasma will be similar, since the entire sample is heated to generate the required ionization. In the laboratory, however, this approach is often impractical, especially since there is a much easier way to create samples of plasma. Exposing a gas to strong electric fields can accelerate the small number of ever-present free electrons in the gas to sufficient velocities to ionize any neutrals with which they collide. This in turn frees up more electrons to be accelerated by the electric field, creating a cascading effect until the rate at which the particles in the sample neutralize each other is matched by the rate at which they are ionized by the fast-moving electrons. This gas breakdown can be initiated with either $\mathrm{AC}$ or $\mathrm{DC}$ fields, but $\mathrm{AC}$ radiofrequency (RF) fields will be used in this study. In these types of plasmas, the electrons are much hotter (faster) than the ions, since their lower mass allows them to be accelerated much more quickly by the applied electric field [19]. Plasmas of this variety require external power to be applied continuously, and appear to vanish instantly when power is removed [17].

These laboratory plasmas, as they're known, can be further divided based on how the electromagnetic energy that sustains them is coupled into the plasma volume. In order of coupling strength, there are Capacitively Coupled Plasmas (CCPs), Inductively Coupled Plasmas (ICPs), and helicon wave coupled plasmas [21]. Although CCPs are known to be weaker than plasmas generated with the other coupling modes, often only reaching a few percent ionization instead of up to nearly $100 \%$ [22], they have a strong advantage in their simplicity. They can operate over a very wide range of frequencies and do not require specialized antennas to be designed; only simple plate electrodes are needed [23].

Despite the simplicity of their construction, though, CCPs still display some nuances in their behavior. This mainly arises from the several ways in which an oscillating, capacitively coupled field can accelerate electrons. If the electrons responsible 
for ionizing collisions in the plasma are found throughout the plasma bulk, the CCP is said to operate in alpha mode [23]. One way for these bulk electrons to gain sufficient energy to cause ionizations is for them to get caught up in a potential well that travels with the applied electromagnetic wave, which then transfers wave energy to the electrons in a process known as Landau damping [17]. Alternatively, electrons can be emitted when ions strike the walls of the discharge chamber in a process called ion-induced secondary electron emission. Because a sheath necessarily develops around the powered electrode to maintain quasineutrality, a significant potential gradient can exist between the negatively charged wall and the positively charged plasma bulk, which accelerates the ions that cross the sheath boundary towards the wall. These ions gain sufficient energy to have a chance at generating secondary electrons, which are then accelerated back into the plasma bulk by the same sheath field. Once they cross out of the sheath, these electrons can have high enough energies to ionize one or more neutral atoms, thus sustaining the discharge. CCPs that mainly couple power to the discharge through this mechanism are said to be operating in gamma mode [23]. Because the thruster used in this study operates in gamma mode, it will be important to cover the mechanics of secondary electron emission in greater detail.

\subsubsection{Secondary Electrons}

Ion-induced secondary electron emission occurs by way of a three step process that depends on various physical and chemical properties of the target material, and is shown in Figure 1.3. First, the incoming ion must ionize an atom in the target surface to release an electron [24] [25]. This is where the first material dependency appears; metals with lower work functions are more easily ionized, and therefore more likely to complete this first step. For insulators and semiconductors, the ionization energy is the sum of the electron affinity and band gap, so materials with lower totals of these 
quantities are more easily ionized, but will almost always be more difficult to ionize than conductive materials [24].



Figure 1.3: Secondary Electron Emission Process

Second, the freed electron may traverse the wall material. This stage is mainly important for higher energy collisions, as a higher energy impact will result in a freed electron with more energy, which can create a cascade of ionization as it traverses the material [24]. Such cascades of ionizing collisions explain why high-energy particles are able to yield multiple secondary electrons from a single impact. The interactions electrons undergo as they traverse the wall material can become very complex, but because the collisions in the Pocket Rocket environment are generally too weak to cause multiple ionizations of the wall material, the details of these interactions will be omitted. This traversing stage is also important in cases where an incoming ion penetrates some distance into the surface before engaging in an ionizing collision, as the freed electron must traverse back out to the surface before it can be emitted [25].

In the third and final stage, the freed electrons must leave the wall material. This requires them to traverse the barrier at the material's surface formed by the 
particular binding energy of the material [24]. It is because of this third step that metals typically have lower yields of secondary electrons; their larger binding energy compared to insulators is a larger factor than their lower ionization energies [24].

Since each stage in this process is influenced by different material properties, various combinations of surface preparations, films, and base materials can significantly change the secondary electron yield $(\gamma)$ of a surface [26]. Even variations in the crystal structure of a single compound can have significant effects on the $\gamma$ observed [27]. Values of $\gamma$ are also strongly dependent on the energy of the impacting particles, and increase nonlinearly as particle energy increases [24] [26] [25]. Since there are no published studies of $\gamma$ for sintered alumina when struck by low energy argon ions, the $\gamma$ of the thruster walls was used as a fitting parameter to match simulations to experiments. Yields used are within the same range as those measured for argon ion bombardment of $\mathrm{MgO}(0.02-0.1)$, an oxide much more heavily studied due to its use in plasma display panels [27] [28]. This range was deemed valid as $\mathrm{Al}_{2} \mathrm{O}_{3}$ and $\mathrm{MgO}$ have been determined to have similar behavior in capacitively coupled noble gas discharges [29].

Since only a fraction of the ions accelerated into the discharge walls manage to use their energy to ionize a wall atom, the remaining impacts must transfer their energy somewhere else. For the most part, this remaining energy is simply absorbed by the walls as heat. This heating is called ion bombardment heating [20], and is one of the ways the energy stored in a plasma can be used to heat its environment. It is also responsible for the observed increase in Pocket Rocket exhaust temperature over timescales of several minutes [5]. 


\subsubsection{Plasma Collisions}

In addition to collisions against walls, collisions within the plasma itself are very important to how the Pocket Rocket operates. The most important of these collision types was touched upon in Section 1.2.2, in which an electron with sufficient energy impacts a neutral and tears off its outermost valence electron, ionizing it. These ionizing collisions are what sustain the plasma. The opposite of this is called recombination, in which an ion and electron combine to form a neutral [18]. This process creates hot neutral atoms, but is a relatively rare occurrence, since the majority of ions and electrons are moving too quickly to stick together.

A more common source of hot neutrals is ion-neutral charge exchange collisions. In these interactions, a fast-moving ion passes close to a neutral atom, stealing its outermost valence electron in the process [18] [20]. The first particle retains all or most of its energy through the exchange, becoming a hot neutral [20]. The newly ionized slow particle can now be influenced by the electric fields applied to the plasma, so it can be heated and take part in further collisions. The charge exchange collision process is shown in Figure 1.4 with the color of each particle denoting its temperature. Since this process does not require the particles to collide, but only pass near each other, it occurs quite frequently compared to other collisional processes [20], and is the dominant mechanism by which the Pocket Rocket heats neutral gas [30].

Lastly, excitation collisions play a small role in the behavior of the discharge as well. When an electron hits a neutral without enough energy to cause ionization, it can instead raise the outermost valence electron one or more energy levels [18]. An atom in this state is said to be excited. From here, the excited atom can either return to its ground state by emitting a photon, or undergo further collisions. If the atom returns to its ground state, the energy lost as the electron drops from a higher energy level to the lowest empty level is released as a photon, the wavelength of which 


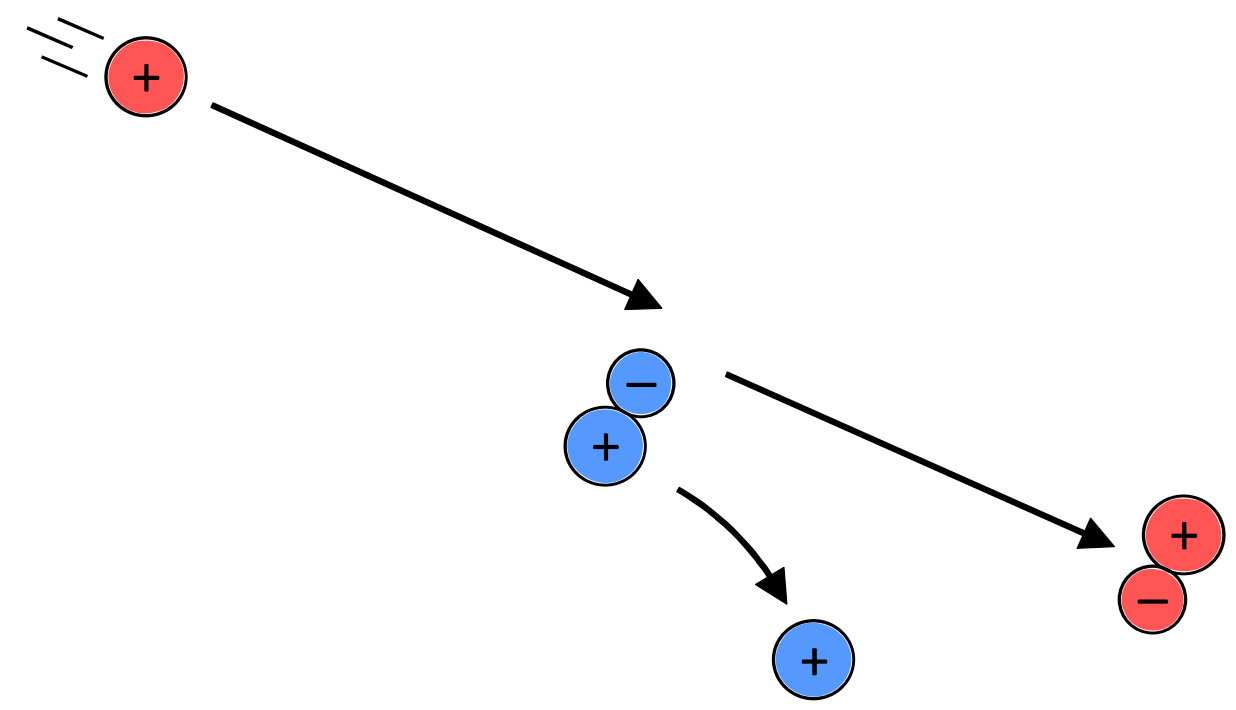

\section{Figure 1.4: Charge Exchange Collision Process}

corresponds to the quantity of energy released. Since each element has a unique arrangement of electrons, and therefore a unique set of possible electron energy level jumps, the wavelengths of emitted photons can act as an optical fingerprint to identify different elements. If the atom instead undergoes further collisions before releasing the excitation energy, the elevated electron can be given more energy, possibly leaving the atom altogether. This process is known as stepwise ionization [18].

These various types of excitation collisions were described as occurring between electrons and neutrals, but can occur between electrons and ions as well. If an ion becomes excited, it can also decay by photon emission or undergo further collisions to become doubly ionized. Since ions have a different configuration of energy levels than their neutral counterparts, they can be identified separately from excited neutrals based on their unique spectral fingerprint. This will become useful during experiments as a way to measure the relative concentrations of ions between various experimental setups.

Though useful for diagnostic reasons, these excitation collisions are not desirable in most plasma discharges. Since the optical energy emitted by decaying excited atoms 
is typically not re-absorbed by the discharge, it escapes as wasted energy. Increasing the electron temperature in a discharge will increase the number of ionizing collisions relative to excitation collisions, and can be an important consideration in improving the efficiency of a plasma discharge [20].

\subsubsection{Sheath Enhancement}

Since some of the collision modes described above are more desirable than others, it is often desirable to influence how many of each occur. This can be done by adjusting the electron energy distribution, since the energy of the electron involved in a collision strongly influences what type of collision takes place [18]. In the case of a CCP, where the electrons responsible for sustaining the discharge get their energy from the potential gradient through the electrode sheaths, changing the voltage difference between the plasma bulk and an electrode directly changes the sheath potential. One way to do this is simply to change the voltage being applied to the electrode by adjusting the power supply, but this also affects the power consumed by the device. With a constant supplied power, the equivalent circuit behavior of the discharge must be exploited to change the sheath potential. This equivalent circuit is shown in Figure 1.5 .

As illustrated in the diagram, the gaps between the electrode and the plasma bulk (which contains the insulating dielectric and sheath) act as capacitors through which energy is transferred to the discharge [31]. The voltage drop across each of these capacitive junctions is proportional to the impedance of the junction as a percentage of the total impedance of the system [31]. Therefore, increasing the impedance of one junction (or decreasing the impedance of its counterpart) will increase the voltage drop across that junction. The simplest method to adjust the impedance of either junction is to change its area; increasing electrode area decreases impedance, and 


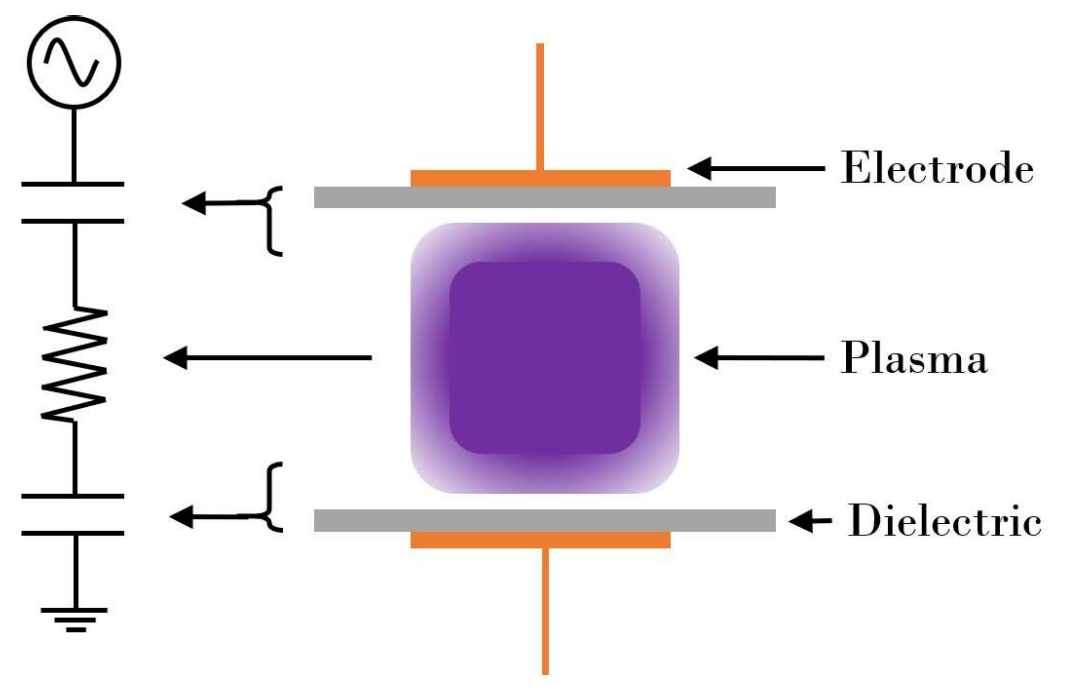

Figure 1.5: Capacitive Discharge Equivalent Circuit

decreasing area will add impedance. This relationship can be expressed as Equation $1.3[31]$.

$$
\frac{V_{R F}}{V_{G N D}}=\frac{C_{G N D}}{C_{R F}}=\frac{A_{G N D} S_{R F}}{A_{R F} S_{G N D}}
$$

Here, $C$ is the capacitance of the junction, $V$ is the voltage across the junction, $A$ represents electrode area, and $S$ is the distance between the electrode and plasma bulk, which includes the sheath and dielectric wall thicknesses. The subscript $R F$ denotes the RF electrode, and GND denotes the ground electrode. Also, even though Figure 1.5 and Equation 1.3 use only two electrodes, an arbitrary number of separate surfaces can be used on a physical device to collectively act as either one of the illustrated junctions.

\subsection{Pocket Rocket}

Pocket Rocket is an electrothermal thruster originally developed at the Australian National University (ANU) [32]. The device uses a capacitively coupled plasma discharge to heat a neutral gas above its storage temperature to produce higher thrust than a comparable cold gas setup [6]. At the core of the device is an alumina tube 
that contains the hot discharge, around which three electrodes are mounted. In the center is the driven RF electrode, and on either end sits a ground electrode that is extended outwards to meet the thruster housing. The electrodes are separated from one another by a Macor glass cylinder, which also helps trap heat in the discharge tube [5]. Upstream of the alumina discharge tube is the aluminum plenum chamber, which allows the propellant gas arriving through the feed line to settle before being used. The thruster ends at the downstream end of the discharge tube, which sits flush with the flat aluminum aft plate. This plate and the plenum both act to increase the area of the thruster's ground electrodes, which enhances the sheath around the driven electrode inside the thruster [5].

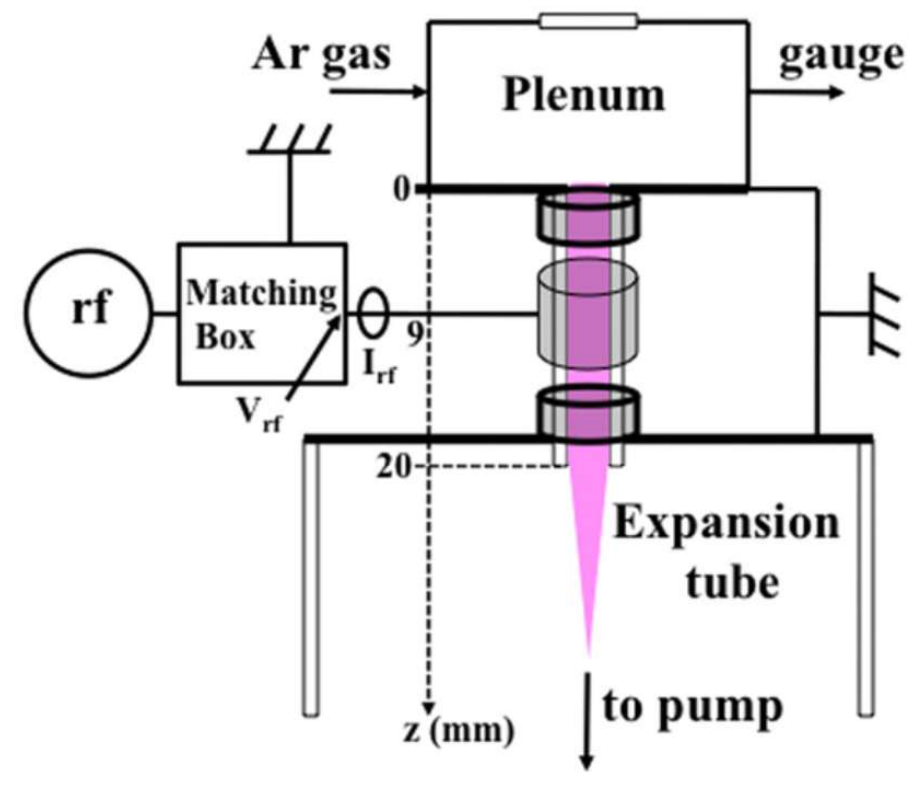

Figure 1.6: Pocket Rocket Device [34]

A number of papers have been published investigating the behavior, operating principles, and performance of this experimental thruster. One of the first experiments conducted was to image the radial plasma distribution from a window at the rear of the original device, which revealed that the PR has two operating modes: a discharge centered in the tube and an annular discharge against the wall. The centered discharge 
occurred for plenum pressures of less than 3 Torr, and the annular discharge was observed for pressures greater than this threshold [32]. This work was conducted with argon as the working gas, but similar results were achieved in a nitrogen discharge as well [33]. Further work has focused on the central discharge mode, as it has the attractive features of reducing heat transfer to the thruster walls and requiring less propellant mass flow [32] [34].

Following these initial probes into the behavior of the device, much more detailed work was done to understand the principles on which it operates. It has been determined both experimentally [34] and through validated CFD [5] that the capacitive discharge operates in the gamma mode, and is sustained largely by the acceleration of secondary electrons through the sheath around the RF electrode. Further CFD investigations revealed that the dominant mechanism by which the plasma discharge heats the neutral gas is through ion-neutral charge exchange collisions [30]. The neutral gas has also been experimentally shown to draw significant heat from the hot tube wall, as the transient temperature of the thruster plume correlates strongly with the predicted heating curve for the thruster body [5]. Efforts to improve the efficiency with which heat is transferred to the neutral gas should therefore focus on increasing the plasma density per unit power applied to the discharge or on reducing heat loss to the thruster structure through the thruster wall.

Thus far, efforts to increase the efficiency of the thruster have focused on tuning the operating parameters of the thruster, not the geometry of the device itself. These studies have confirmed the intuitive result that increasing the electrical power applied per unit mass flow, whether by increasing applied power or decreasing mass flow, increases the efficiency of the device [6]. This knowledge can be used to operate any given thruster configuration at higher efficiencies, but does not unlock the full potential of this device. To do this, effects of the geometry of the device itself must be explored. 


\subsection{Thesis Scope}

This thesis aims to determine the impact of RF electrode geometry on thruster performance by applying a mixture of experimental and numerical techniques. First, constant power tests of various thrusters with different electrode geometries will be run in the lab to provide experimental plasma density measurements at the thruster exit. Measurements from these tests will also provide the boundary conditions necessary to replicate each configuration in the CFD software package CFD-ACE+. Then, CFD simulations of each geometry will be run, and the simulated thruster exit plasma densities will be compared with the experimental values to validate the numerical model. The total numbers of ions and excited neutrals in the discharge tube from successfully validated models will be used to compare the performances of the different configurations. The best configuration will be the one to produce the highest degree of ionization in the discharge tube without generating excessive excited neutrals. These markers correlate with the most effective propellant heating and the least wasted energy, respectively. 
Chapter 2

\section{APPARATUS AND METHODOLOGY}

The apparatus for this study includes both physical and virtual components. First, the physical setup will be explored, followed by the diagnostics applied to this apparatus. Finally, the CFD model will be described in detail, including validation and verification efforts.

\subsection{Experimental Apparatus}

The physical apparatus for these experiments includes the fluid and electrical routing, as well as the design of the thruster itself.

\subsubsection{Testing Chamber and Plumbing}

Testing took place in Cal Poly's "Big Green" vacuum chamber, which is a large stainless steel bell-type chamber. The bell can be lifted with a hoist integrated into the chamber frame to allow access to experiments inside. The chamber is evacuated using a large rotary-vane roughing pump and monitored with two convectron-type vacuum gages. One gage is located on the KF-flanged pipe that connects to the pump, and the other monitors the inside of the chamber itself. The chamber includes many feedthrough ports and one seven inch diameter viewing window.

For these experiments, two 1/4 inch Swagelok tubing feedthroughs were used. One connected a convectron gage to the plenum of the thruster, and the other was used to supply the thruster with argon fuel. The argon was provided out of a standard DOT gas bottle, which was connected to the chamber through a regulator and manual valve. Argon flow into the thruster was adjusted manually using a combination of the 


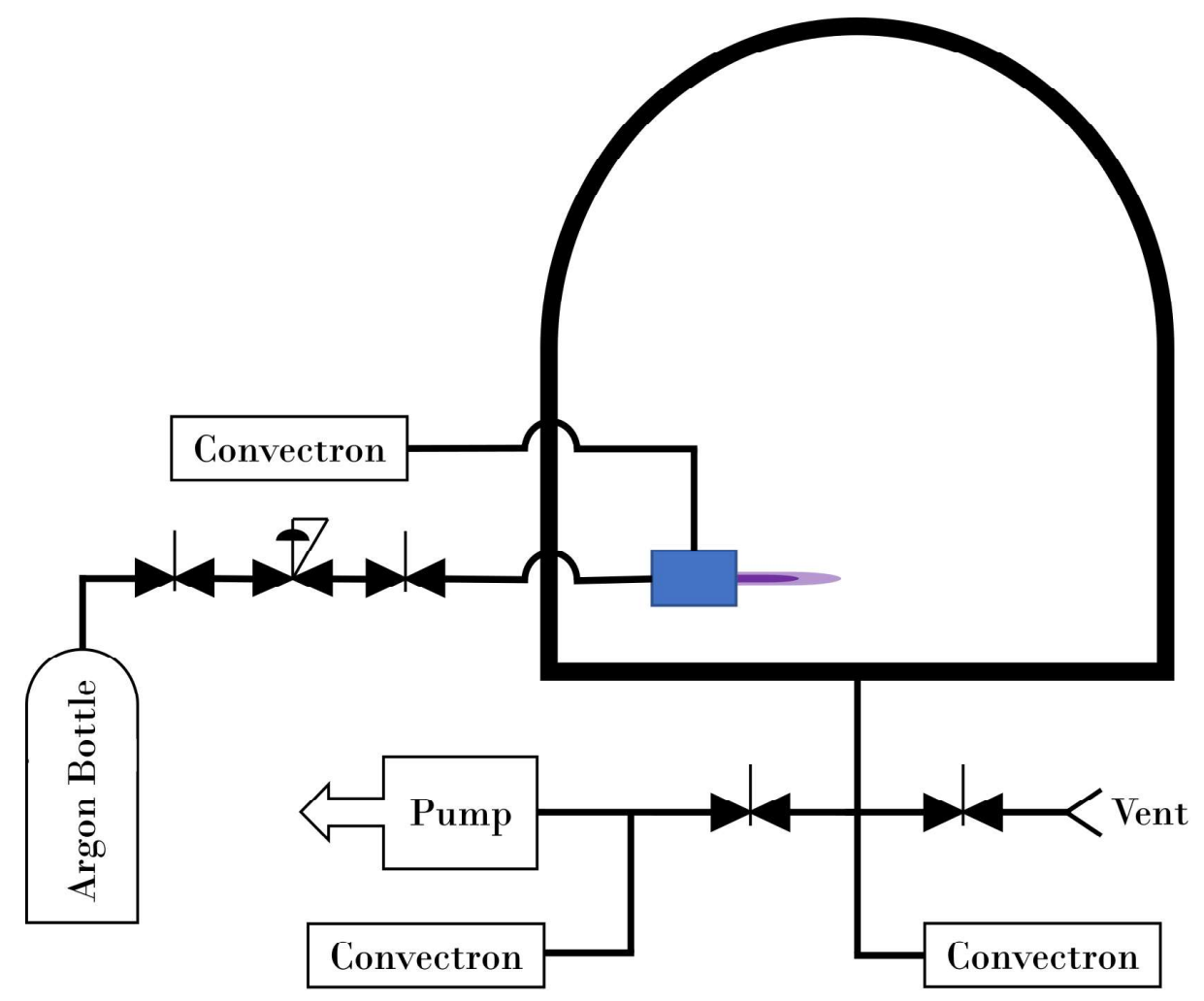

Figure 2.1: Plumbing Layout

regulator and manual valve, since the regulator was not built to maintain accurate pressures on the order of a Torr. The gage monitoring the thruster's plenum pressure was used to set the pressure as close to 1.5 Torr as possible during tests for consistency with past experiments.

The plumbing connections inside the chamber were made by adapting the Swagelok tube to hose barbs, then connecting silicone tubing to hose barbs on the thruster body. These hose barbs used standard UNC 10-24 threads and o-ring seals. The silicone tubing used was chosen under the naive assumption that silicones are low-outgassing materials. In reality, the outgassing performance of silicone varies greatly depending on polymer type, catalyst type, and bakeout procedure, meaning that low-outgassing varieties require special formulation [35] [36]. Fortunately, these tests did not require sufficiently low chamber pressures for possible outgassing of the silicone to become 
an issue, as the chamber pressure typically sat between 0.2 and 0.4 Torr.

\subsubsection{Electrical Routing}

The electrical components of this apparatus were configured to both power and measure the thruster. Power was supplied to the system by a Seren R301 power supply set to deliver $10 \mathrm{~W}$ at the ISM frequency of $13.56 \mathrm{MHz}$. To match the characteristic impedance of the system to the $50 \Omega$ of the supply, this power was fed into a Seren MM300 matching network, which uses an L-type design. The matching network was set at the beginning of each set of experiments such that the power supply displayed $0 \mathrm{~W}$ reflected power. Reflected power remained at zero for all further geometries tested, so changing the width of the electrode does not seem to significantly alter the characteristic impedance of the device.

From the matching network, RF power was routed straight through a T-junction into the vacuum chamber. The T-junction was used to connect an oscilloscope to the power line by way of a high impedance 1000X probe for measurement of the system's operating voltage. The oscilloscope measured peak-to-peak voltage and displayed a rolling average of 16 cycles. This measurement was necessary to later re-create lab tests in CFD. All power line connections outside the chamber were made with N-type hardware and $50 \Omega$ co-ax cable. A simplified schematic of this setup is shown in Figure 2.2.

Inside the chamber, power was routed from the feedthrough to the thruster using a $50 \Omega$ BNC cable, then a BNC to SMA adapter to interface with the SMA connection on the thruster. Also in the chamber was the connection made to the Langmuir probe, which was made using a single Teflon-insulated wire. This connected to a BNC feedthrough on the chamber wall, and proceeded to the Langmuir probe measurement circuit through another BNC cable. The layout of this circuit will be discussed in 


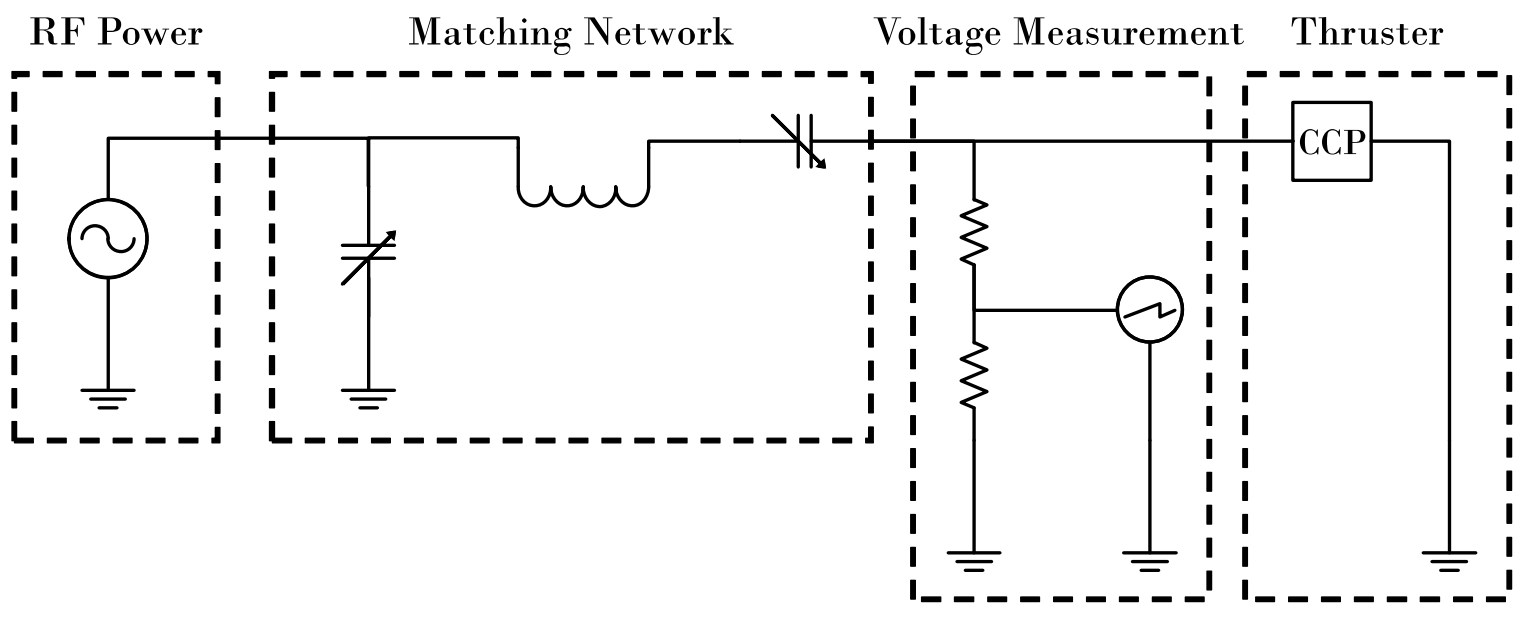

Figure 2.2: Simplified Power System Schematic

detail in Section 2.2.1.

\subsubsection{Thruster Hardware}

To enable testing of various electrode geometries with minimal effort and expense, a new thruster geometry was developed for this investigation. Instead of the solid copper ring typically used as an electrode in PR devices, copper tape was wrapped around the central alumina tube and connected to the RF power supply via a Tefloninsulated feedthrough. This design allowed the electrode to be easily cut to the desired size and to be repeatedly adjusted. To prevent stray discharge between the RF electrode and the ground electrodes, Kapton tape was wrapped around the alumina tube on either side of the RF electrode. This step may have been unnecessary, as plasma density measurements at the thruster exit appeared to show no statistically significant difference between tests run with and without the Kapton tape. This setup is shown in Figure 2.3.

The rest of the thruster body, shown in Figure 2.4, was built consistently with previous Pocket Rocket designs. The aluminum thruster body was created on a manual lathe, and finished on a manual mill. The plenum chamber at the rear of 


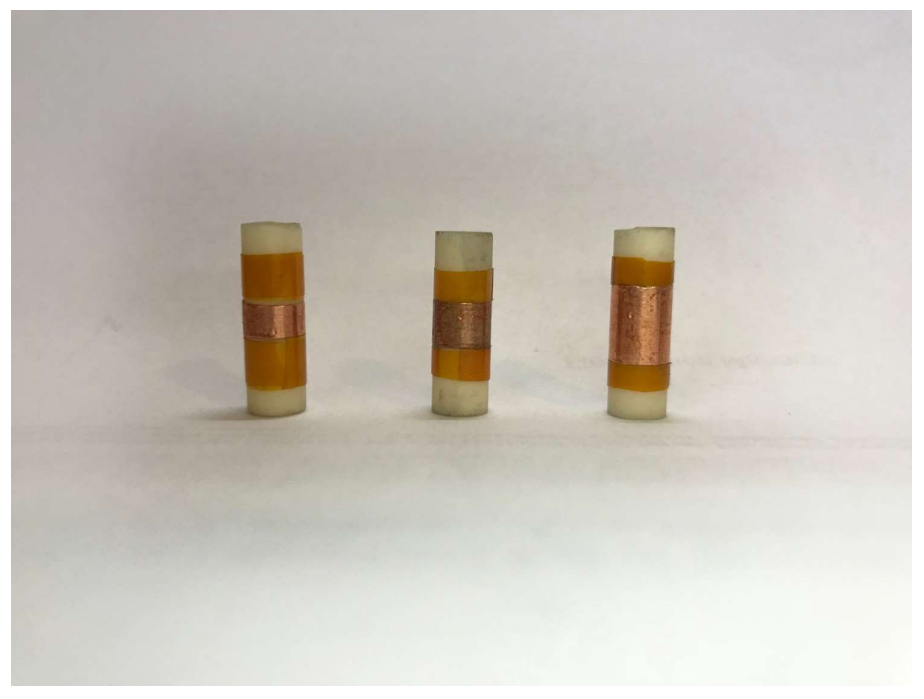

Figure 2.3: Selected Discharge Tubes

the thruster has two gas fittings attached - one to supply the propellant gas and one to measure the plenum pressure (not shown in the figure). A Macor cylinder was created to slide into the thruster housing to surround and insulate the RF electrode. A small hole through the side of this cylinder allows power to be fed to the electrode. A Teflon insert is press-fit into the thruster body to insulate the power feed from the grounded thruster. The feed continues to an SMA connector on the thruster surface that is attached to the power feed line. These internal components are held inside the thruster with an aluminum face plate, which also holds an O-ring in place to seal the thruster.

The dimensions of this thruster were accurately reproduced in CFD software and are shown in section 2.3. One important important dimension not in the CFD is the inner diameter of the Macor cylinder, which was drilled with a US standard G drill, and measures $6.4 \mathrm{~mm}$ in diameter. It is also important to note that the target electrode sizes were not manufactured precisely, so that, for example, the " $3 \mathrm{~mm}$ " electrode was not actually $3 \mathrm{~mm}$ wide. This was taken into account in the CFD re-creations of the experiments, which used the actual measured electrode sizes. The 


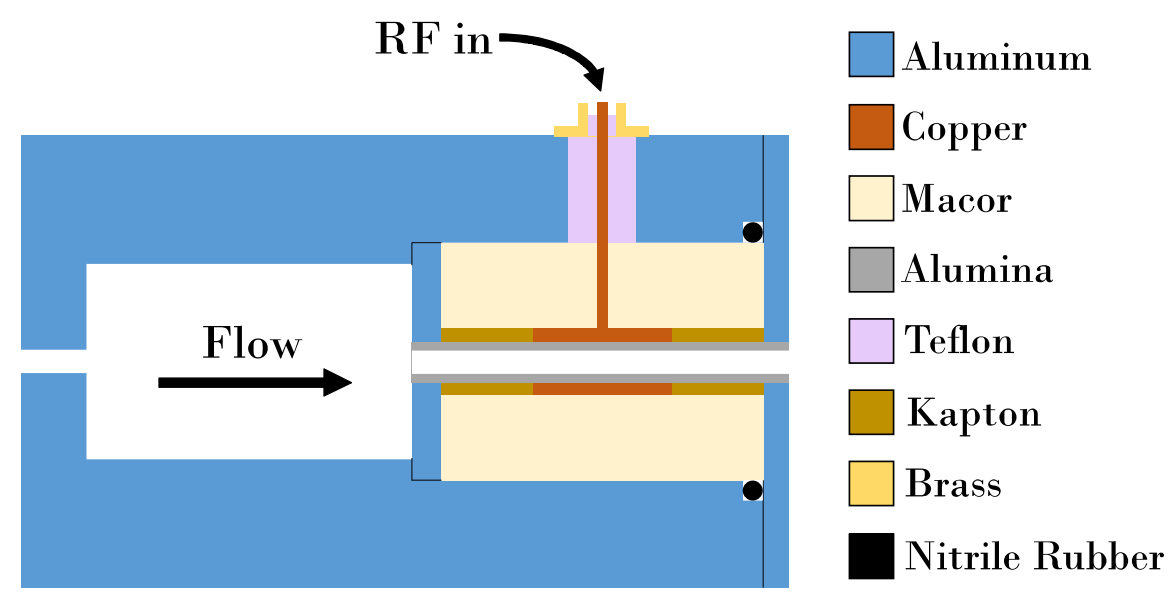

Figure 2.4: Thruster Diagram

relation between the targeted sizes and actual dimensions of each electrode is shown in Table 2.1. Electrodes are referred to by their target sizes for simplicity in this report. The measured values shown are an average of three measurements of different locations around the electrode circumference, and the error shown is the standard deviation of these measurements.

Table 2.1: Electrode Dimensions ( $\mathrm{mm})$

\begin{tabular}{|c|c|c|}
\hline Target Width & Measured Width & Measurement Error \\
\hline 2 & 2.69 & 0.1 \\
\hline 3 & 3.60 & 0.3 \\
\hline 5 & 4.93 & 0.1 \\
\hline 6 & 6.36 & 0.2 \\
\hline 8 & 8.58 & 0.1 \\
\hline 10 & 10.41 & 0.2 \\
\hline
\end{tabular}

To ensure consistent plume measurements between geometries, a scaffold was created to rigidly attach the Langmuir probe to the thruster body. Using the scaffold, the thruster could be disassembled and reassembled with a new electrode, and then measured in the exact same location. The scaffold consisted of an aluminum baseplate 
that was screwed onto the base of the thruster body with two threaded rods rising from the corners on the other end. These threaded rods held another pair of threaded rods spanning across the face of the thruster by way of two aluminum blocks. Finally, the Langmuir probe was held in place in a third aluminum block that rested on these horizontal rods using a set screw. Nuts threaded onto the rods allowed the probe to precisely shifted vertically and horizontally, then locked in place with respect to the thruster. The consisted of a wire approximately $0.2 \mathrm{~mm}$ in diameter protruding about $1 \mathrm{~mm}$ out of the end of a ceramic rod. The rod was ground down to a diameter at its tip of $1.2 \mathrm{~mm}$, which resulted in a cross-sectional area of less than $10 \%$ of the thruster tube. Finally, the hole through which the wire protruded from the ceramic was sealed with ceramic cement. This apparatus is shown in its entirety in Fig. 2.5 below.

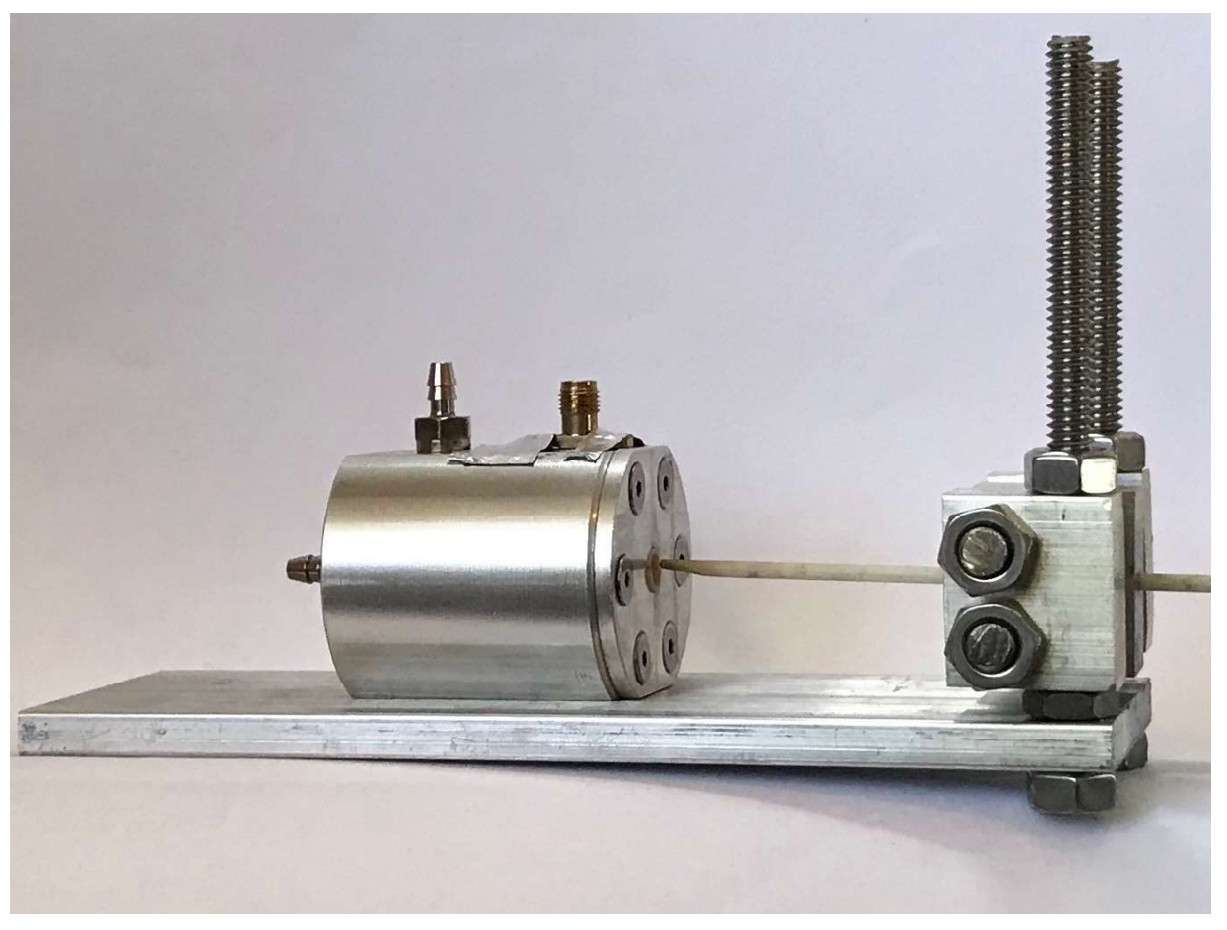

Figure 2.5: Experimental Apparatus 


\subsection{Data Collection}

Data was collected with both Langmuir probe measurements and an optical method. The Langmuir probe measurements provide quantitative data on the plasma density, while the optical results serve to increase confidence in the trends seen in the Langmuir probe data.

\subsubsection{Langmuir Probe}

Langmuir probes in their simplest form consist of a bare metal electrode exposed to the plasma which is biased with a DC voltage, causing it to exchange charge with the plasma bulk. Sweeping through a range of bias voltages and measuring the rate of this charge exchange generates a current-voltage (I-V) characteristic, which can then be analyzed using an appropriate technique [37]. Measuring an RF plasma introduces complexities, as a Langmuir probe in its simplest form will pick up RF oscillations in the electric field that obscure the desired I-V characteristic. Many techniques have been developed to compensate for this [38] [39] [40], typically involving using resonant LC filters to block the RF frequency and its harmonics. This is sometimes used in combination with a floating electrode connected to the main probe via a small capacitor, which helps the electrode voltage follow the RF variation of the plasma potential [38]. The effect of the filter network is maximized by locating it as closely to the probe tip as possible to minimize capacitance between the probe and ground. It is also possible, though more difficult, to directly bias the probe with an RF power source synchronized to the one driving the plasma [40]. These compensation techniques allow for the measurement of sensitive results from the I-V characteristic, such as the Electron Energy Distribution Function (EEDF). More robust features of

the I-V characteristic remain relatively unchanged even with moderate amounts of RF signal, though, so a simpler compensation scheme was deemed sufficient for these 


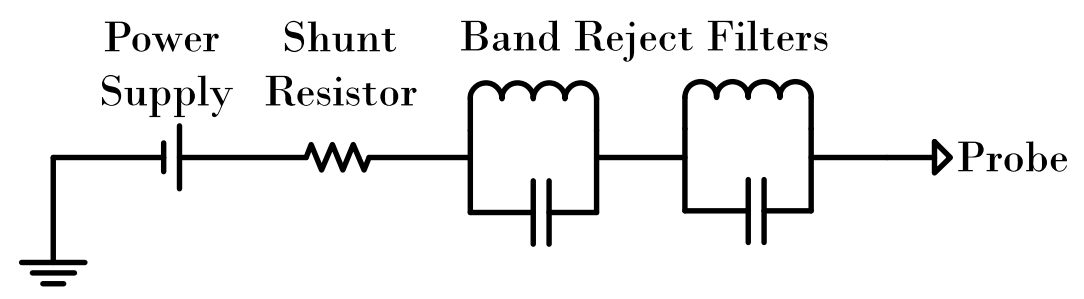

Figure 2.6: Langmuir Probe Electrical Schematic

experiments, similar to that described in [39].

As can be seen in Figure 2.6, RF chokes and capacitors were used to create two series band reject filters to block the RF signal from appearing in the probe data. The DC current from the probe passes these filters unimpeded (their resistance is much less than $1 \Omega$ ), and creates a measurable voltage drop across the $10 \mathrm{k} \Omega$ shunt resistor. This voltage drop is used to deduce the current collected by the probe at any given voltage. The reference connection for a digital oscilloscope (not shown) was connected between the power supply and shunt resistor so that the voltage across each could be independently measured. It is important for anyone wishing to duplicate this setup to note that most oscilloscopes internally connect the reference voltage input to ground, so the experimenter must make an effort to keep the oscilloscope ungrounded, otherwise the power supply output will be shorted to ground through the oscilloscope. The oscilloscope can be kept floating using an isolation transformer or by physically interrupting the connection that normally exists between the oscilloscope and the building ground. This configuration was chosen despite its complications because it allowed for the finest possible measurements of the probe current while keeping the power supply grounded.

In a typical test, six I-V traces were recorded for each thruster geometry, along with the plenum pressure, vacuum chamber pressure, and RF electrode voltage. The thruster was turned off and the chamber allowed to pump down after every other recording. Each pair of recordings consisted of one sweep from positive to negative 
voltage and another in the other direction in an effort to account for any lag present in the I-V trace due to capacitance in the probe circuit, though no such lag was directly observed. Each geometry was tested on at least two separate occasions, though some were tested more in an attempt to understand apparent anomalies that appeared during testing. These tests and anomalies will be discussed further in Chapter 3.

Analysis of the I-V characteristics focused on the more robust observable features, like the plasma potential, electron current exponential region, and ion saturation current [41]. This also allowed for the use of simpler methods to interpret these parts of the I-V curve. The first step in parsing probe plots was to apply a linear fit to the ion saturation region, then to subtract this from the measured data to produce the electron current [41] [42]. Other papers have shown $I^{4 / 3} \propto V$ [43] and $I^{2} \propto V$ [44] fits to work as well, but a linear fit was chosen for this work as it most closely matched the data. A typical example of a linear fit to this data set is shown by the orange line in Figure 2.7.

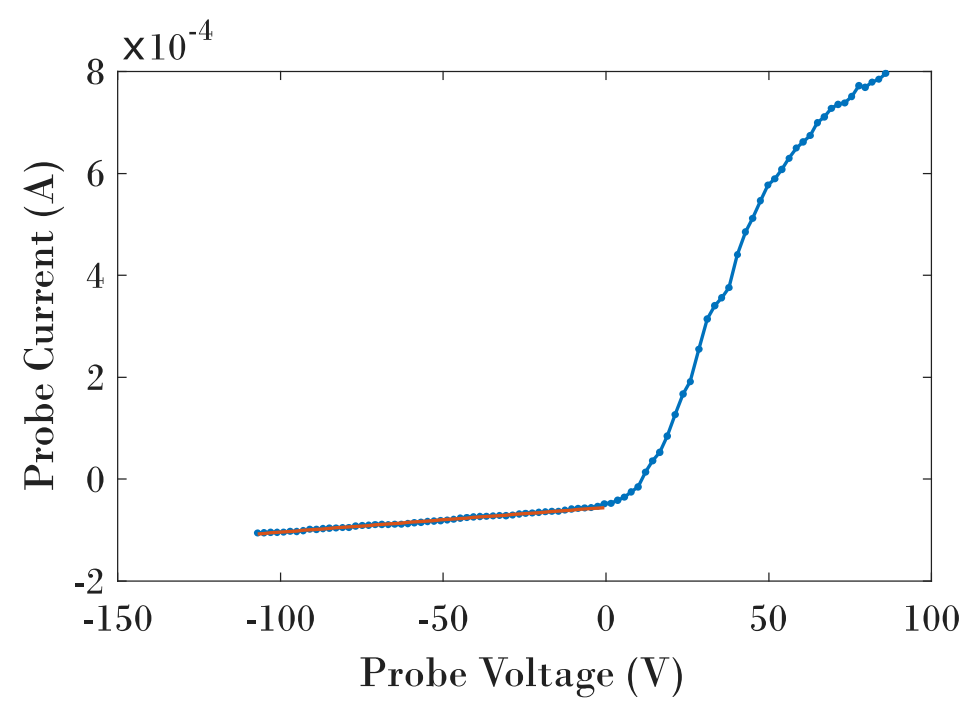

Figure 2.7: Linear Fit to Ion Current - Typical Example

Another linear fit was then applied to the natural $\log$ of this electron current plotted against voltage, as the slope of this line is the inverse of the electron temper- 
ature in electron volts. [41] [42] [43]. This region of the probe trace was occasionally problematic, so I-V characteristics that did not display linearity in these plots were discarded. An example of a typical I-V characteristic showing linearity and an example of a discarded data set are shown in Figure 2.8.

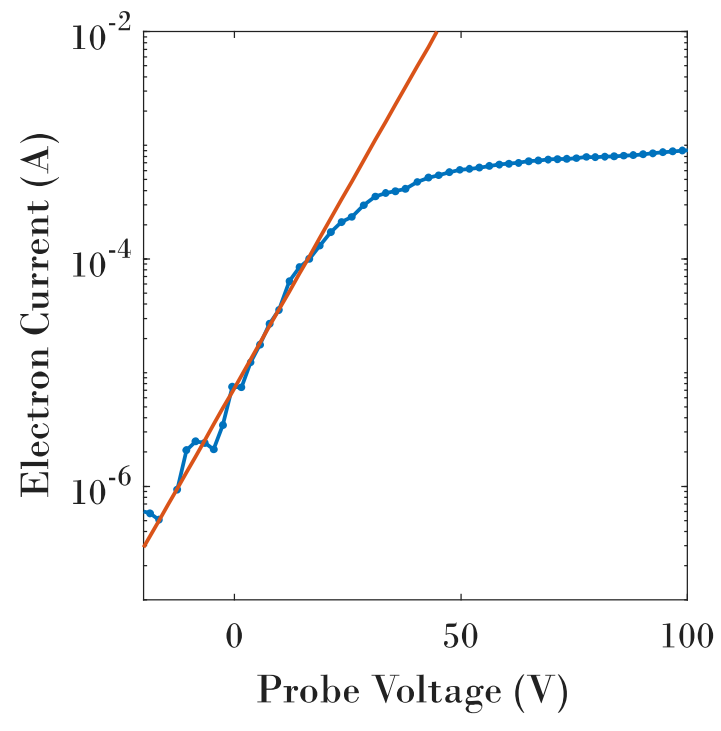

(a) Typical Fit

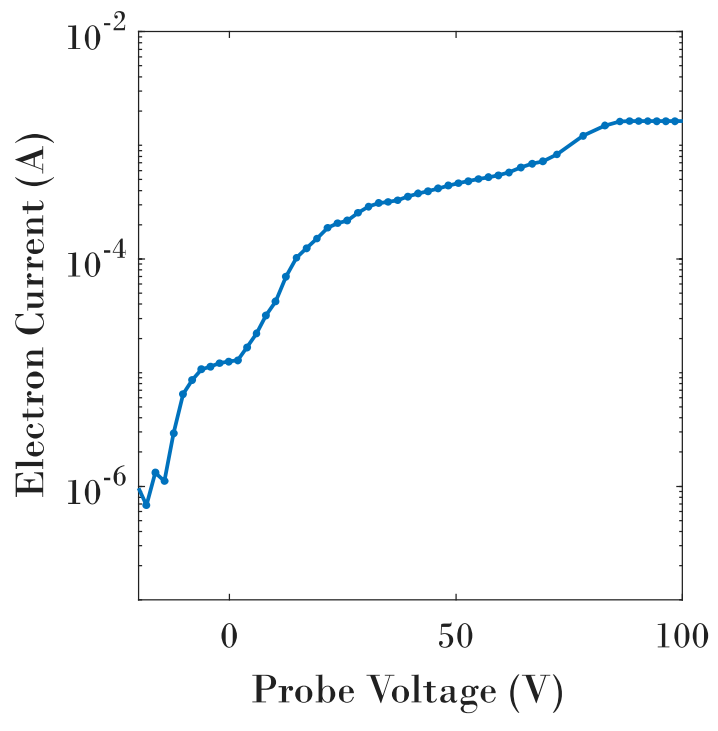

(b) Poorly Behaved Exponential Region

Figure 2.8: Exponential Fits to Determine Electron Temperature

Finally, the ion current was extrapolated forward to the floating potential using the linear fit created earlier, at which point a modified equation describing the Bohm criterion could be applied as shown [43].

$$
n=\frac{I_{i}}{\alpha A_{p} \sqrt{K T_{e} / M}}
$$

Here, $n$ is the plasma density, $I_{i}$ is the ion current, $T_{e}$ is the measured electron temperature, and the quantity $\alpha A_{p}$ replaces $\alpha_{0} A_{s}$, with $A_{p}$ being the area of the probe and $A_{s}$ being the area of the sheath. The modified $\alpha$ value used to make this replacement was generated from a paper by F. F. Chen, in which Langmuir probe data were scaled to match plasma densities measured with microwave interferometry [43]. This empirical method was used instead of more traditional Allen-Boyd-Reynolds 
(ABR) or Bernstein-Rabinowitz-Laframboise (BRL) methods, as these theoretically derived methods have been shown to give poor results for plasmas in the range of densities at which Pocket Rocket operates [44].

\subsubsection{Optical Diagnostics}

However, significant error is associated with this type of Langmuir probe data, so a second measurement technique was necessary to have confidence in the trends these first measurements seemed to suggest. Due to its simplicity and robustness, an optical measurement technique was chosen to fill this need that required only a camera, tripod, and bandpass filter. This data was taken concurrently with a set of Langmuir probe data to ensure the validity of the correlation.

Excited argon ions have a spectral emission band at 488nm, a wavelength which is visible to the naked eye and is not close to any spectral lines generated by neutral argon [45]. This makes it ideal to sample selectively to determine the quantity of excited argon ions in the discharge. To do this, a 488nm bandpass filter with a bandwidth of $10 \mathrm{~nm}$ was attached to a digital camera. By manually controlling all parameters of the shot, like the aperture, ISO, and shutter speed, consistent images could be taken of the plumes generated by each thruster geometry. Examples of the plume's appearance with and without the filter are shown in Figure 2.9.

The brightness of this image at the thruster exit then correlates to number of excited argon ions present, which is proportional to the total number of electrons squared [34]. Unfortunately, the tripod mounting setup used to collect these photographs moved slightly between each test, so pixels could not reliably be sampled from the same location in every shot. To remedy this, the centerline of each plume image was detected by averaging the locations of the highest and lowest pixels illuminated above a certain threshold. A swath of the image 11 pixels tall along this 


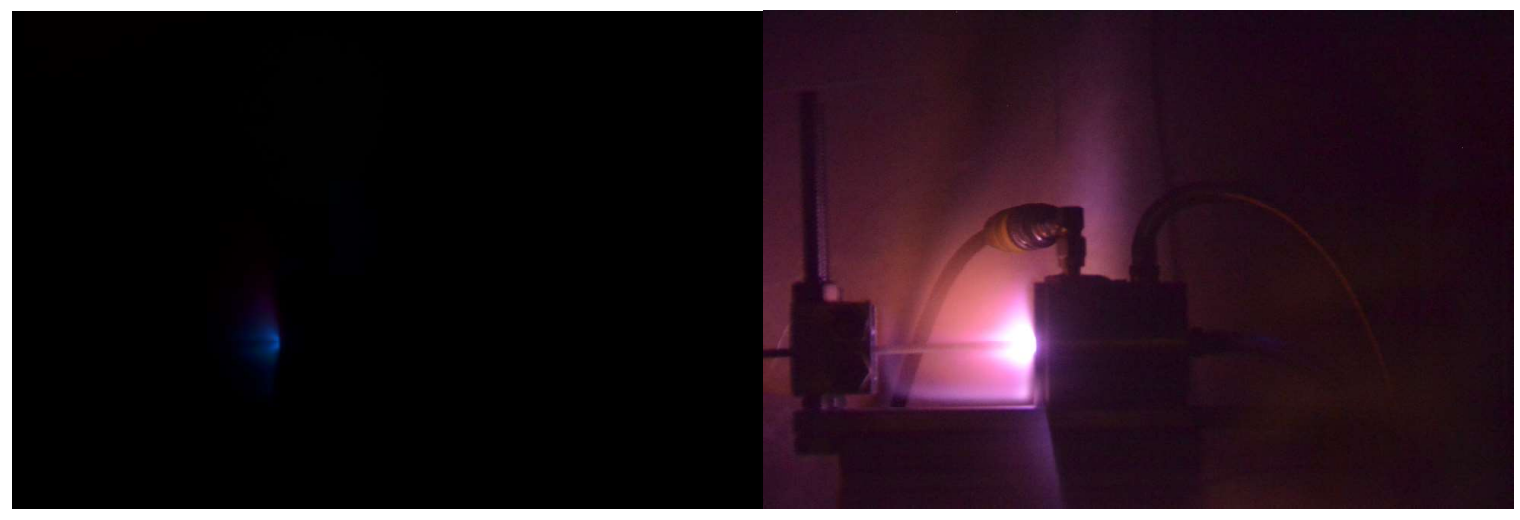

(a) Plume Appearance with 488nm Filter

(b) Plume Appearance Without Filter

\section{Figure 2.9: Plume Photographs}

centerline was then sampled from each image. The blue channels of the brightest 100 pixels in these samples were averaged to represent the intensity of the light measured in the plume. Because this intensity is proportional to the number of electrons squared, the square root of the measured intensity was used to compare with Langmuir probe data. Unfortunately, since an argon plasma of known density was not available, these images could not be calibrated to provide quantitative comparison against the Langmuir probe measurements. Instead, the trends observed by both methods were compared to give additional confidence to the trends observed with the Langmuir probe.

\subsection{Computational Fluid Dynamics Model}

Though the experimental diagnostics described above proved to be very capable of directly determining properties of the plasma generated by the thruster, it is woefully impractical to experimentally measure the flow field at enough locations to build a complete picture of the discharge. Using CFD, however, any property in the flow field can be extracted at any time and location. This can be an extremely powerful tool in understanding the behavior of the thruster, but only if the simulated results mimic 
what actually occurs in the lab. For this reason, it is important to both verify and validate a CFD model. In the context of CFD, "verification" refers to the process of ensuring that a well behaved, self-consistent model has been created, and includes activities such as grid convergence and boundary convergence studies. "Validation" refers to the act of comparing CFD results against experimental data to ensure that the model accurately represents the situation of interest. Model creation and verification will be discussed here, as well as validation against previously published results. Validation against the experimental results collected as a part of this study is covered in Chapter 3.

\subsubsection{CFD Model Description}

This model was created in the software package CFD-ACE+, which is one of the few commercially available CFD packages capable of simulating plasma discharges with enough detail to capture the behavior inside the Pocket Rocket [5] [30]. The domain created for these simulations is $2 \mathrm{D}$ and axisymmetric, and includes, from left to right, the thruster's plenum, discharge tube, and a volume aft of the thruster for the plume to expand, as shown in Figure 2.10. The envelope dimensions of the domain are shown on the figure as well.

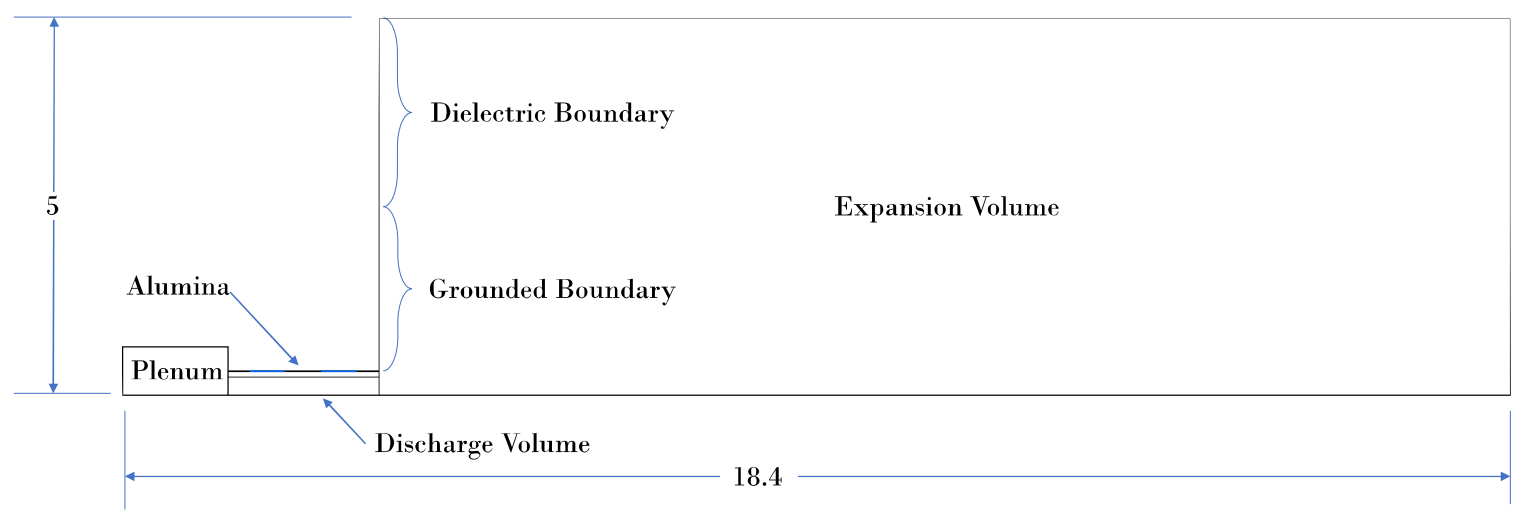

Figure 2.10: Full Simulation Domain 
At the far left and right boundaries of the model are constant pressure inlet and outlet boundary conditions to replicate the pressures measured experimentally in these areas. The lower boundary is the axis of symmetry. Along the top surface of the alumina wall of the discharge tube, the domain boundary is divided into five segments: a ground electrode on either end, the RF electrode in the center, and dielectric boundaries in between. The edge of the domain at the aft end of the thruster is split in half; the lower half is a grounded, conductive wall to mimic the thruster housing, and the upper half is dielectric. When attempting to replicate experimental measurements, the two pressure boundaries and the RF electrode voltage were set to the average measured values for the validation case in question.

The dimensions of this domain were sized to exactly match the dimensions of the physical thruster used in this study. Figure 2.11 shows all of the dimensions used. Also highlighted are the various boundary conditions applied to the outside of the alumina tube surface. All dimensions in the domain remained constant between all simulations with the exception of the RF and Dielectric widths. As the RF electrode changed in size, the widths of the Dielectric conditions were changed correspondingly to maintain the $20 \mathrm{~mm}$ overall length of the discharge tube.

This domain is filled with a structured mesh, shown in Figure 2.12. Mesh density was determined by using the lowest possible number of cells in the discharge tube, which is the most sensitive area of the simulation, and then expanding the mesh as much as possible while avoiding highly skewed cells. An unstructured mesh would have been more appropriate for the expansion section of the model, as it would have allowed the cell density to be reduced much further while also reducing the skewness of the mesh. Unfortunately, CFD-ACE+ does not yet allow combining structured and unstructured domains into a single simulation, so a structured grid was used everywhere. Introduction of some higher aspect ratio cells was unavoidable to keep the simulation cell count manageable, but the most highly skewed cells occur in areas 




\section{Figure 2.11: Thruster Domain Closeup With Dimensions (mm)}

of little to no flow velocity, and so are not a major concern.



Figure 2.12: CFD Mesh

A zoomed in view of the discharge tube mesh is shown in Figure 2.13. This shows the extremely low aspect ratio of the cells throughout this important region of the domain. Also of note is the lack of cells concentrated against the tube wall that are typically included to capture boundary layer dynamics. At the extremely low Reynolds numbers experienced inside the thruster, there is less than a $3 \%$ difference between laminar and turbulent simulations [5], so the difference between simulated 


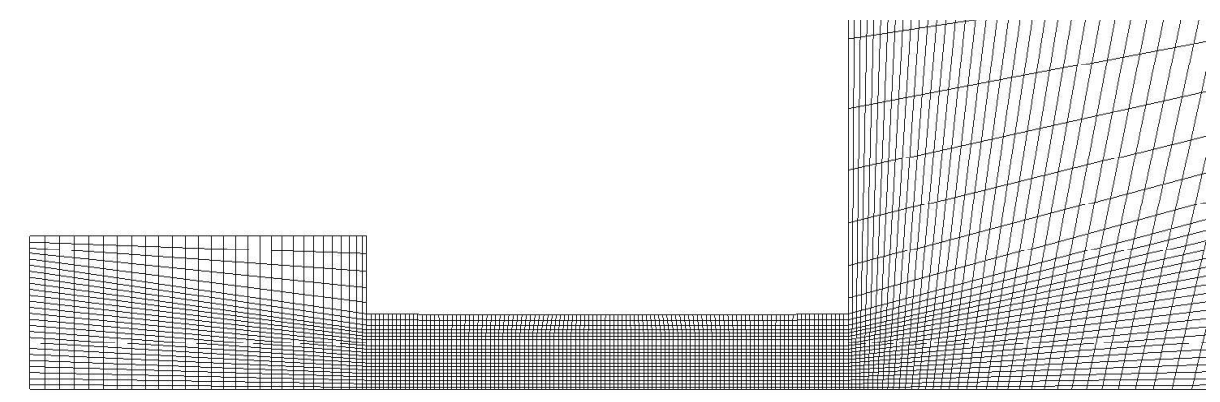

Figure 2.13: Discharge Tube Mesh

and modeled boundary layers should be even less. This omission was therefore deemed acceptable, and speeds up the simulation significantly.

To simulate this domain, the flow, turbulence, heat transfer, chemistry, electric, and plasma packages were all used in a transient simulation to reproduce these conditions. Full details of the solver mechanics can be found in the CFD-ACE+ user manual [46], but a summary is presented here. The flowing argon was modeled using a RANS method with the ideal gas law and solved with a SIMPLEC-based algorithm. A $\mathrm{k}-\epsilon$ turbulence model was used to track boundary layer growth against the tube walls. The chemistry module was used to track the concentrations of various species in the discharge; specifically argon neutrals, argon ions, excited argon atoms, and electrons. This module also simulates interactions between species, specifically the ionization, stepwise ionization, excitation, and neutralization processes by tracking the concentrations of each species in each cell and applying the appropriate reaction rates calculated from JILA cross-section data. The reactions modeled are ionization, excitation, and stepwise ionization. For these last two reactions, the exact energy level of the excited state is not tracked, so all possible excited states are simplified into a single species. The concentrations from these calculations are then fed to the plasma and electric modules to calculate the plasma interactions between charged particles and the electric fields in the domain. The electric module calculates the electrostatic potential everywhere in the domain by combining the fields from all voltage sources 
using Gauss' law and the appropriate loss coefficients for each medium. The plasma module combines Maxwell's equations, energy balance, and an analytic sheath model as described in [47]. Because the electrons in the discharge move much faster than ions or neutrals, two distinct timesteps are used for the simulation. Electric fields and electrons are simulated with a timestep 20 times faster than that of the larger particles. However, some physical effects were still neglected, most notably the heating effects that the plasma has on the neutral gas. Though several attempts were made to use the tools built into CFD-ACE + to include this additional fidelity, they all resulted in problematic nonphysical behavior, and so were not used in this study. Despite this omission, though, the CFD model appears to accurately represent the plasma parameters of the physical system, as will be shown in Chapter 3. Similar models were applied successfully in other Pocket Rocket studies [5] [30]. An exhaustive list of model settings can be found in Appendix C.

To run the CFD models, a Python macro was generated to allow for a sequence of solver parameters to be stepped through automatically. The final sequence for this study began by running 100 timesteps at 30 iterations each to allow the simulation to converge from the coarse initialization parameters to a rough solution. The relatively high number of iterations per timestep allowed the simulations to converge towards a meaningful solution in fewer timesteps, and also enhanced the stability of the simulation, since these first hundred timesteps were the most common time for simulations to diverge. Then, 2900 timesteps were run at 8 iterations per timestep to help the simulation move quickly past startup transients. Plasma collision frequency was also changed from a constant value to be a function of electron temperature at the beginning of this stage. After this, 1000 iterations were run at 12 iterations each to somewhat improve the convergence of each timestep. Finally, a finishing pass of 60 timesteps at 30 iterations each was run for a better-converged solution. This sequence was determined by trial and error to be a good balance between solver speed, 
stability, and precision, and took approximately three hours to run per simulation at the final mesh size. For a run of all six experimental geometries, this amounted to 18 hours of runtime. These batch runs were also automated via a python macro which can be found in Appendix B.

\subsubsection{Model Verification}

This CFD model was verified by conducting convergence studies on both the mesh density and the outlet location. A timestep convergence study was not conducted, as the same timestep was used as the validated CFD in Reference [5]. The mesh density study focused on the number of cells in the thruster's discharge tube, as this is by far the most sensitive area of the mesh. Increasingly finely meshed versions of the $5 \mathrm{~mm}$ electrode geometry were simulated using the same simulation parameters, after which electron temperatures at the discharge tube exit were sampled and compared. The electron temperature was selected as the metric for convergence because the plasma parameters are the most sensitive quantities in these numeric simulations. Two perspectives on this convergence study can be seen in Figure 2.14.

The left plot shows the convergence of $T_{e}$ as the number of cells in the discharge tube increases, and the right plot shows how mesh density impacted temporal trends in $T_{e}$ results. Based on these plots, the mesh with 1921 cells it the discharge tube was selected, as it falls nicely in line with the converged trend in the left plot, and closely tracks the much higher cell count simulation in the plot on the right.

The second convergence study was used to determine the distance that the outlet was required to be from the thruster exhaust. Four different meshes were tested for this purpose, the shortest of which placed the outlet only $5 \mathrm{~cm}$ away from the thruster. As can be seen in Figure 2.15, the wall position made surprisingly little difference, so the wall was placed to capture somewhat more than the plume length visible in 


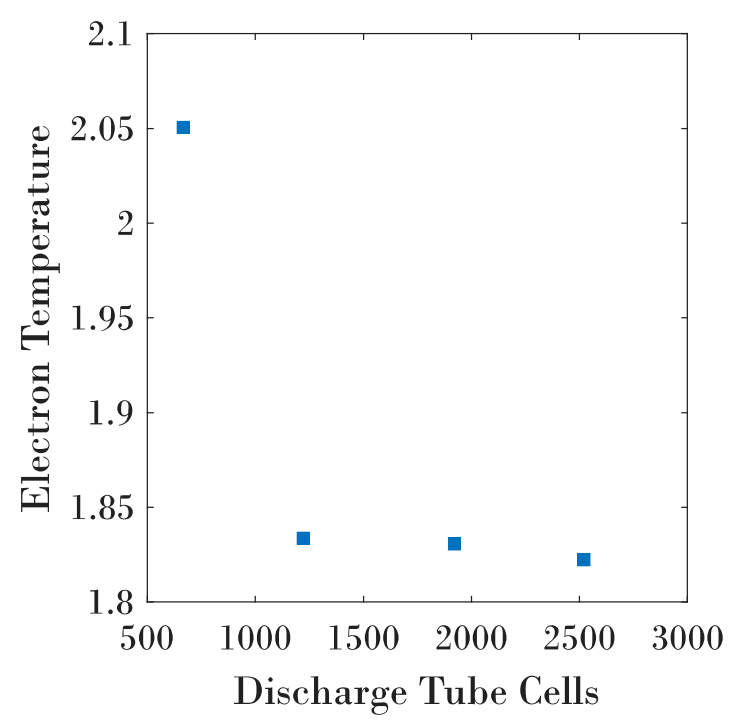

(a) Variation in Tube Exit $T_{e}$

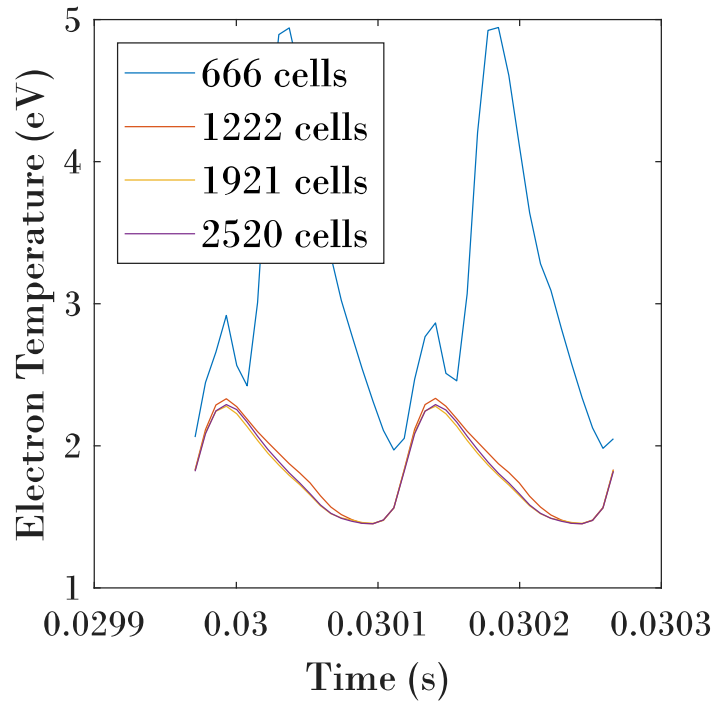

(b) Temporal Variations in $T_{e}$

Figure 2.14: Grid Convergence Results

experiments, $15 \mathrm{~cm}$ from the thruster's exit.

With these two model convergence exercises complete, the model had been verified and could proceed to validation.

\subsubsection{Preliminary Validation}

The first step in validating this model was to compare it against the existing body of literature. Most notably, work by Charles and Boswell showed that plasma density along the central axis of the discharge tube had a single central peak, trailing off to either side [34]. This same distribution has been replicated as shown in Fig. 2.16.

Also published in the same study were values of $\mathrm{T}_{e}$ and plasma density at a location $4 \mathrm{~mm}$ into the discharge tube in the downstream direction. This test used a plenum pressure of 1.5 Torr, the same value used in this CFD, but used an RF voltage of $380 \mathrm{~V}$ peak instead of the $198 \mathrm{~V}$ used here. This higher voltage, and therefore higher power throughput, should result in somewhat higher $\mathrm{T}_{e}$ and density values, which is 


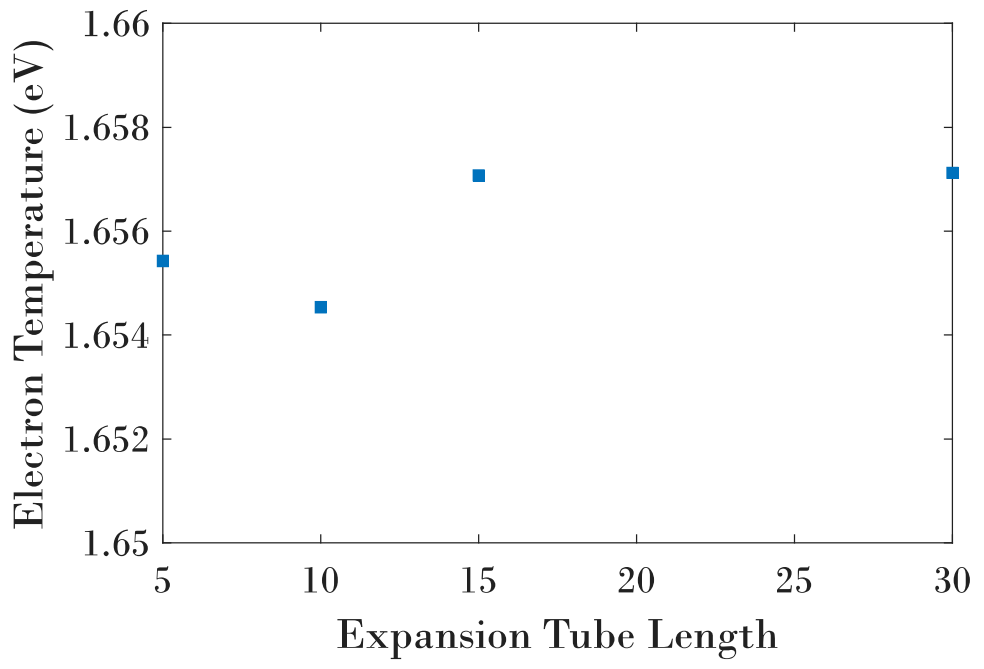

Figure 2.15: Wall Convergence

exactly what we see. The published $\mathrm{T}_{e}$ was $3 \pm 0.5[34]$, and the mean $\mathrm{T}_{e}$ in this location in CFD was 2.3. For plasma density, Charles et. al. measured $2.8 \times 10^{17} \mathrm{~m}^{-3}$, while this CFD model returned a phase averaged value of $2.56 \times 10^{17} \mathrm{~m}^{-3}$, or $91 \%$ of the published value [34]. Given the aforementioned difference in operating power, and the fact that the error on the published density measurements may be on the order of $50 \%$ [34], these comparisons agree quite well. Due to the shortcomings of this model with regards to thermal phenomena, published experimental measurements of parameters such as gas temperatures and thrust values could not be used for validation. However, additional validation data was created as a part of this study, and will be discussed in the next chapter. 




Figure 2.16: Plasma Density along Thruster Axis 
Chapter 3

RESULTS

The results of this study include both experimental and numerical investigation into how the behavior of a PR thruster changes as the width of its RF electrode is varied. First, experimental results will be presented, which consist of RF feed voltage measurements, Langmuir probe measurements of plasma density at the thruster outlet, and spatially resolved optical intensity measurements of the $488 \mathrm{~nm}$ argon ion emission band. These results will then be used to further confirm the validity of the numerical model described in Chapter 2. Finally, information about the interior of the discharge from this numerical model will be used to draw conclusions about the impact that RF electrode size may have on thruster performance.

\section{$3.1 \quad$ Voltage Measurement}

Since the power supply used in these experiments outputs a constant power, the voltage in the system depends on the impedance of the physical device. As discussed in Chapter 1, this impedance varies with electrode width due to the changing capacitance of the junction between the RF electrode and the plasma bulk. Due to loss within the power delivery system, the CFD could not be matched to experiments by applying the $10 \mathrm{~W}$ that leave the power supply to the simulated plasma, as this would be much more power than the experimental plasma absorbed. Measurement of the RF electrode voltage was necessary to complete these comparisons. A plot of these measurements against electrode width is shown in Figure 3.1, along with a linear trend line. This trend line, $V=-5.8151 W+223.64$, can be used by anyone wishing to duplicate the CFD results presented here without re-building the laboratory setup to collect 




Figure 3.1: Measured Electrode Voltage Vs Electrode Width

boundary condition information.

\subsection{Langmuir Probe Measurements}

In total, four rounds of Langmuir probe testing were conducted to ensure the reliability of these results and to compensate for anomalies observed during testing. Plasma densities produced from the first two rounds of testing are shown in Figure 3.2 with error bars of one standard deviation.

While some results appear to be nicely repeatable, such as the $3 \mathrm{~mm}$ and $6 \mathrm{~mm}$ cases, others (particularly the $10 \mathrm{~mm}$ case) are clearly not. In addition, the trends formed in each test differ; the first series of testing falls more or less on a straight line, while the second round of testing may show a correlation between electrode width and the plasma density at the thruster exit. However, one external factor may explain many of these discrepancies. Before the original tests, the thruster body was never fully cleaned of manufacturing residue, which could easily have vaporized inside the thruster plenum over the course of several tests. The alumina tubes used in what is 


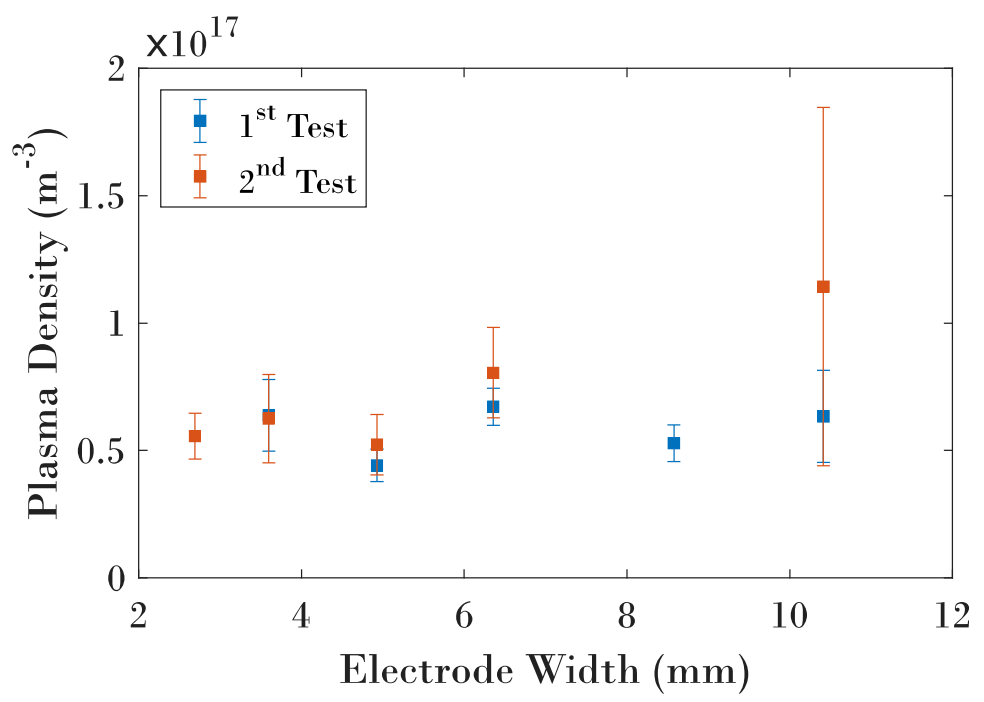

Figure 3.2: First Two Langmuir Data Sets: $1 \sigma$ Error Bars

here labeled as the first round of testing had been previously fired for many minutes as the thruster apparatus was tuned and tested. These tubes, then, would have been the most contaminated by manufacturing residue - particularly the tube used in the $5 \mathrm{~mm}$ test. This was the first tube fired, and was used in all further testing as an unaltered control, since the $5 \mathrm{~mm}$ electrode size has been used in all PR devices until now. The second round of tests, though, swapped back and forth between a new tube and the two old tubes. The first configuration run with the new tube was the 10 $\mathrm{mm}$ setup. Unfortunately, after this point, which tubes were used for which electrode sizes was not recorded, except that the same $5 \mathrm{~mm}$ tube was used as a control.

To investigate the possible impact of surface contamination inside the discharge tube, another round of testing was conducted where the inside surface of the discharge tube was swabbed with acetone and rinsed with isopropanol prior to each use. After the initial cleaning of the thruster tubes, particularly the $5 \mathrm{~mm}$ tube, marked discoloration was observed on the cleaning swab. Cleanings between subsequent configurations showed no such discoloration, but were conducted in the same manner as before to ensure consistency of the results. The one exception was the $3 \mathrm{~mm}$ configu- 


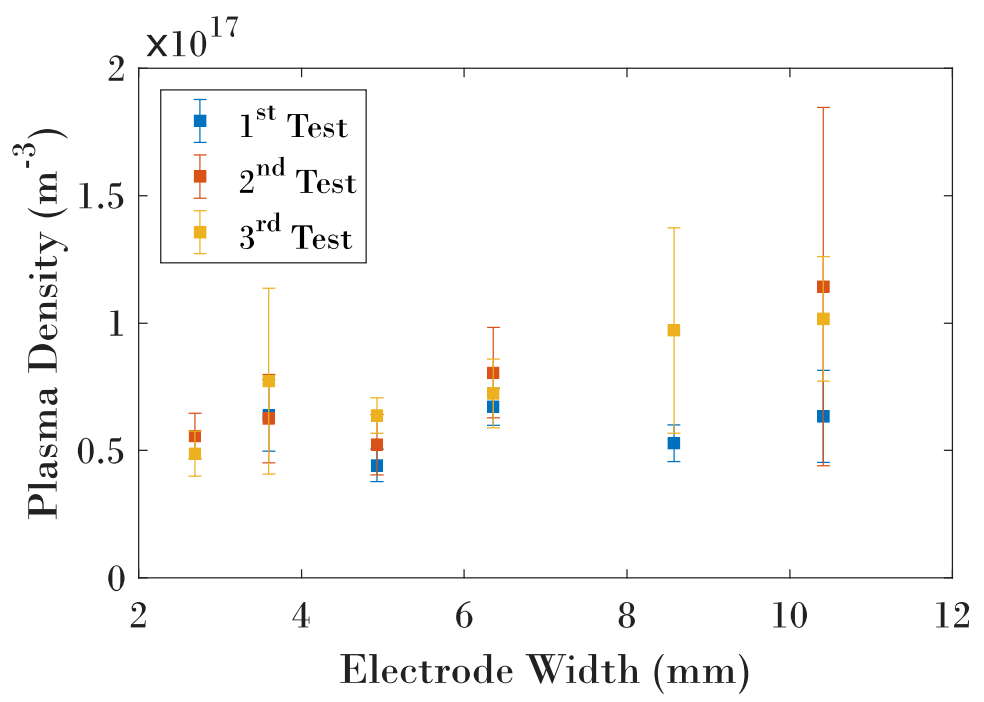

\section{Figure 3.3: First Three Langmuir Data Sets: $1 \sigma$ Error Bars}

ration, for which this cleaning step was accidentally omitted. The results of this third test are shown superimposed on the earlier results in Figure 3.3.

The most interesting features of this plot occur at the $10 \mathrm{~mm}, 5 \mathrm{~mm}$, and $3 \mathrm{~mm}$ electrode sizes. The $10 \mathrm{~mm}$ test appears to show alignment between the $10 \mathrm{~mm}$ test conducted with a factory finished tube and a manually cleaned one, and suggests that the contamination in the discharge tube had a significant effect at the large electrode sizes. The $5 \mathrm{~mm}$ test jumped up to the trendline formed by the other tests, whereas before it had sat clearly below the rest. This jump is smaller than the gap at the $10 \mathrm{~mm}$ end though, despite the $5 \mathrm{~mm}$ tube originally being much more heavily contaminated. The lone point that sits outside of the trend formed by the other configurations is the $3 \mathrm{~mm}$ case, which, as previously mentioned, did not receive the same cleaning treatment as the others. This does not necessarily invalidate the test, though, since no visible contamination was observed on the cleaning swab for the trials before or after this one. Another possible justification for this jump will be discussed in Section 3.5.

To build a convincing case for the trend uncovered by this third round of testing, a 




\section{Figure 3.4: Langmuir Probe Data with True Error Bars}

fourth and final series of tests was conducted. However, due to issues with the vacuum pump used for these tests, the back-pressure on this final round of testing was two to three times higher than in previous tests. For this reason, these measurements have not been added to the previous comparison, and were only used to compare against the optical measurement method.

The standard deviation error bars used thus far help convey the variance in the measured data, but due to the many sources of error in this Langmuir probe setup, these error bars are a misrepresentation of the total error involved. With more appropriate error bars, shown in Figure 3.4, the trends that seemed clear are much less conclusive. These error bars are a combination of the standard deviation and $25 \%$ of the measured value to capture experimental variance introduced by deviations in the probe tip size, shunt resistance, atmospheric humidity, probe surface conditions, and the error introduced by the Langmuir probe analysis method. To remedy this, optical measurements of plasma density were conducted in an attempt to corroborate these trends. 


\subsection{Optical Diagnostics}

As previously discussed, optical diagnostics were included due to improve confidence in the Langmuir probe data, despite the significant error inherent to the method. It appears this comparison was a success, as can be seen in the overlaid comparison of the optical and Langmuir probe results from the final round of testing shown in Figure 3.5 below.

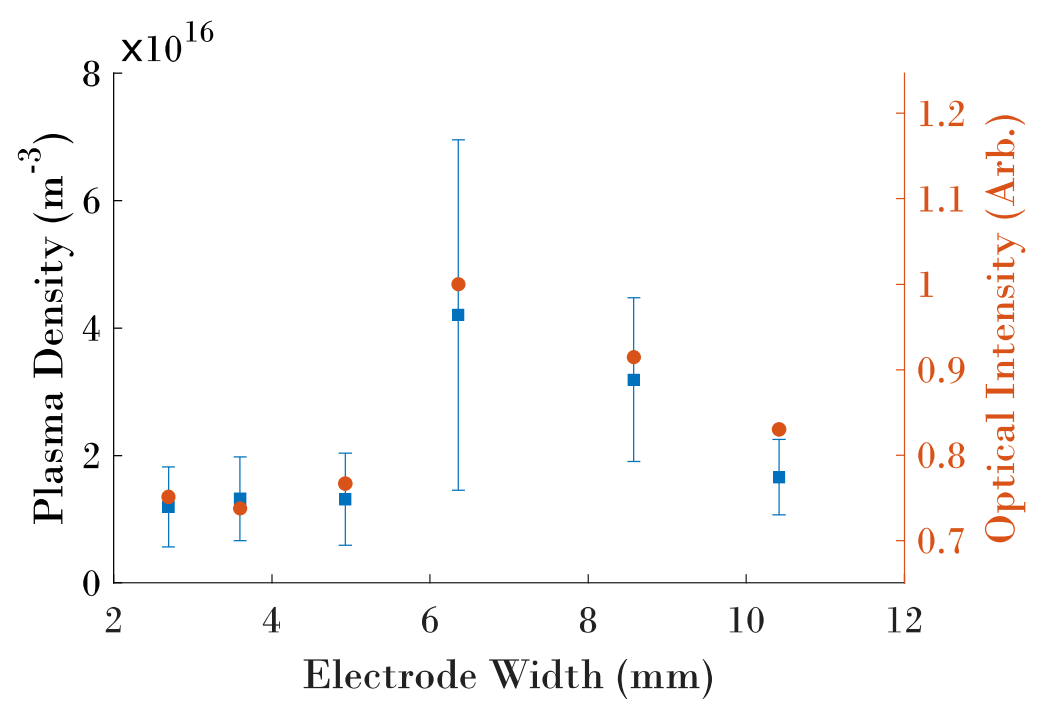

Figure 3.5: Optical Versus Langmuir Probe Results

Though individual points on the Langmuir probe plots may stray away from the true plasma density, the trends represented by these data appear to be correct. It is important to note, though, that the camera data is in arbitrary units, so it was shifted and scaled to produce the best match with the Langmuir probe data by the method of least squares. Also, the y axis for the optical intensity data does not start at zero, so the differences in intensity appear magnified. This could be because the plasma in the thruster plume is optically dense, so that the true intensity of the center of the plume is not visible from the outside. It may also have to do with nonlinearities present in the camera's sensor and image processing behavior. Lastly, some pixels in the 6 
mm configuration were saturated, so its true optical brightness may be somewhat higher than shown. However, given the otherwise excellent agreement between the two measurement methods, these are not significant concerns. The robustness of this comparison reinforces the conclusions made in the previous section based on the Langmuir probe data alone, and means that the next step may begin: validating the CFD model with this Langmuir probe data.

\subsection{CFD Validation}

With a wealth of data now collected on the behavior of the thruster with various electrode configurations, the CFD model could be verified across the range of geometries tested. A comparison of CFD data against the experimental data is shown in Figure 3.6 .

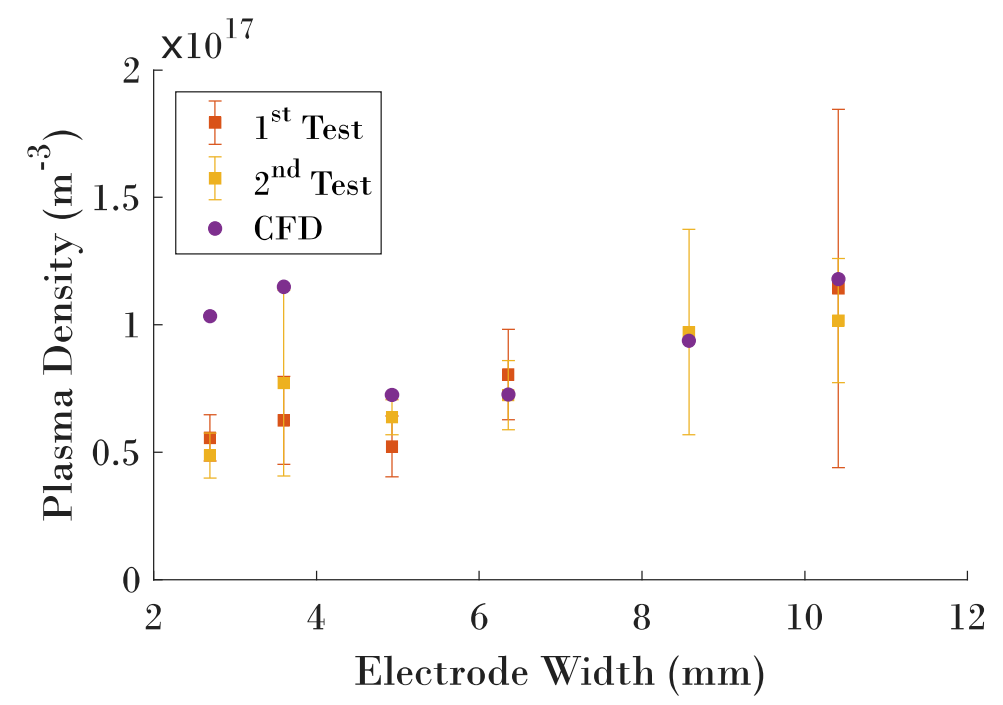

Figure 3.6: CFD Validation

As can be seen in the figure, good agreement is found for the larger electrode sizes (from $5 \mathrm{~mm}$ to $10 \mathrm{~mm}$ ), but agreement is less concrete for the narrower electrodes. Possible reasons for the poorer agreement at the $2 \mathrm{~mm}$ and $3 \mathrm{~mm}$ geometries will be 
explored in section 3.5. The CFD trend was generated by applying the experimentally measured boundary conditions for each configuration in Test 3 to the CFD boundaries and by applying a $\gamma$ value to the alumina wall determined by the RF electrode voltage. This relationship was applied because $\gamma$ values vary with incoming particle energy, and the energy of ions accelerated through the sheath will be directly proportional to the potential drop across the sheath, which itself varies directly with the RF electrode

voltage. The formula used to determine the $\gamma$ value applied is $\gamma=\frac{V_{R F}}{3116}$, which was found by selecting a $\gamma$ that happened to work for a single geometry and using it to define a line that passed through the origin. This function produced $\gamma$ values between 0.067 and 0.053 for the tested geometries; comfortably within the range of sensible values of 0.02 to 0.1 [27]. After expanding the range of $\gamma$ values used, the extreme ends of the range were also checked to ensure that they still produced the axial plasma density distribution measured by the ANU team, as discussed in Section 2.3.3.

\subsection{CFD Results}

With the CFD model now validated across the range of tested geometries, it could be used to examine various properties inside the thruster that likely correlate with performance. The most important of these parameters is the number of argon ions in the discharge tube, since collisions between these ions and the neutral atoms are responsible for the majority of the heating that the neutral gas experiences [30]. By revolving each cell around the axis of symmetry of the simulation, the volume represented by each cell could be obtained. Multiplying the ion density within that cell then gave the total number of ions inside the volume that the cell represented. Applying this to all cells in the discharge tube simultaneously allowed total ion quantities to be easily determined for each discharge geometry at a given timestep. Totals from the 20 timesteps that cover a full RF cycle were averaged to give the totals shown in 


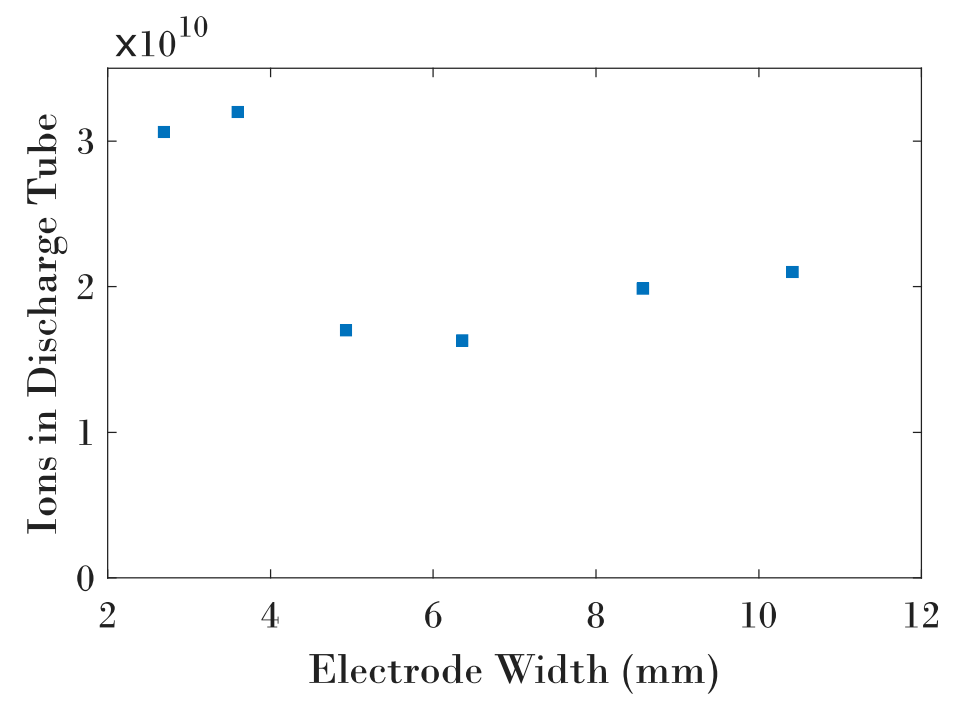

\section{Figure 3.7: Total Ion Quantity vs Electrode Width}

Figure 3.7.

Clearly electrode geometry has a strong effect on the number of ions generated within the discharge. If this CFD model holds true for the 2 and $3 \mathrm{~mm}$ geometries, these appear to be the best choice for generating the most ion-rich discharge, generating $88 \%$ more ions than the baseline design. However, if the results of the model are not accurate for these sizes, the $10 \mathrm{~mm}$ geometry should be selected instead, as it has the next highest quantity of ions. Though it is less of a monumental increase than the possible improvement from the $3 \mathrm{~mm}$ geometry, it still represents a $23 \%$ increase in ion production compared to the baseline $5 \mathrm{~mm}$ case.

Digging into the details of the CFD reveals possible explanations for the trends in Figure 3.7. Firstly, there is a clear trend in the $5-10 \mathrm{~mm}$ electrode sizes of increasing plasma density. This is despite the decrease in voltage applied and the corresponding decrease in $\gamma$. The reason for the observed increase can be clearly seen in Figure 3.8, which compares electron number densities of selected simulations.

Figure 3.8 shows two clear trends: the expected decrease in plasma density with increasing electrode width, and an increase in the width of the discharge with the 

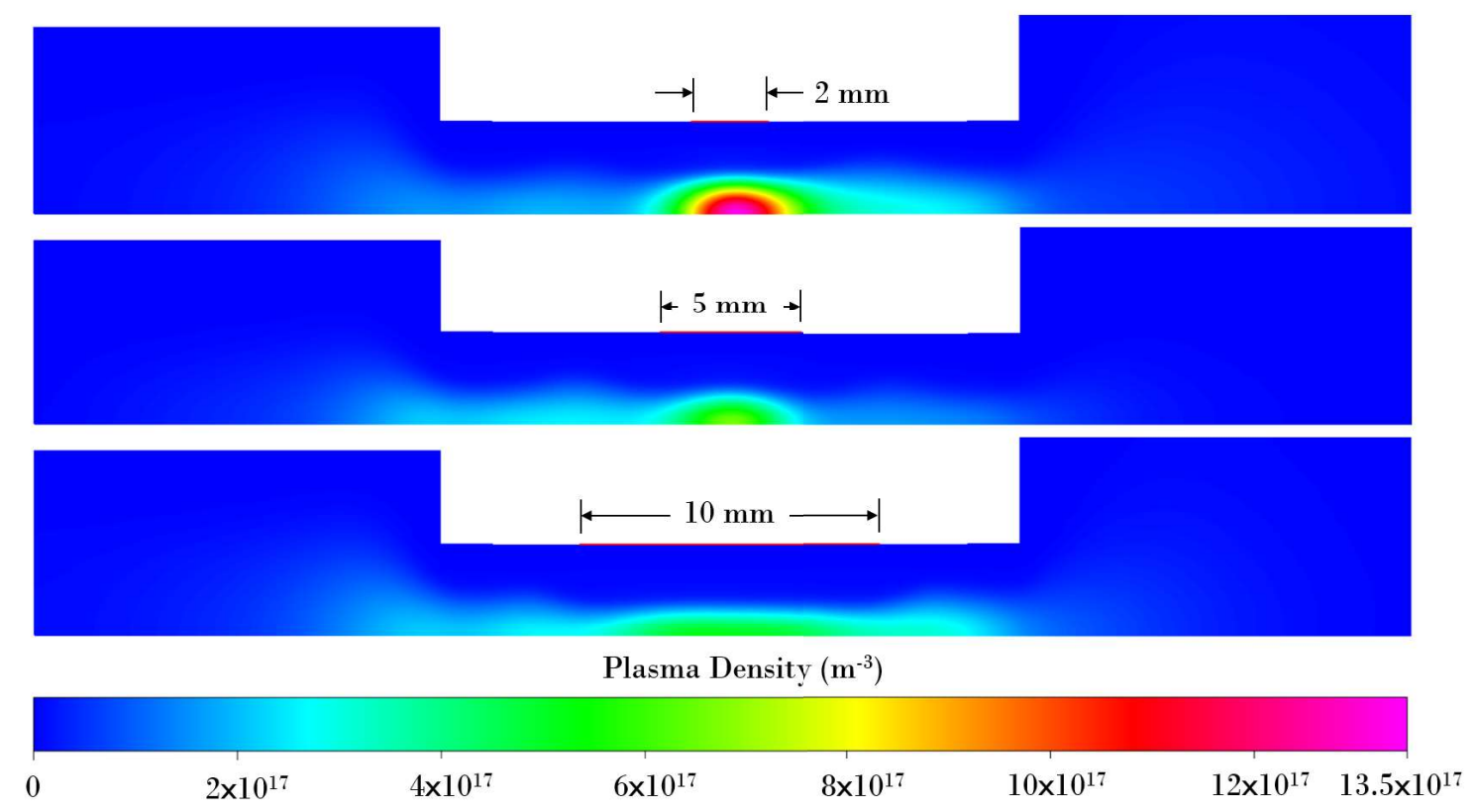

Figure 3.8: Comparison of Electron Densities

width of the electrode. The trend in Figure 3.7 demonstrates that, at least in the 5$10 \mathrm{~mm}$ range, the decreasing intensity of the discharge is overpowered by the increase in discharge size, resulting in a net increase in the number of ions in the discharge.

This comparison of electron densities fails to explain the behavior at the far left of the graph, though. For this, a comparison of peak electron temperature distributions is presented in Figure 3.9. Note that the top simulation is now the $3 \mathrm{~mm}$ case instead of the $2 \mathrm{~mm}$ case to better show the change directly across the discontinuity in Figure 3.7 .

At first glance, there are no significant differences to be seen. The peak electron temperature in the sheath increases as the voltage applied to the thruster increases, which is the expected behavior. The key difference lies in the magnitude of the electron temperatures; the $\mathrm{T}_{e}$ of the $5 \mathrm{~mm}$ simulation maxes out at almost $25 \mathrm{eV}$, whereas the $\mathrm{T}_{e}$ in the $3 \mathrm{~mm}$ case exceeds $30 \mathrm{eV}$. Since the first ionization potential of argon is just over $15 \mathrm{eV}$, the $3 \mathrm{~mm}$ case creates many more electrons capable of 



\section{Figure 3.9: Comparison of Electron Temperatures}

creating two ions each, which significantly boosts the plasma density. However, the experimental data do not seem to display as significant of a spike in plasma density as the CFD results. This was briefly suspected to be because the reaction model used in these simulations did not include the ability to generate doubly ionized species, despite the second ionization potential of Argon also being close to $30 \mathrm{eV}$. This theory was quickly dismissed, though, since only $0.001 \%$ of the argon atoms in the discharge tube are ions. Further, although no data for argon was readily available, the ionization cross section for neon ions is roughly $1 / 10$ th that of neutral neon atoms for electrons with approximately the energies seen in this discharge [48], and the same ratio applies for excitation cross sections of Argon [49]. Therefore, two-step Ar ++ production should be a full six orders of magnitude less prevalent than single ionization. Further, the threshold for double ionization of argon by a single electron impact is nearly $50 \mathrm{eV}$, so this will not affect results either [50]. This finding lends credibility to the observed spike in exhaust plasma density in the $3 \mathrm{~mm}$ experiment 
that previously seemed to be experimental noise, suggesting that there is a measurable benefit to producing electrons with enough energy for two ionizations. Reasons that the CFD model still seems to over-predict this spike could involve the low fidelity modeling of excitation processes and stepwise collisions, or issues with the behavior of the sheath physics model at higher potential gradients.

Also of note is the increase in electron temperature on the downstream side of the $10 \mathrm{~mm}$ electrode. Gas density in the downstream region of the thruster is notably lower than elsewhere in the device, suggesting that operating pressure may be a way to influence the electron temperature in the discharge. This is likely because decreasing pressure decreases the neutral population within the sheath, making it more likely for secondary electrons to be accelerated all the way through the sheath before participating in collisions.

Another parameter investigated was the total number of excited neutrals present in the discharge tube. Excited neutrals are created from the same electron-neutral collisions that generate ions, but occur when the electron participating in the collision has insufficient energy to fully ionize the impacted neutral. Unless the excited neutral undergoes one or more additional collisions before decaying and becomes ionized, the energy stored in the excited neutral is lost via photon emission and does not contribute useful energy to the thruster. For this reason, a large population of excited neutrals could be a sign of significant energy waste, and should be avoided. Using the same process as before, the total number of excited neutrals in the discharge tube was calculated for each geometry, and is plotted in Figure 3.10.

Unlike with ion production, the electrode geometry does not appear to make a significant difference in excited neutral production. A slight peak in excited atom production is apparent at the $5 \mathrm{~mm}$ test, where most electrons have more than enough energy to ionize a single atom, but not enough to ionize two. This makes participation 


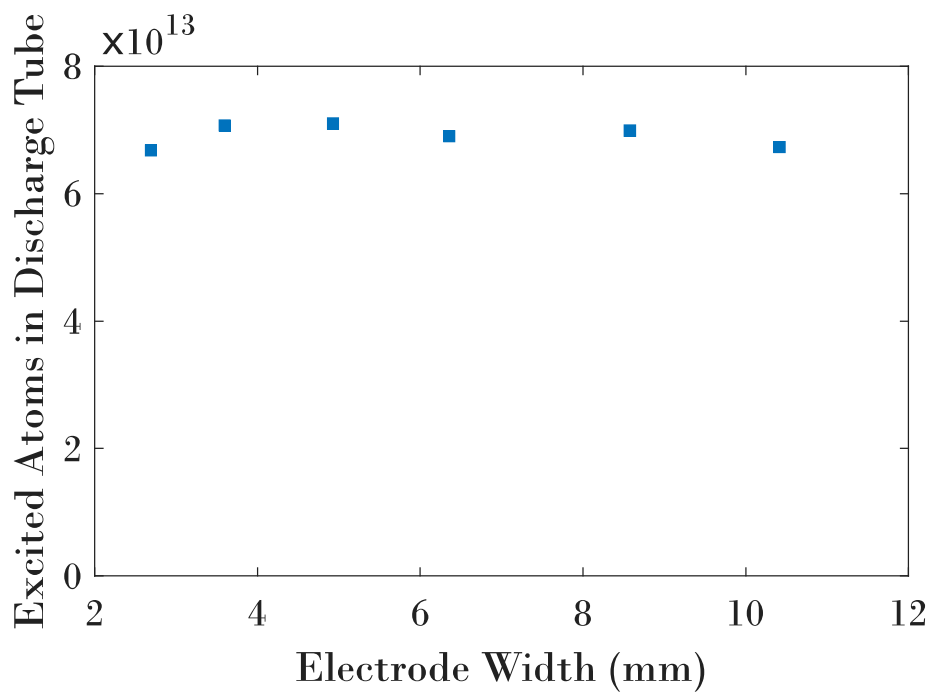

\section{Figure 3.10: Total Excited Neutral Quantity vs Electrode Width}

in an excitation collision more likely for these once-collided electrons. As before with plasma density, excited atom densities are plotted for three selected simulations in Figure 3.11.

As was expected from Figure 3.10, no significant differences in the magnitudes of excited atom density are visible in these plots. The highest peak density out of the three simulations shown is in the $2 \mathrm{~mm}$ case, with a density near $4.2 \times 10^{19}$. The $5 \mathrm{~mm}$ and $10 \mathrm{~mm}$ cases both top out near $4.0 \times 10^{19}$. Because all simulated cases are so similar in excited atom generation, though, it does not appear necessary to consider the number of excited atoms produced when selecting an electrode geometry. Choosing a geometry by plasma density alone simplifies the process, and should still yield optimal results. 

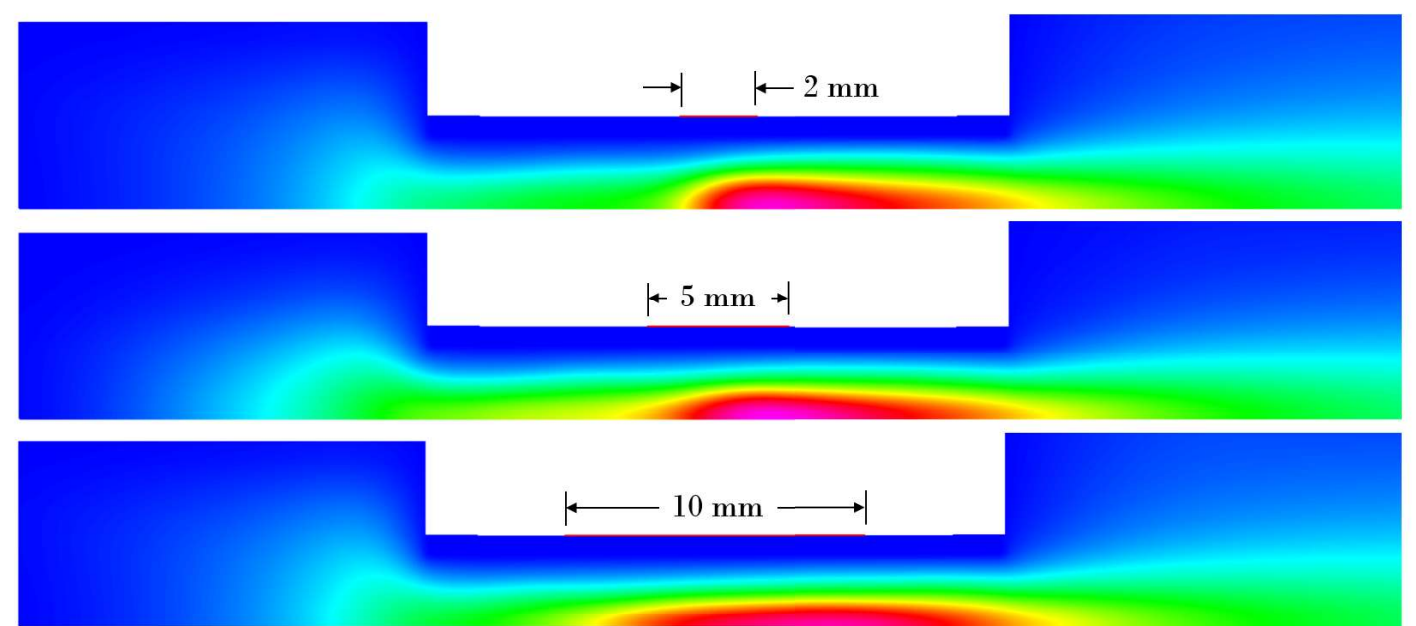

Excited Atom Density $\left(\mathrm{m}^{-3}\right)$

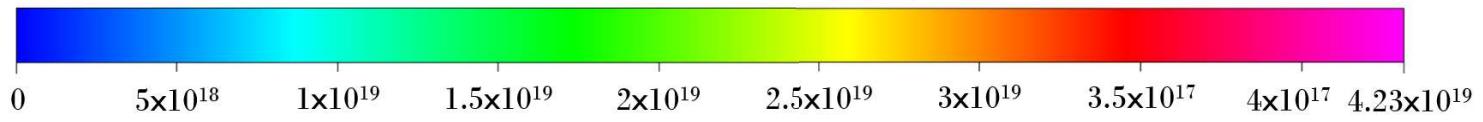

Figure 3.11: Comparison of Excited Atom Density 
Chapter 4

CONCLUSION

The first study investigating the effects of geometry modifications to the Pocket Rocket thruster was conducted, focusing specifically on the impacts of RF electrode size. Six geometries were built and tested in a lab to produce plasma density measurements at the thruster's outlet. These measurements were then used in conjunction with results of prior studies to validate a CFD model of the thruster across the range of tested geometries. From this model, the total quantity of argon ions in the discharge was computed, offering a way to compare the relative effectiveness of each geometry, since ion-neutral charge exchange collisions generate the majority of heating, and therefore thrust, in the device. The configuration with the highest performance in CFD generated $88 \%$ more ions than the baseline geometry by accelerating secondary electrons to sufficient energies to ionize two neutral atoms. The best-performing single ionization-based model still demonstrated a $23 \%$ increase in ion production compared to the baseline, demonstrating that increasing the volume occupied by plasma is a more effective method for increasing plasma quantity with a fixed power input than using a smaller, more concentrated plasma (until the double ionization threshold is reached, that is). This second geometry offers the additional benefit of a $17 \%$ lower operating voltage, which will somewhat decrease the risk of stray discharges in the power conditioning system for the thruster.

It is important to note, though, that the transition from generating electrons that generate only one ion each to electrons that generate two is not a direct consequence of the geometry, but rather the voltage applied to the electrode. It is because these tests took place with a constant power applied that narrower electrodes resulted in higher applied voltages. When designing Pocket Rocket thrusters to specific power 
targets in the future, it is the author's recommendation that the system be designed to operate just above the $200 \mathrm{~V}$ threshold to take advantage of the additional ionization, and that the electrode area be then selected to hit the desired power target. The $200 \mathrm{~V}$ threshold value, it should be noted, only applies to alumina tubes of the same thickness as that used in this study. Unfortunately for anyone wishing to try this design strategy, power consumption as a function of electrode area cannot be reliably extrapolated from these experiments, since the significant power feed losses here would result in an overestimate of thruster power consumption for a more integrated design. This would be a useful area for further research to allow the design of PR thrusters that operate efficiently at the conditions they will be used.

This study also demonstrates the importance of the secondary electron emission coefficient, $\gamma$, in the behavior of these discharges. Future work to further increase the quantity of plasma inside the PR thruster could investigate the performance of various discharge tube materials and/or surface treatments that are likely to increase the quantity of secondary electrons emitted from the wall. $\mathrm{MgO}$, for example, saw widespread use in plasma display panel technology for its high $\gamma$, which is somewhat higher than that of alumina [29].

Further experiments on geometry variations of the Pocket Rocket thruster would also be valuable, including investigations into the use of asymmetric devices (with the RF electrode shifted to one side or another) and investigation into the effects of device diameter. Even without this information, though, the current study represents a significant step towards the creation of a design methodology to create optimal Pocket Rocket thrusters to efficiently and inexpensively propel the next generation of nanosatellites. 


\section{BIBLIOGRAPHY}

[1] Franz Newl, Elliott Coleshill, Ian Dsouza, and Jeff Cain. Nanosatellite Tracking of Ships Review of the First Year of Operations. AIAA 7th Responsive Space Conference, (June 2014), 2009.

[2] Stephen Mauthe, Freddy Pranajaya, and Robert Zee. The design and test of a compact propulsion system for canx nanosatellite formation flying. 2005.

[3] Karan Sarda, Cordell Grant, Stuart Eagleson, Daniel D Kekez, and Robert E Zee. Canadian advanced nanospace experiment 2 orbit operations: two years of pushing the nanosatellite performance envelope. In ESA Small Satellites, Services and Systems Symp, 2010.

[4] Riki Lee, Taylor Lilly, E Muntz, and Andrew Ketsdever. Free molecule micro-resistojet: Nanosatellite propulsion. In 41st AIAA/ASME/SAE/ASEE Joint Propulsion Conference \& Exhibit, page 4073, 2005.

[5] Amelia Diane Greig. Pocket Rocket: An electrothermal plasma micro-thruster. PhD Thesis, (Australian National University), 2015.

[6] Christine Charles, Roderick W. Boswell, Andrew Bish, Vadim Khayms, and Edwin F. Scholz. Direct Measurement of Axial Momentum Imparted by an Electrothermal Radiofrequency Plasma Micro-Thruster. Frontiers in Physics, 4(May):1-6, 2016.

[7] VACCO Industries, 10350 Vacco Street, South El Monte, CA 91733. ArgoMoon Propulsion System, July 2017. 
[8] Busek Co. Inc., 11 Tech Circle, Natick, MA 01760. Busek Micro Resistojet, 2013.

[9] Tethers Unlimited Inc., 11711 N. Creek Pkwy S., D113, Bothell WA 98011. HYDROS-C Green High-Performance Propulsion for Small Satellites, August 2017.

[10] Busek Co. Inc., 11 Tech Circle, Natick, MA 01760. BmP-220 Micro-Pulsed Plasma Thruster, 2015.

[11] Busek Co. Inc., 11 Tech Circle, Natick, MA 01760. BET-1mN Busek Electrospray Thruster, 2016.

[12] Accion Systems Inc. TILE Next Generation Ion Propulsion, 2017.

[13] Deep Space Industries Inc. DSI Comet-1 CubeSat and Microsatellite Water Thrusters, 2016.

[14] Enpulsion. Introduction to the IFM Nano Thruster.

[15] Phase Four Inc. Phase Four Radio Frequency Thruster, May 2017.

[16] Busek Co. Inc., 11 Tech Circle, Natick, MA 01760. BGT-X5 Green Monopropellant Thruster, 2016.

[17] José A Bittencourt. Fundamentals of plasma physics. Springer Science \& Business Media, 2013.

[18] Boris M Smirnov. Physics of ionized gases. John Wiley \& Sons, 2008.

[19] Pascal Chabert and Nicholas Braithwaite. Physics of radio-frequency plasmas. Cambridge University Press, 2011.

[20] Dan M Goebel and Ira Katz. Fundamentals of electric propulsion: ion and Hall thrusters, volume 1. John Wiley \& Sons, 2008. 
[21] AR Ellingboe and RW Boswell. Capacitive, inductive and helicon-wave modes of operation of a helicon plasma source. Physics of Plasmas, 3(7):2797-2804, 1996.

[22] Roderick William Boswell. Very efficient plasma generation by whistler waves near the lower hybrid frequency. Plasma Physics and Controlled Fusion, 26(10):1147, 1984.

[23] JJ Shi and Michael G Kong. Mechanisms of the $\alpha$ and $\gamma$ modes in radio-frequency atmospheric glow discharges. Journal of applied physics, 97(2):023306, 2005.

[24] Raúl A Baragiola. Principles and mechanisms of ion induced electron emission. Nuclear Instruments and Methods in Physics Research Section B: Beam Interactions with Materials and Atoms, 78(1-4):223-238, 1993.

[25] Hannspeter Winter and Joachim Burgdörfer. Slow heavy-particle induced electron emission from solid surfaces, volume 225. Springer, 2007.

[26] V Baglin, J Bojko, C Scheuerlein, Oswald Gröbner, M Taborelli, Bernard Henrist, and Noël Hilleret. The secondary electron yield of technical materials and its variation with surface treatments. Technical report, 2000.

[27] Eun-Ha Choi, Hyun-Joo Oh, Young-Guon Kim, Jae-Jun Ko, Jae-Yong Lim, Jin-Goo Kim, Dae-Il Kim, Guangsup Cho, and Seung-Oun Kang. Measurement of secondary electron emission coefficient $(\gamma)$ of mgo protective layer with various crystallinities. Japanese journal of applied physics, 37(12S):7015, 1998.

[28] Kyoung Sup Moon, Jihwa Lee, and Ki-Woong Whang. Electron ejection from mgo thin films by low energy noble gas ions: Energy dependence and initial 
instability of the secondary electron emission coefficient. Journal of applied physics, 86(7):4049-4051, 1999.

[29] TJ Vink, AR Balkenende, RGFA Verbeek, HAM Van Hal, and ST De Zwart. Materials with a high secondary-electron yield for use in plasma displays. Applied physics letters, 80(12):2216-2218, 2002.

[30] Teck Seng Ho, Christine Charles, Rod Boswell, Teck Seng Ho, Christine Charles, and Rod Boswell. Neutral gas heating and ion transport in a constricted plasma flow. 084501:8-13, 2017.

[31] HB Smith, C Charles, RW Boswell, and H Kuwahara. Bias formation in a pulsed radiofrequency argon discharge. Journal of applied physics, 82(2):561-565, 1997.

[32] C Charles, R W Boswell, and K Takahashi. Investigation of radiofrequency plasma sources for space travel. Plasma Physics and Controlled Fusion, 54(12):124021, 2012.

[33] A. Greig, C. Charles, R. Hawkins, and R. Boswell. Direct measurement of neutral gas heating in a radio-frequency electrothermal plasma micro-thruster. Applied Physics Letters, 103(7):10-14, 2013.

[34] C. Charles and R. W. Boswell. Measurement and modelling of a radiofrequency micro-thruster. Plasma Sources Science and Technology, 21(2):5-9, 2012.

[35] William CAMPBELL. Outgassing data for selecting spacecraft materials. NASA Reference Publication 1124, 1993.

[36] W. John Wolfgong. The silicone conundrum part ii: "low outgassing" silicones. Journal of Failure Analysis and Prevention, 11(3):215-221, Jun 2011. 
[37] Harold M Mott-Smith. The theory of collectors in gaseous discharges. In Electrical Discharge, pages 99-132. Elsevier, 1961.

[38] Isaac D Sudit and Francis F Chen. Rf compensated probes for high-density discharges. Plasma Sources Science and Technology, 3(2):162, 1994.

[39] A. Ganguli, B. B. Sahu, and R. D. Tarey. A new structure for RF-compensated Langmuir probes with external filters tunable in the absence of plasma. Plasma Sources Science and Technology, 17(1), 2008.

[40] Langmuir probe measurements of weakly collisional electropositive RF discharge plasmas Langmuir probe measurements in electronegative plasmas Multiple harmonic compensation of Langmuir probes in rfdischarges Langmuir probe measurements of weakly collision. Journal of Physics D: Applied Physics J. Phys. D: Appl. Phys. J. Phys. D: Appl. Phys, 34(34):1491-1491, 2001.

[41] Francis F Chen. Langmuir probe diagnostics. In IEEE-ICOPS Meeting, Jeju, Korea, volume 2, 2003.

[42] Francis F Chen, John D Evans, and Wade Zawalski. Electric probes. In In Plasma Diagnostic Techniques, edited by Huddlestone, RH and Leornard, SL. Citeseer, 1965.

[43] Francis F Chen, John D Evans, and Donald Arnush. A floating potential method for measuring ion density. Physics of Plasmas, 9(4):1449-1455, 2002.

[44] Francis F Chen. Langmuir probe analysis for high density plasmas. Physics of Plasmas, 8(6):3029-3041, 2001. 
[45] Göran Norlén. Wavelengths and energy levels of ar i and ar ii based on new interferometric measurements in the region 3 400-9 800 å. Physica Scripta, 8(6):249, 1973.

[46] ESI Group. ESI CFD-ACE+ User Manual, UA/CFD_/16/20/00/A edition, February 2017.

[47] Insook Lee, DB Graves, and MA Lieberman. Modeling electromagnetic effects in capacitive discharges. Plasma Sources Science and Technology, 17(1):015018, 2008.

[48] Lee Joseph Kieffer and Gordon H Dunn. Electron impact ionization cross-section data for atoms, atomic ions, and diatomic molecules: I. experimental data. Reviews of Modern Physics, 38(1):1, 1966.

[49] Joseph M Ajello, Geoffrey K James, Brian Franklin, and Simon Howell. Study of electron impact excitation of argon in the extreme ultraviolet: Emission cross section of resonance lines of ar i, ar ii. Journal of Physics B: Atomic, Molecular and Optical Physics, 23(23):4355, 1990.

[50] P McCallion, M B Shah, and H B Gilbody. A crossed beam study of the multiple ionization of argon by electron impact. Journal of Physics B: Atomic, Molecular and Optical Physics, 25(5):1061, 1992. 


\section{APPENDICES}

\section{A Thruster Operation Procedures}

For possible use in an undergraduate lab class.

\section{Setup}

(a) Clear out Big Green (the vacuum chamber).

(b) Connect the hose barb on top of thruster to the plenum pressure gage through one of the Swagelok feedthroughs.

(c) Connect the hose barb on the rear of the thruster to the argon feed line through another Swagelok feedthrough.

(d) Connect the RF power feed to the appropriate BNC feedthrough on the chamber. Make sure to use one of the coax cables with yellow ends, as these have 50 of characteristic impedance. Most of the black BNC cables in the lab are RG59, which is 75 cable. One of the 50 cables is included in the box of thruster hardware that has spent several hours under vacuum already.

(e) Connect the SMA jack on the top of the thruster to the RF feed. Tighten this as much as possible by hand. This should result in the flange of the SMA connector on the thruster hovering slightly above the aluminum body.

(f) Position the Langmuir probe in its holder such that the tip is flush with the aft face of the thruster and the probe is in the center of the thruster.

(g) Connect the Langmuir probe to the appropriate BNC feedthrough.

(h) Position the thruster as desired in the vacuum chamber. 
(i) Give the SMA connector on the thruster a firm press to close the gap between the connector flange and thruster as much as possible. Some gap should still remain. If no gap is visible, the SMA connector must be tightened further.

(j) Close the vacuum chamber. A roughly equal amount of the lower flange of the chamber should be exposed all the way around the bell. If the two halves appear more than a quarter off of concentric, raise the bell so that there is no contact between the two chamber halves, then push the lower half of the chamber into place. Lifting upward while pushing will help.

(k) Wire the Langmuir probe measurement circuit as described in Chapter 2 of this thesis.

(l) Ensure that the power supply is grounded to the rest of the system (except the oscilloscope).

(m) Check with a multimeter that there is significant resistance $(>20 \mathrm{k} \Omega$ ) between the oscilloscope ground and the system ground.

\section{Running Big Green}

(a) Make sure there is sufficient oil in the vacuum pump by shining a flashlight into the oil reservoir.

(b) Turn the box fan behind the pump on high. Position it as close to the vacuum pump as possible, blowing straight across the center of the pump. This is especially important on hot days.

(c) Turn on the vacuum pump. The pump pressure should drop quickly.

(d) Once the pump pressure drops below 1 Torr, flip the switch to the chamber rough valve. The pressure in the pump will spike, and the pressure in the tank should slowly drop. If any hissing is heard, stop and 
find the leak.

(e) Once the pump gets to between 2 and 10 Torr, it should switch from making a loud rumbling/rattling sound to being much quieter. If the chamber gets below 2 Torr and this transition has not occurred, close the chamber rough valve, turn off the pump, and wait 30 seconds for it to cool down. Repeat as necessary.

(f) Once the chamber has dropped below 2 Torr and the pump is operating quietly, open the argon feed valve (the manual valve closest to the chamber). Open the Argon bottle valve. Slowly turn the dial on the regulator inwards until the pressure in the thruster plenum measures 2 Torr. Maintain this flow for 10 seconds, then unscrew the regulator until flow stops, then close the manual valve. This purges air out of the thruster and feed line.

\section{Running the Thruster}

(a) Turn on the RF power supply and DC voltage source.

(b) Set the power supply to voltage mode. Make sure the output is off.

(c) Set the RF power supply to $10 \mathrm{~W}$ output.

(d) Once the vacuum chamber is below 0.3 Torr, open the manual argon valve two turns.

(e) Turn the regulator inwards until the plenum pressure is 1.3 to 1.5 Torr.

(f) Turn on the RF power output.

(g) Adjust the two tuning knobs on the matching network until the power supply displays $0 \mathrm{~W}$ reflected power.

(h) Use the manual valve knob to set the pressure in the thruster plenum as close as possible to 1.5 Torr. 


\section{Taking Data}

(a) Set the time scale of the oscilloscope to $250 \mathrm{~ms}$ per division.

(b) Set the scale of the channel measuring the power supply to $50 \mathrm{~V}$ per division. Make sure this channel is using a $10 \mathrm{x}$ or $100 \mathrm{x}$ probe, and that this has been entered into the oscilloscope software.

(c) Turn on the DC power supply output

(d) Adjust the voltage back and forth between the maximum and minimum limits, watching how high and low the probe current goes on the oscilloscope. Adjust the gain and bias on this channel of the oscilloscope so that the signal takes up as much of the screen as possible.

(e) Set the power supply to $-100 \mathrm{~V}$.

(f) Set the oscilloscope to capture mode.

(g) Smoothly sweep the voltage coming out of the power supply from -100 V to 100

(h) When $0 \mathrm{~V}$ is crossed, have an assistant manually trigger the oscilloscope.

(i) Turn off the DC supply output.

(j) Examine the recorded trace. If unsuccessful, start again at step (e). If successful, proceed.

(k) Record the vacuum chamber pressure and plenum pressure.

(1) Repeat steps (e) through (k), this time starting at $100 \mathrm{~V}$, then sweeping from $100 \mathrm{~V}$ to $-100 \mathrm{~V}$.

(m) Turn off the RF power output.

(n) Turn off the argon flow by first backing out the regulator, then closing the manual valve. 
(o) Repeat steps (3.d) through (4.n) twice more.

5. Shutting Down

(a) Close the chamber rough valve

(b) Turn off the vacuum pump

(c) Flow argon through the thruster at a plenum pressure of 2 Torr for 10 seconds. This cools the probe before reactive atmospheric gases are allowed to enter.

(d) Shut off the flow of argon, closing the manual valve.

(e) Open the chamber vent valve.

(f) If an auxiliary vent path has been added to the chamber, open this once the chamber pressure has reached 100 Torr.

(g) Turn off the DC power supply

(h) Turn off the RF power supply

(i) If no more tests will be conducted on the same day, turn off the box fan at the pump.

(j) Once the chamber is within 20 Torr of atmospheric pressure, the chamber bell may be lifted. Move the hoist slowly with short button presses as it makes contact

(k) with the bell and lifts it into the air. After this point the bell can be lifted continuously.

(l) Once all required activities have been completed in the bell jar, it must be lowered and stowed in the closed position. 


\section{B Simulation Automation Script}

The simulations included in this thesis require periodic adjustment as they run to converge properly. This script is included to save future researchers following up on this work from either having to make these adjustments manually or write a new script themselves.

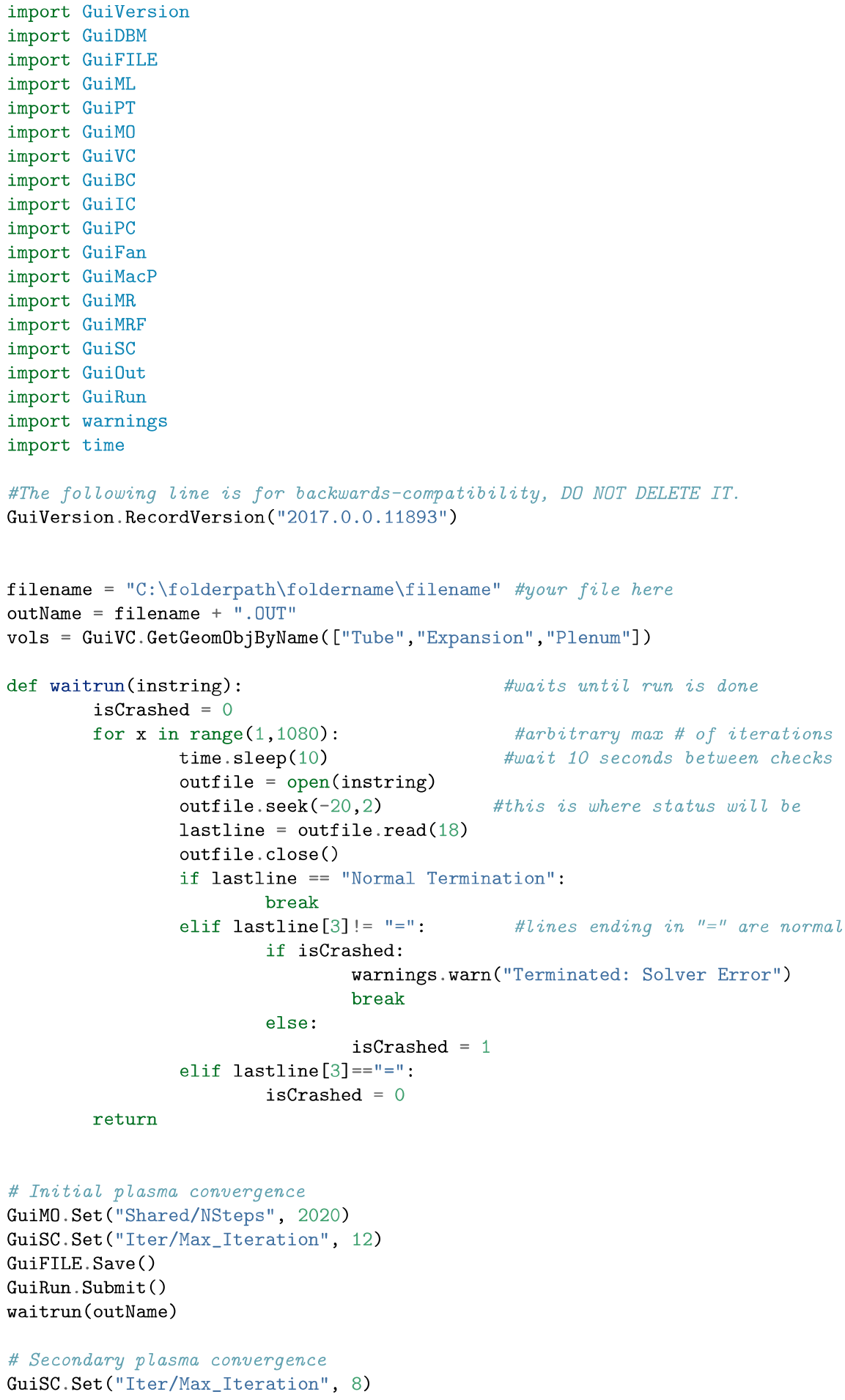


GuiIC.Set ("Shared/IC_Source", "Previous")

GuiIC.Set(vols, "Shared/RestartFile_Name", filename + ".0002020.DTF")

GuiIC.Set ("Shared/ResetTimetoOSecond", 0)

GuiIC.Set ("Shared/AppendResidualFile", 1)

GuiIC.Set ("Shared/AppendOutputFile", 1)

GuiIC.Set ("Shared/AppendMonitorFile", 1)

Guimo.Set ("Shared/NSteps", 3030)

GuiVC.Set(vols, "Plasma/CollisionFrequency_eval_method", "Function of Te")

GuifILE.Save()

GuiRun. Submit()

waitrun (outName)

\# Tertiary plasma convergence

GuiSC.Set("Iter/Max_Iteration", 12)

GuiIC.Set(vols, "Shared/RestartFile_Name", filename + ".0005050.DTF")

GuiMO.Set ("Shared/NSteps", 3030)

GuiFILE.Save()

GuiRun.Submit()

waitrun (outName)

\# Finishing Pass

GuiSC.Set ("Iter/Max_Iteration", 30)

GuiIC.Set(vols, "Shared/RestartFile_Name", filename + ".0008080.DTF")

GuiOut.Set ("Output/Output_Interval", 1)

GuiMO.Set ("Shared/NSteps", 60)

GuiFILE.Save()

GuiRun.Submit () 


\section{Model Setup Script}

This script can be used to exactly duplicate the settings used for the simulations in this thesis.




GuiDBM.Set(iType, iName, "Transport/Dynamic Viscosity/Polynomial Coefficients in Temperature/C4", 0, $\hookrightarrow$ dbLevel)

GuiDBM.Set(iType, iName, "Transport/Dynamic Viscosity/Polynomial Coefficients in Temperature/C5", 0, $\hookrightarrow$ dbLevel)

GuiDBM.Set(iType, iName, "Thermal/Thermal Accomodation Coefficient/Constant/Value", 0.9317, dbLevel) GuiDBM.Set(iType, iName, "Thermal/Specific Heat/JANNAF Coefficients/Temperature Limits/Lower Limit", $\hookrightarrow \quad 300$, dbLevel)

GuiDBM.Set(iType, iName, "Thermal/Specific Heat/JANNAF Coefficients/Temperature Limits/Break Point", $\hookrightarrow \quad 1000$, dbLevel)

GuiDBM.Set(iType, iName, "Thermal/Specific Heat/JANNAF Coefficients/Temperature Limits/Upper Limit", $\hookrightarrow \quad 5000$, dbLevel)

GuiDBM.Set(iType, iName, "Thermal/Specific Heat/JANNAF Coefficients/Coefficients at Lower Limit/a1", $\hookrightarrow \quad 2.5$, dbLevel)

GuiDBM.Set(iType, iName, "Thermal/Specific Heat/JANNAF Coefficients/Coefficients at Lower Limit/a2", $\hookrightarrow \quad 0$, dbLevel)

GuiDBM.Set(iType, iName, "Thermal/Specific Heat/JANNAF Coefficients/Coefficients at Lower Limit/a3", $\hookrightarrow \quad 0$, dbLevel)

GuiDBM.Set(iType, iName, "Thermal/Specific Heat/JANNAF Coefficients/Coefficients at Lower Limit/a4", $\hookrightarrow \quad 0$, dbLevel)

GuiDBM.Set(iType, iName, "Thermal/Specific Heat/JANNAF Coefficients/Coefficients at Lower Limit/a5", $\hookrightarrow \quad 0$, dbLevel)

GuiDBM.Set(iType, iName, "Thermal/Specific Heat/JANNAF Coefficients/Coefficients at Lower Limit/a6", $\hookrightarrow \quad-745.375$, dbLevel)

GuiDBM.Set(iType, iName, "Thermal/Specific Heat/JANNAF Coefficients/Coefficients at Lower Limit/a7", $\hookrightarrow \quad 4.366001$, dbLevel)

GuiDBM.Set(iType, iName, "Thermal/Specific Heat/JANNAF Coefficients/Coefficients at Upper Limit/a1", $\hookrightarrow \quad 2.5$, dbLevel)

GuiDBM.Set(iType, iName, "Thermal/Specific Heat/JANNAF Coefficients/Coefficients at Upper Limit/a2", $\hookrightarrow \quad 0$, dbLevel)

GuiDBM.Set(iType, iName, "Thermal/Specific Heat/JANNAF Coefficients/Coefficients at Upper Limit/a3", $\hookrightarrow \quad 0$, dbLevel)

GuiDBM.Set(iType, iName, "Thermal/Specific Heat/JANNAF Coefficients/Coefficients at Upper Limit/a4", $\hookrightarrow \quad 0$, dbLevel)

GuiDBM.Set(iType, iName, "Thermal/Specific Heat/JANNAF Coefficients/Coefficients at Upper Limit/a5", $\hookrightarrow \quad 0$, dbLevel)

GuiDBM.Set(iType, iName, "Thermal/Specific Heat/JANNAF Coefficients/Coefficients at Upper Limit/a6", $\hookrightarrow \quad-745.375$, dbLevel)

GuiDBM.Set(iType, iName, "Thermal/Specific Heat/JANNAF Coefficients/Coefficients at Upper Limit/a7", $\hookrightarrow \quad 4.366001$, dbLevel)

GuiDBM.Set(iType, iName, "Thermal/Specific Heat/Polynomial Coefficients in Temperature/CO", 0, $\hookrightarrow$ dbLevel)

GuiDBM.Set(iType, iName, "Thermal/Specific Heat/Polynomial Coefficients in Temperature/C1", 0, $\hookrightarrow$ dbLevel)

GuiDBM.Set(iType, iName, "Thermal/Specific Heat/Polynomial Coefficients in Temperature/C2", 0, $\hookrightarrow$ dbLevel)

GuiDBM.Set(iType, iName, "Thermal/Specific Heat/Polynomial Coefficients in Temperature/C3", 0, $\hookrightarrow$ dbLevel)

GuiDBM.Set(iType, iName, "Thermal/Specific Heat/Polynomial Coefficients in Temperature/C4", 0, $\hookrightarrow$ dbLevel)

GuiDBM.Set(iType, iName, "Thermal/Specific Heat/Polynomial Coefficients in Temperature/C5", 0, $\hookrightarrow$ dbLevel)

GuiDBM.Set(iType, iName, "Thermal/Conductivity/Polynomial Coefficients in Temperature/Co", 0, dbLevel) GuiDBM.Set(iType, iName, "Thermal/Conductivity/Polynomial Coefficients in Temperature/C1", 0, dbLevel) GuiDBM.Set(iType, iName, "Thermal/Conductivity/Polynomial Coefficients in Temperature/C2", 0, dbLevel) GuiDBM.Set (iType, iName, "Thermal/Conductivity/Polynomial Coefficients in Temperature/C3", 0, dbLevel) GuiDBM.Set(iType, iName, "Thermal/Conductivity/Polynomial Coefficients in Temperature/C4", 0, dbLevel) GuiDBM.Set(iType, iName, "Thermal/Conductivity/Polynomial Coefficients in Temperature/C5", 0, dbLevel) GuiDBM.Set(iType, iName, "Chemical/Mass Diffusivity/Polynomial Coefficients in Temperature/C0", 0.003, $\hookrightarrow$ dbLevel)

GuiDBM.Set(iType, iName, "Chemical/Mass Diffusivity/Polynomial Coefficients in Temperature/C1", 0, $\hookrightarrow$ dbLevel)

GuiDBM.Set(iType, iName, "Chemical/Mass Diffusivity/Polynomial Coefficients in Temperature/C2", 0, $\hookrightarrow$ dbLevel)

GuiDBM.Set(iType, iName, "Chemical/Mass Diffusivity/Polynomial Coefficients in Temperature/C3", 0, $\hookrightarrow$ dbLevel)

GuiDBM.Set(iType, iName, "Chemical/Mass Diffusivity/Polynomial Coefficients in Temperature/C4", 0, $\hookrightarrow$ dbLevel) 
GuiDBM.Set(iType, iName, "Chemical/Mass Diffusivity/Polynomial Coefficients in Temperature/C5", 0, $\hookrightarrow$ dbLevel)

GuiDBM.Set(iType, iName, "Chemical/Mobility/Polynomial Coefficients in Temperature/Co", 0, dbLevel) GuiDBM.Set(iType, iName, "Chemical/Mobility/Polynomial Coefficients in Temperature/C1", 0, dbLevel) GuiDBM.Set(iType, iName, "Chemical/Mobility/Polynomial Coefficients in Temperature/C2", 0, dbLevel) GuiDBM.Set(iType, iName, "Chemical/Mobility/Polynomial Coefficients in Temperature/C3", 0, dbLevel) GuiDBM.Set(iType, iName, "Chemical/Mobility/Polynomial Coefficients in Temperature/C4", 0, dbLevel) GuiDBM.Set(iType, iName, "Chemical/Mobility/Polynomial Coefficients in Temperature/C5", 0, dbLevel) \# $A R *$

iType $=$ GuiDBM. SPECIES

iName $=$ "AR*"

dbLevel = GuiDBM. MODEL

GuiDBM.AddItem (iType, iName, dbLevel)

GuiDBM.SetNotes (iType, iName, " ", dbLevel)

GuiDBM.AddToComposition (iType, iName, "Ar", 1, dbLevel)

GuiDBM.Set(iType, iName, "Physical-Chemical/Molecular Weight/Constant/M", 39.9481, dbLevel)

GuiDBM.Set(iType, iName, "Physical-Chemical/Characteristic Energy/Constant/Value", 93.3, dbLevel)

GuiDBM.Set(iType, iName, "Physical-Chemical/Collision Diameter/Constant/Value", 3.542, dbLevel)

GuiDBM.Set(iType, iName, "Physical-Chemical/Polarizability/Constant/Value", 1.642, dbLevel)

GuidBM.Set(iType, iName, "Physical-Chemical/Charge Exchange Cross Section/Constant/Value", 49, $\hookrightarrow$ dbLevel)

GuiDBM.Set(iType, iName, "Physical/Density/Polynomial Coefficients in Temperature/C0", 0, dbLevel)

GuiDBM.Set (iType, iName, "Physical/Density/Polynomial Coefficients in Temperature/C1", 0, dbLevel)

GuiDBM.Set(iType, iName, "Physical/Density/Polynomial Coefficients in Temperature/C2", 0, dbLevel)

GuiDBM.Set (iType, iName, "Physical/Density/Polynomial Coefficients in Temperature/C3", 0, dbLevel)

GuiDBM.Set(iType, iName, "Physical/Density/Polynomial Coefficients in Temperature/C4", 0, dbLevel)

GuiDBM.Set(iType, iName, "Physical/Density/Polynomial Coefficients in Temperature/C5", 0, dbLevel)

GuiDBM.Set(iType, iName, "Transport/Accomodation Coefficient/Constant/Value", 0.9317, dbLevel)

GuiDBM.Set(iType, iName, "Transport/Dynamic Viscosity/Sutherland's Law Coefficients/A",

$\hookrightarrow \quad 2.2001956 \mathrm{E}-006$, dbLevel)

GuiDBM.Set(iType, iName, "Transport/Dynamic Viscosity/Sutherland's Law Coefficients/B", 379.32156, $\hookrightarrow$ dbLevel)

GuiDBM.Set(iType, iName, "Transport/Dynamic Viscosity/Polynomial Coefficients in Temperature/Co", 0, $\hookrightarrow$ dbLevel)

GuiDBM.Set(iType, iName, "Transport/Dynamic Viscosity/Polynomial Coefficients in Temperature/C1", 0, $\hookrightarrow$ dbLevel)

GuiDBM.Set(iType, iName, "Transport/Dynamic Viscosity/Polynomial Coefficients in Temperature/C2", 0, $\hookrightarrow$ dbLevel)

GuiDBM.Set(iType, iName, "Transport/Dynamic Viscosity/Polynomial Coefficients in Temperature/C3", 0, $\hookrightarrow$ dbLevel)

GuiDBM.Set(iType, iName, "Transport/Dynamic Viscosity/Polynomial Coefficients in Temperature/C4", 0, $\hookrightarrow$ dbLevel)

GuiDBM.Set(iType, iName, "Transport/Dynamic Viscosity/Polynomial Coefficients in Temperature/C5", 0, $\hookrightarrow$ dbLevel)

GuiDBM.Set(iType, iName, "Thermal/Thermal Accomodation Coefficient/Constant/Value", 0.9317, dbLevel)

GuiDBM.Set(iType, iName, "Thermal/Specific Heat/JANNAF Coefficients/Temperature Limits/Lower Limit", $\hookrightarrow \quad 100$, dbLevel)

GuiDBM.Set(iType, iName, "Thermal/Specific Heat/JANNAF Coefficients/Temperature Limits/Break Point", $\hookrightarrow \quad 1000$, dbLevel)

GuiDBM.Set(iType, iName, "Thermal/Specific Heat/JANNAF Coefficients/Temperature Limits/Upper Limit", $\hookrightarrow \quad 5000$, dbLevel)

GuiDBM.Set(iType, iName, "Thermal/Specific Heat/JANNAF Coefficients/Coefficients at Lower Limit/a1", $\hookrightarrow \quad 2.5002516$, dbLevel)

GuiDBM.Set(iType, iName, "Thermal/Specific Heat/JANNAF Coefficients/Coefficients at Lower Limit/a2", $\hookrightarrow \quad 0$, dbLevel)

GuiDBM.Set(iType, iName, "Thermal/Specific Heat/JANNAF Coefficients/Coefficients at Lower Limit/a3", $\hookrightarrow \quad 0$, dbLevel)

GuiDBM.Set(iType, iName, "Thermal/Specific Heat/JANNAF Coefficients/Coefficients at Lower Limit/a4", $\hookrightarrow \quad 0$, dbLevel)

GuiDBM.Set(iType, iName, "Thermal/Specific Heat/JANNAF Coefficients/Coefficients at Lower Limit/a5", $\hookrightarrow \quad 0$, dbLevel)

GuiDBM.Set(iType, iName, "Thermal/Specific Heat/JANNAF Coefficients/Coefficients at Lower Limit/a6", $\hookrightarrow$ 131842, dbLevel)

GuiDBM.Set(iType, iName, "Thermal/Specific Heat/JANNAF Coefficients/Coefficients at Lower Limit/a7", $\hookrightarrow \quad 4.3668517$, dbLevel)

GuiDBM.Set(iType, iName, "Thermal/Specific Heat/JANNAF Coefficients/Coefficients at Upper Limit/a1", $\hookrightarrow \quad 2.5002516$, dbLevel) 
GuiDBM.Set(iType, iName, "Thermal/Specific Heat/JANNAF Coefficients/Coefficients at Upper Limit/a2", $\hookrightarrow \quad 0$, dbLevel)

GuiDBM.Set(iType, iName, "Thermal/Specific Heat/JANNAF Coefficients/Coefficients at Upper Limit/a3", $\hookrightarrow \quad 0$, dbLevel)

GuiDBM.Set(iType, iName, "Thermal/Specific Heat/JANNAF Coefficients/Coefficients at Upper Limit/a4", $\hookrightarrow \quad 0$, dbLevel)

GuiDBM.Set(iType, iName, "Thermal/Specific Heat/JANNAF Coefficients/Coefficients at Upper Limit/a5", $\hookrightarrow \quad 0$, dbLevel)

GuiDBM.Set(iType, iName, "Thermal/Specific Heat/JANNAF Coefficients/Coefficients at Upper Limit/a6", $\hookrightarrow \quad 131842$, dbLevel)

GuiDBM.Set(iType, iName, "Thermal/Specific Heat/JANNAF Coefficients/Coefficients at Upper Limit/a7", $\hookrightarrow \quad 4.3668517$, dbLevel)

GuiDBM.Set(iType, iName, "Thermal/Specific Heat/Polynomial Coefficients in Temperature/C0", 0, $\hookrightarrow$ dbLevel)

GuiDBM.Set(iType, iName, "Thermal/Specific Heat/Polynomial Coefficients in Temperature/C1", 0, $\hookrightarrow$ dbLevel)

GuiDBM.Set(iType, iName, "Thermal/Specific Heat/Polynomial Coefficients in Temperature/C2", 0 , $\hookrightarrow$ dbLevel)

GuiDBM.Set(iType, iName, "Thermal/Specific Heat/Polynomial Coefficients in Temperature/C3", 0, $\hookrightarrow$ dbLevel)

GuiDBM.Set(iType, iName, "Thermal/Specific Heat/Polynomial Coefficients in Temperature/C4", 0, $\hookrightarrow$ dbLevel)

GuiDBM.Set(iType, iName, "Thermal/Specific Heat/Polynomial Coefficients in Temperature/C5", 0, $\hookrightarrow$ dbLevel)

GuiDBM.Set(iType, iName, "Thermal/Conductivity/Polynomial Coefficients in Temperature/CO", 0, dbLevel) GuiDBM.Set(iType, iName, "Thermal/Conductivity/Polynomial Coefficients in Temperature/C1", 0, dbLevel) GuiDBM.Set(iType, iName, "Thermal/Conductivity/Polynomial Coefficients in Temperature/C2", 0, dbLevel) GuiDBM.Set(iType, iName, "Thermal/Conductivity/Polynomial Coefficients in Temperature/C3", 0, dbLevel) GuiDBM.Set(iType, iName, "Thermal/Conductivity/Polynomial Coefficients in Temperature/C4", 0, dbLevel) GuiDBM.Set(iType, iName, "Thermal/Conductivity/Polynomial Coefficients in Temperature/C5", 0, dbLevel) GuiDBM.Set(iType, iName, "Chemical/Mass Diffusivity/Polynomial Coefficients in Temperature/C0", 0.003, $\hookrightarrow$ dbLevel)

GuiDBM.Set(iType, iName, "Chemical/Mass Diffusivity/Polynomial Coefficients in Temperature/C1", 0 , $\hookrightarrow$ dbLevel)

GuiDBM.Set(iType, iName, "Chemical/Mass Diffusivity/Polynomial Coefficients in Temperature/C2", 0 , $\hookrightarrow$ dbLevel)

GuiDBM.Set(iType, iName, "Chemical/Mass Diffusivity/Polynomial Coefficients in Temperature/C3", 0, $\hookrightarrow$ dbLevel)

GuiDBM.Set(iType, iName, "Chemical/Mass Diffusivity/Polynomial Coefficients in Temperature/C4", 0 , $\hookrightarrow$ dbLevel)

GuiDBM.Set(iType, iName, "Chemical/Mass Diffusivity/Polynomial Coefficients in Temperature/C5", 0, $\hookrightarrow$ dbLevel)

GuiDBM.Set(iType, iName, "Chemical/Mobility/Polynomial Coefficients in Temperature/C0", 0, dbLevel) GuiDBM.Set(iType, iName, "Chemical/Mobility/Polynomial Coefficients in Temperature/C1", 0, dbLevel) GuiDBM.Set(iType, iName, "Chemical/Mobility/Polynomial Coefficients in Temperature/C2", 0, dbLevel) GuiDBM.Set(iType, iName, "Chemical/Mobility/Polynomial Coefficients in Temperature/C3", 0, dbLevel) GuiDBM.Set(iType, iName, "Chemical/Mobility/Polynomial Coefficients in Temperature/C4", 0, dbLevel) GuiDBM.Set(iType, iName, "Chemical/Mobility/Polynomial Coefficients in Temperature/C5", 0, dbLevel) \# AR+

iType $=$ GuiDBM.SPECIES

iName = "AR+"

dbLevel $=$ GuiDBM.MODEL

GuiDBM.AddItem (iType, iName, dbLevel)

GuiDBM.SetNotes (iType, iName, "", dbLevel)

GuiDBM. AddToComposition(iType, iName, "Ar", 1, dbLevel)

GuiDBM.Set(iType, iName, "Physical-Chemical/Molecular Weight/Constant/M", 39.9481, dbLevel)

GuiDBM.Set(iType, iName, "Physical-Chemical/Characteristic Energy/Constant/Value", 93.3, dbLevel)

GuiDBM.Set(iType, iName, "Physical-Chemical/Collision Diameter/Constant/Value", 3.542, dbLevel)

GuiDBM.Set(iType, iName, "Physical-Chemical/Polarizability/Constant/Value", 1.642, dbLevel)

GuiDBM.Set(iType, iName, "Physical-Chemical/Charge Exchange Cross Section/Constant/Value", 49, $\hookrightarrow$ dbLevel)

GuiDBM.Set(iType, iName, "Physical/Density/Polynomial Coefficients in Temperature/C0", 0, dbLevel) GuiDBM.Set(iType, iName, "Physical/Density/Polynomial Coefficients in Temperature/C1", 0, dbLevel) GuiDBM.Set(iType, iName, "Physical/Density/Polynomial Coefficients in Temperature/C2", 0, dbLevel) GuiDBM.Set(iType, iName, "Physical/Density/Polynomial Coefficients in Temperature/C3", 0, dbLevel) GuiDBM.Set(iType, iName, "Physical/Density/Polynomial Coefficients in Temperature/C4", 0, dbLevel) GuiDBM.Set(iType, iName, "Physical/Density/Polynomial Coefficients in Temperature/C5", 0, dbLevel) GuiDBM.Set(iType, iName, "Transport/Accomodation Coefficient/Constant/Value", 0.9317, dbLevel) 
GuiDBM.Set(iType, iName, "Transport/Dynamic Viscosity/Sutherland's Law Coefficients/A", 1.966E-006, $\hookrightarrow$ dbLevel)

GuiDBM.Set(iType, iName, "Transport/Dynamic Viscosity/Sutherland's Law Coefficients/B", 147.47, $\hookrightarrow$ dbLevel)

GuiDBM.Set(iType, iName, "Transport/Dynamic Viscosity/Polynomial Coefficients in Temperature/Co", 0, $\hookrightarrow$ dbLevel)

GuiDBM.Set(iType, iName, "Transport/Dynamic Viscosity/Polynomial Coefficients in Temperature/C1", 0, $\hookrightarrow$ dbLevel)

GuiDBM.Set(iType, iName, "Transport/Dynamic Viscosity/Polynomial Coefficients in Temperature/C2", 0, $\hookrightarrow$ dbLevel)

GuiDBM.Set(iType, iName, "Transport/Dynamic Viscosity/Polynomial Coefficients in Temperature/C3", 0, $\hookrightarrow$ dbLevel)

GuiDBM.Set(iType, iName, "Transport/Dynamic Viscosity/Polynomial Coefficients in Temperature/C4", 0, $\hookrightarrow$ dbLevel)

GuiDBM.Set(iType, iName, "Transport/Dynamic Viscosity/Polynomial Coefficients in Temperature/C5", 0, $\hookrightarrow$ dbLevel)

GuiDBM.Set(iType, iName, "Thermal/Thermal Accomodation Coefficient/Constant/Value", 0.9317, dbLevel)

GuiDBM.Set(iType, iName, "Thermal/Specific Heat/JANNAF Coefficients/Temperature Limits/Lower Limit", $\hookrightarrow \quad 300$, dbLevel)

GuiDBM.Set(iType, iName, "Thermal/Specific Heat/JANNAF Coefficients/Temperature Limits/Break Point", $\hookrightarrow \quad 1000$, dbLevel)

GuiDBM.Set(iType, iName, "Thermal/Specific Heat/JANNAF Coefficients/Temperature Limits/Upper Limit", $\hookrightarrow \quad 5000$, dbLevel)

GuiDBM.Set(iType, iName, "Thermal/Specific Heat/JANNAF Coefficients/Coefficients at Lower Limit/a1", $\hookrightarrow \quad 2.301341$, dbLevel)

GuiDBM.Set(iType, iName, "Thermal/Specific Heat/JANNAF Coefficients/Coefficients at Lower Limit/a2", $\hookrightarrow \quad 0.0008035529$, dbLevel)

GuiDBM.Set(iType, iName, "Thermal/Specific Heat/JANNAF Coefficients/Coefficients at Lower Limit/a3", $\hookrightarrow \quad-1.758806 \mathrm{E}-007$, dbLevel)

GuiDBM.Set(iType, iName, "Thermal/Specific Heat/JANNAF Coefficients/Coefficients at Lower Limit/a4", $\hookrightarrow \quad-1.781093 \mathrm{E}-010$, dbLevel)

GuiDBM.Set(iType, iName, "Thermal/Specific Heat/JANNAF Coefficients/Coefficients at Lower Limit/a5", $\hookrightarrow \quad-8.937268 \mathrm{E}-015$, dbLevel)

GuiDBM.Set(iType, iName, "Thermal/Specific Heat/JANNAF Coefficients/Coefficients at Lower Limit/a6", $\hookrightarrow \quad$ 182928.1, dbLevel)

GuiDBM.Set(iType, iName, "Thermal/Specific Heat/JANNAF Coefficients/Coefficients at Lower Limit/a7", $\hookrightarrow$ 6.659358, dbLevel)

GuiDBM.Set(iType, iName, "Thermal/Specific Heat/JANNAF Coefficients/Coefficients at Upper Limit/a1", $\hookrightarrow \quad 2.864864$, dbLevel)

GuiDBM.Set(iType, iName, "Thermal/Specific Heat/JANNAF Coefficients/Coefficients at Upper Limit/a2", $\hookrightarrow \quad-0.0001203573$, dbLevel)

GuiDBM.Set(iType, iName, "Thermal/Specific Heat/JANNAF Coefficients/Coefficients at Upper Limit/a3", $\hookrightarrow \quad-1.065199 \mathrm{E}-008$, dbLevel)

GuiDBM.Set(iType, iName, "Thermal/Specific Heat/JANNAF Coefficients/Coefficients at Upper Limit/a4", $\hookrightarrow$ 9.074839E-012, dbLevel)

GuiDBM.Set(iType, iName, "Thermal/Specific Heat/JANNAF Coefficients/Coefficients at Upper Limit/a5", $\hookrightarrow \quad-9.623876 \mathrm{E}-016$, dbLevel)

GuiDBM.Set(iType, iName, "Thermal/Specific Heat/JANNAF Coefficients/Coefficients at Upper Limit/a6", $\hookrightarrow \quad 182723$, dbLevel)

GuiDBM.Set(iType, iName, "Thermal/Specific Heat/JANNAF Coefficients/Coefficients at Upper Limit/a7", $\hookrightarrow \quad 3.543585$, dbLevel)

GuiDBM.Set(iType, iName, "Thermal/Specific Heat/Polynomial Coefficients in Temperature/CO", 0, $\hookrightarrow$ dbLevel)

GuiDBM.Set(iType, iName, "Thermal/Specific Heat/Polynomial Coefficients in Temperature/C1", 0, $\hookrightarrow$ dbLevel)

GuiDBM.Set(iType, iName, "Thermal/Specific Heat/Polynomial Coefficients in Temperature/C2", 0, $\hookrightarrow$ dbLevel)

GuiDBM.Set(iType, iName, "Thermal/Specific Heat/Polynomial Coefficients in Temperature/C3", 0, $\hookrightarrow$ dbLevel)

GuiDBM.Set(iType, iName, "Thermal/Specific Heat/Polynomial Coefficients in Temperature/C4", 0, $\hookrightarrow$ dbLevel)

GuiDBM.Set(iType, iName, "Thermal/Specific Heat/Polynomial Coefficients in Temperature/C5", 0, $\hookrightarrow$ dbLevel)

GuiDBM.Set(iType, iName, "Thermal/Conductivity/Polynomial Coefficients in Temperature/CO", 0, dbLevel)

GuiDBM.Set(iType, iName, "Thermal/Conductivity/Polynomial Coefficients in Temperature/C1", 0, dbLevel)

GuiDBM.Set(iType, iName, "Thermal/Conductivity/Polynomial Coefficients in Temperature/C2", 0, dbLevel)

GuiDBM.Set (iType, iName, "Thermal/Conductivity/Polynomial Coefficients in Temperature/C3", 0, dbLevel)

GuiDBM.Set(iType, iName, "Thermal/Conductivity/Polynomial Coefficients in Temperature/C4", 0, dbLevel) 
GuiDBM.Set(iType, iName, "Thermal/Conductivity/Polynomial Coefficients in Temperature/C5", 0, dbLevel) GuiDBM.Set(iType, iName, "Chemical/Mass Diffusivity/Polynomial Coefficients in Temperature/C0", 0.003, $\hookrightarrow$ dbLevel)

GuiDBM.Set(iType, iName, "Chemical/Mass Diffusivity/Polynomial Coefficients in Temperature/C1", 0, $\hookrightarrow$ dbLevel)

GuiDBM.Set(iType, iName, "Chemical/Mass Diffusivity/Polynomial Coefficients in Temperature/C2", 0, $\hookrightarrow$ dbLevel)

GuiDBM.Set(iType, iName, "Chemical/Mass Diffusivity/Polynomial Coefficients in Temperature/C3", 0, $\hookrightarrow$ dbLevel)

GuiDBM.Set(iType, iName, "Chemical/Mass Diffusivity/Polynomial Coefficients in Temperature/C4", 0, $\hookrightarrow$ dbLevel)

GuiDBM.Set(iType, iName, "Chemical/Mass Diffusivity/Polynomial Coefficients in Temperature/C5", 0, $\hookrightarrow$ dbLevel)

GuiDBM.Set(iType, iName, "Chemical/Mobility/Polynomial Coefficients in Temperature/Co", 0, dbLevel) GuiDBM.Set(iType, iName, "Chemical/Mobility/Polynomial Coefficients in Temperature/C1", 0, dbLevel) GuiDBM.Set(iType, iName, "Chemical/Mobility/Polynomial Coefficients in Temperature/C2", 0, dbLevel) GuiDBM.Set(iType, iName, "Chemical/Mobility/Polynomial Coefficients in Temperature/C3", 0, dbLevel) GuiDBM.Set(iType, iName, "Chemical/Mobility/Polynomial Coefficients in Temperature/C4", 0, dbLevel) GuiDBM.Set(iType, iName, "Chemical/Mobility/Polynomial Coefficients in Temperature/C5", 0, dbLevel) \# E

iType $=$ GuiDBM.SPECIES

iName = "E"

dbLevel = GuiDBM. MODEL

GuiDBM.AddItem(iType, iName, dbLevel)

GuiDBM.SetNotes (iType, iName, "", dbLevel)

GuiDBM.AddToComposition(iType, iName, "E", 1, dbLevel)

GuiDBM.Set(iType, iName, "Physical-Chemical/Molecular Weight/Constant/M", 0.00054857990981, dbLevel) GuiDBM.Set(iType, iName, "Physical-Chemical/Characteristic Energy/Constant/Value", 37, dbLevel)

GuiDBM.Set(iType, iName, "Physical-Chemical/Collision Diameter/Constant/Value", 2.71, dbLevel)

GuiDBM.Set(iType, iName, "Physical-Chemical/Polarizability/Constant/Value", 1.642, dbLevel)

GuiDBM.Set(iType, iName, "Physical-Chemical/Charge Exchange Cross Section/Constant/Value", 40, $\hookrightarrow$ dbLevel)

GuiDBM.Set(iType, iName, "Physical/Density/Polynomial Coefficients in Temperature/Co", 0, dbLevel) GuiDBM.Set(iType, iName, "Physical/Density/Polynomial Coefficients in Temperature/C1", 0, dbLevel) GuiDBM.Set(iType, iName, "Physical/Density/Polynomial Coefficients in Temperature/C2", 0, dbLevel) GuiDBM.Set (iType, iName, "Physical/Density/Polynomial Coefficients in Temperature/C3", 0, dbLevel) GuiDBM.Set (iType, iName, "Physical/Density/Polynomial Coefficients in Temperature/C4", 0, dbLevel) GuiDBM.Set(iType, iName, "Physical/Density/Polynomial Coefficients in Temperature/C5", 0, dbLevel) GuiDBM.Set(iType, iName, "Transport/Accomodation Coefficient/Constant/Value", 0.9317, dbLevel) GuiDBM.Set(iType, iName, "Transport/Dynamic Viscosity/Sutherland's Law Coefficients/A", 0, dbLevel) GuiDBM.Set(iType, iName, "Transport/Dynamic Viscosity/Sutherland's Law Coefficients/B", 0, dbLevel) GuiDBM.Set(iType, iName, "Transport/Dynamic Viscosity/Polynomial Coefficients in Temperature/C0", 0, $\hookrightarrow \quad$ dbLevel)

GuiDBM.Set(iType, iName, "Transport/Dynamic Viscosity/Polynomial Coefficients in Temperature/C1", 0, $\hookrightarrow$ dbLevel)

GuiDBM.Set(iType, iName, "Transport/Dynamic Viscosity/Polynomial Coefficients in Temperature/C2", 0, $\hookrightarrow$ dbLevel)

GuiDBM.Set(iType, iName, "Transport/Dynamic Viscosity/Polynomial Coefficients in Temperature/C3", 0, $\hookrightarrow$ dbLevel)

GuiDBM.Set(iType, iName, "Transport/Dynamic Viscosity/Polynomial Coefficients in Temperature/C4", 0, $\hookrightarrow$ dbLevel)

GuiDBM.Set(iType, iName, "Transport/Dynamic Viscosity/Polynomial Coefficients in Temperature/C5", 0, $\hookrightarrow$ dbLevel)

GuiDBM.Set (iType, iName, "Thermal/Thermal Accomodation Coefficient/Constant/Value", 0.9317, dbLevel) GuiDBM.Set(iType, iName, "Thermal/Specific Heat/JANNAF Coefficients/Temperature Limits/Lower Limit", $\hookrightarrow \quad 300$, dbLevel)

GuiDBM.Set(iType, iName, "Thermal/Specific Heat/JANNAF Coefficients/Temperature Limits/Break Point", $\hookrightarrow \quad 1000$, dbLevel)

GuiDBM.Set(iType, iName, "Thermal/Specific Heat/JANNAF Coefficients/Temperature Limits/Upper Limit", $\hookrightarrow$ 5000, dbLevel)

GuiDBM.Set(iType, iName, "Thermal/Specific Heat/JANNAF Coefficients/Coefficients at Lower Limit/a1", $\hookrightarrow \quad 2.5$, dbLevel)

GuiDBM.Set(iType, iName, "Thermal/Specific Heat/JANNAF Coefficients/Coefficients at Lower Limit/a2", $\hookrightarrow \quad 0$, dbLevel)

GuiDBM.Set(iType, iName, "Thermal/Specific Heat/JANNAF Coefficients/Coefficients at Lower Limit/a3", $\hookrightarrow \quad 0$, dbLevel)

GuiDBM.Set(iType, iName, "Thermal/Specific Heat/JANNAF Coefficients/Coefficients at Lower Limit/a4", $\hookrightarrow \quad 0$, dbLevel) 
GuiDBM.Set(iType, iName, "Thermal/Specific Heat/JANNAF Coefficients/Coefficients at Lower Limit/a5", $\hookrightarrow \quad 0$, dbLevel)

GuiDBM.Set(iType, iName, "Thermal/Specific Heat/JANNAF Coefficients/Coefficients at Lower Limit/a6", $\hookrightarrow \quad-745.375$, dbLevel)

GuiDBM.Set(iType, iName, "Thermal/Specific Heat/JANNAF Coefficients/Coefficients at Lower Limit/a7", $\hookrightarrow \quad-11.734026$, dbLevel)

GuiDBM.Set(iType, iName, "Thermal/Specific Heat/JANNAF Coefficients/Coefficients at Upper Limit/a1", $\hookrightarrow \quad 2.5$, dbLevel)

GuiDBM.Set(iType, iName, "Thermal/Specific Heat/JANNAF Coefficients/Coefficients at Upper Limit/a2", $\hookrightarrow \quad 0$, dbLevel)

GuiDBM.Set(iType, iName, "Thermal/Specific Heat/JANNAF Coefficients/Coefficients at Upper Limit/a3", $\hookrightarrow \quad 0$, dbLevel)

GuiDBM.Set(iType, iName, "Thermal/Specific Heat/JANNAF Coefficients/Coefficients at Upper Limit/a4", $\hookrightarrow \quad 0$, dbLevel)

GuiDBM.Set(iType, iName, "Thermal/Specific Heat/JANNAF Coefficients/Coefficients at Upper Limit/a5", $\hookrightarrow \quad 0$, dbLevel)

GuiDBM.Set(iType, iName, "Thermal/Specific Heat/JANNAF Coefficients/Coefficients at Upper Limit/a6", $\hookrightarrow \quad-745.37496$, dbLevel)

GuiDBM.Set(iType, iName, "Thermal/Specific Heat/JANNAF Coefficients/Coefficients at Upper Limit/a7", $\hookrightarrow \quad-11.734026$, dbLevel)

GuiDBM.Set(iType, iName, "Thermal/Specific Heat/Polynomial Coefficients in Temperature/CO", 0, $\hookrightarrow$ dbLevel)

GuiDBM.Set(iType, iName, "Thermal/Specific Heat/Polynomial Coefficients in Temperature/C1", 0, $\hookrightarrow$ dbLevel)

GuiDBM.Set(iType, iName, "Thermal/Specific Heat/Polynomial Coefficients in Temperature/C2", 0, $\hookrightarrow$ dbLevel)

GuiDBM.Set(iType, iName, "Thermal/Specific Heat/Polynomial Coefficients in Temperature/C3", 0, $\hookrightarrow$ dbLevel)

GuiDBM.Set(iType, iName, "Thermal/Specific Heat/Polynomial Coefficients in Temperature/C4", 0, $\hookrightarrow$ dbLevel)

GuiDBM.Set(iType, iName, "Thermal/Specific Heat/Polynomial Coefficients in Temperature/C5", 0, $\hookrightarrow$ dbLevel)

GuidBM.Set(iType, iName, "Thermal/Conductivity/Polynomial Coefficients in Temperature/C0", 0, dbLevel) GuiDBM.Set(iType, iName, "Thermal/Conductivity/Polynomial Coefficients in Temperature/C1", 0, dbLevel) GuiDBM.Set(iType, iName, "Thermal/Conductivity/Polynomial Coefficients in Temperature/C2", 0, dbLevel) GuiDBM.Set(iType, iName, "Thermal/Conductivity/Polynomial Coefficients in Temperature/C3", 0, dbLevel) GuiDBM.Set(iType, iName, "Thermal/Conductivity/Polynomial Coefficients in Temperature/C4", 0, dbLevel) GuiDBM.Set(iType, iName, "Thermal/Conductivity/Polynomial Coefficients in Temperature/C5", 0, dbLevel) GuiDBM.Set(iType, iName, "Chemical/Mass Diffusivity/Polynomial Coefficients in Temperature/C0", 0, $\hookrightarrow$ dbLevel)

GuiDBM.Set(iType, iName, "Chemical/Mass Diffusivity/Polynomial Coefficients in Temperature/C1", 0, $\hookrightarrow$ dbLevel)

GuiDBM.Set(iType, iName, "Chemical/Mass Diffusivity/Polynomial Coefficients in Temperature/C2", 0, $\hookrightarrow$ dbLevel)

GuiDBM.Set(iType, iName, "Chemical/Mass Diffusivity/Polynomial Coefficients in Temperature/C3", 0, $\hookrightarrow$ dbLevel)

GuiDBM.Set(iType, iName, "Chemical/Mass Diffusivity/Polynomial Coefficients in Temperature/C4", 0, $\hookrightarrow$ dbLevel)

GuiDBM.Set(iType, iName, "Chemical/Mass Diffusivity/Polynomial Coefficients in Temperature/C5", 0, $\hookrightarrow$ dbLevel)

GuiDBM.Set(iType, iName, "Chemical/Mobility/Polynomial Coefficients in Temperature/Co", 0, dbLevel) GuiDBM.Set(iType, iName, "Chemical/Mobility/Polynomial Coefficients in Temperature/C1", 0, dbLevel) GuiDBM.Set (iType, iName, "Chemical/Mobility/Polynomial Coefficients in Temperature/C2", 0, dbLevel) GuiDBM.Set(iType, iName, "Chemical/Mobility/Polynomial Coefficients in Temperature/C3", 0, dbLevel) GuiDBM.Set(iType, iName, "Chemical/Mobility/Polynomial Coefficients in Temperature/C4", 0, dbLevel) GuiDBM.Set(iType, iName, "Chemical/Mobility/Polynomial Coefficients in Temperature/C5", 0, dbLevel)

\# Argon

iType $=$ GuiDBM.MIXTURE

iName = "Argon"

dbLevel = GuiDBM. MODEL

GuiDBM.AddItem (iType, iName, dbLevel)

GuiDBM.SetNotes (iType, iName, " , dbLevel)

GuiDBM.AddToComposition(iType, iName, "AR", 1, dbLevel)

GuiDBM.Set(iType, iName, "Mixture/UserInput", "Mass Fraction", dbLevel)

\# ArgonIons

iType $=$ GuiDBM.MIXTURE

iName = "ArgonIons"

dbLevel = GuiDBM. MODEL 
GuiDBM.AddItem(iType, iName, dbLevel)

GuiDBM.SetNotes(iType, iName, "", dbLevel)

GuiDBM.AddToComposition(iType, iName, "AR+", 1e-006, dbLevel)

GuiDBM.AddToComposition(iType, iName, "AR", 0.999999, dbLevel)

GuiDBM.Set(iType, iName, "Mixture/UserInput", "Mass Fraction", dbLevel)

\# PLASMA/Argon

iType $=$ GuiDBM.BULKREACTION

iName = "PLASMA/Argon"

dbLevel $=$ GuiDBM. MODEL

GuiDBM.AddItem(iType, iName, dbLevel)

GuiDBM.SetNotes(iType, iName, "Cross sections are taken from JILA database, University of Colorado", $\hookrightarrow$ dbLevel)

GuiDBM.Set(iType, iName, "Type", "MULTI_STEP", dbLevel)

GuiDBM.Set(iType, iName, "Units", "SI", dbLevel)

GuiDBM.Set(iType, iName, "RateType", "GENERAL_RATE", dbLevel)

GuiDBM.AddReactionStep (iType, iName, "AR+E->AR++2E", dbLevel)

GuiDBM.SetListWithIndex(iType, iName, "ReactStep/Name", 1, "", dbLevel)

GuiDBM.SetListWithIndex(iType, iName, "ReactStep/Notes", 1, "ref. JILA", dbLevel)

GuiDBM.SetListWithIndex(iType, iName, "ReactStep/Forward_ConcentrationExponents", [1, 1], 1.000000, $\hookrightarrow$ dbLevel)

GuiDBM.SetListWithIndex(iType, iName, "ReactStep/Forward_ConcentrationExponents", [1, 2], 1.000000, $\hookrightarrow$ dbLevel)

GuiDBM.SetListWithIndex(iType, iName, "ReactStep/Backward_ConcentrationExponents", [1, 1], 0.000000, $\hookrightarrow$ dbLevel)

GuiDBM.SetListWithIndex(iType, iName, "ReactStep/Backward_ConcentrationExponents", [1, 2], 0.000000, $\hookrightarrow$ dbLevel)

GuiDBM.SetListWithIndex(iType, iName, "ReactStep/EnergyLoss", 1, 0, dbLevel)

GuiDBM.SetListWithIndex(iType, iName, "ReactStep/PressureDependent", 1, 0, dbLevel)

GuiDBM.SetListWithIndex(iType, iName, "ReactStep/Type", 1, "Collision_Cross_Section", dbLevel)

GuiDBM.SetListWithIndex(iType, iName, "ReactStep/Forward_CollisionCrossSectionEnergy", 1,

$\hookrightarrow \quad[0,15.8,16,17,18,20,22,23.75,25,26.5,30,32.5,35,37.5,40,50,55,100,150,200,300,500,700,1000,1500,2000,3000,5000,70$ $\hookrightarrow$ dbLevel)

GuiDBM.SetListWithIndex(iType, iName, "ReactStep/Forward_CollisionCrossSection", 1,

$\hookrightarrow \quad[0,0,0.0202,0.134,0.294,0.63,0.93,1.15,1.3,1.45,1.8,1.99,2.17,2.31,2.39,2.53,2.6,2.85,2.52,2.39,2,1.45,1.15,0.86$, $\hookrightarrow$ dbLevel)

GuiDBM.SetListWithIndex(iType, iName, "ReactStep/BackwardRateByDetailedBalance", 1, "CONST_SPECIFIED", $\hookrightarrow$ dbLevel)

GuiDBM.SetListWithIndex(iType, iName, "ReactStep/Backward_CollisionCrossSectionEnergy", 1, [0], $\hookrightarrow$ dbLevel)

GuiDBM.SetListWithIndex(iType, iName, "ReactStep/Backward_CollisionCrossSection", 1, [0], dbLevel) GuiDBM.AddReactionStep (iType, iName, "AR+E->AR*+E", dbLevel)

GuiDBM.SetListWithIndex(iType, iName, "ReactStep/Name", 2, "", dbLevel)

GuiDBM.SetListWithIndex(iType, iName, "ReactStep/Notes", 2, "", dbLevel)

GuiDBM.SetListWithIndex(iType, iName, "ReactStep/Forward_ConcentrationExponents", [2, 1], 1.000000, $\hookrightarrow$ dbLevel)

GuiDBM.SetListWithIndex(iType, iName, "ReactStep/Forward_ConcentrationExponents", [2, 2], 1.000000, $\hookrightarrow$ dbLevel)

GuiDBM.SetListWithIndex(iType, iName, "ReactStep/Backward_ConcentrationExponents", [2, 1], 1.000000, $\hookrightarrow$ dbLevel)

GuiDBM.SetListWithIndex(iType, iName, "ReactStep/Backward_ConcentrationExponents", [2, 2], 1.000000, $\hookrightarrow$ dbLevel)

GuiDBM.SetListWithIndex(iType, iName, "ReactStep/EnergyLoss", 2, 0, dbLevel)

GuiDBM.SetListWithIndex(iType, iName, "ReactStep/PressureDependent", 2, 0, dbLevel)

GuiDBM.SetListWithIndex(iType, iName, "ReactStep/Type", 2, "Arrhenieus", dbLevel)

GuiDBM.SetListWithIndex(iType, iName, "ReactStep/Forward_Ap", 2, 1.2E-014, dbLevel)

GuiDBM.SetListWithIndex (iType, iName, "ReactStep/Forward_n", 2, 0, dbLevel)

GuiDBM.SetListWithIndex (iType, iName, "ReactStep/Forward_p", 2, 0, dbLevel)

GuiDBM.SetListWithIndex(iType, iName, "ReactStep/Forward_Ea/R", 2, 11.94, dbLevel)

GuiDBM.SetListWithIndex(iType, iName, "ReactStep/Forward_UserSub", 2, 0, dbLevel)

GuiDBM.SetListWithIndex(iType, iName, "ReactStep/BackwardRateByEquilibrium", 2, "CONST_SPECIFIED", $\hookrightarrow$ dbLevel)

GuiDBM.SetListWithIndex(iType, iName, "ReactStep/Backward_Ap", 2, 0, dbLevel)

GuiDBM.SetListWithIndex(iType, iName, "ReactStep/Backward_n", 2, 0, dbLevel)

GuiDBM.SetListWithIndex(iType, iName, "ReactStep/Backward_p", 2, 0, dbLevel)

GuiDBM.SetListWithIndex (iType, iName, "ReactStep/Backward_Ea/R", 2, 0, dbLevel)

GuiDBM.SetListWithIndex (iType, iName, "ReactStep/Backward_UserSub", 2, 0, dbLevel)

GuiDBM.AddReactionStep (iType, iName, "AR*+E->AR++2E", dbLevel)

GuiDBM.SetListWithIndex(iType, iName, "ReactStep/Name", 3, "", dbLevel) 
GuiDBM.SetListWithIndex(iType, iName, "ReactStep/Notes", 3, " ", dbLevel)

GuiDBM.SetListWithIndex(iType, iName, "ReactStep/Forward_ConcentrationExponents", [3, 1], 1.000000, $\hookrightarrow$ dbLevel)

GuiDBM.SetListWithIndex(iType, iName, "ReactStep/Forward_ConcentrationExponents", [3, 2], 1.000000, $\hookrightarrow$ dbLevel)

GuiDBM.SetListWithIndex(iType, iName, "ReactStep/Backward_ConcentrationExponents", [3, 1], 1.000000, $\hookrightarrow$ dbLevel)

GuiDBM.SetListWithIndex(iType, iName, "ReactStep/Backward_ConcentrationExponents", [3, 2], 1.000000, $\hookrightarrow$ dbLevel)

GuiDBM.SetListWithIndex(iType, iName, "ReactStep/EnergyLoss", 3, 0, dbLevel)

GuiDBM.SetListWithIndex (iType, iName, "ReactStep/PressureDependent", 3, 0, dbLevel)

GuiDBM.SetListWithIndex (iType, iName, "ReactStep/Type", 3, "Arrhenieus", dbLevel)

GuiDBM.SetListWithIndex (iType, iName, "ReactStep/Forward_Ap", 3, 3E-013, dbLevel)

GuiDBM.SetListWithIndex (iType, iName, "ReactStep/Forward_n", 3, 0.1, dbLevel)

GuiDBM.SetListWithIndex(iType, iName, "ReactStep/Forward_p", 3, 0, dbLevel)

GuiDBM.SetListWithIndex (iType, iName, "ReactStep/Forward_Ea/R", 3, 5.22, dbLevel)

GuiDBM.SetListWithIndex(iType, iName, "ReactStep/Forward_UserSub", 3, 0, dbLevel)

GuiDBM.SetListWithIndex(iType, iName, "ReactStep/BackwardRateByEquilibrium", 3, "CONST_SPECIFIED", $\hookrightarrow$ dbLevel)

GuiDBM.SetListWithIndex (iType, iName, "ReactStep/Backward_Ap", 3, 0, dbLevel)

GuiDBM.SetListWithIndex (iType, iName, "ReactStep/Backward_n", 3, 0, dbLevel)

GuiDBM.SetListWithIndex (iType, iName, "ReactStep/Backward_p", 3, 0, dbLevel)

GuiDBM.SetListWithIndex (iType, iName, "ReactStep/Backward_Ea/R", 3, 0, dbLevel)

GuiDBM.SetListWithIndex (iType, iName, "ReactStep/Backward_UserSub", 3, 0, dbLevel)

GuiDBM.AddReactionStep (iType, iName, "AR+E->AR+E", dbLevel)

GuiDBM.SetListWithIndex (iType, iName, "ReactStep/Name", 4, " ", dbLevel)

GuiDBM.SetListWithIndex(iType, iName, "ReactStep/Notes", 4, "JILA", dbLevel)

GuiDBM.SetListWithIndex(iType, iName, "ReactStep/Forward_ConcentrationExponents", [4, 1], 1.000000, $\hookrightarrow$ dbLevel)

GuiDBM.SetListWithIndex(iType, iName, "ReactStep/Forward_ConcentrationExponents", [4, 2], 1.000000, $\hookrightarrow$ dbLevel)

GuiDBM.SetListWithIndex(iType, iName, "ReactStep/Backward_ConcentrationExponents", [4, 1], 1.000000, $\hookrightarrow$ dbLevel)

GuiDBM.SetListWithIndex(iType, iName, "ReactStep/Backward_ConcentrationExponents", [4, 2], 1.000000, $\hookrightarrow$ dbLevel)

GuiDBM.SetListWithIndex (iType, iName, "ReactStep/EnergyLoss", 4, 0, dbLevel)

GuiDBM.SetListWithIndex(iType, iName, "ReactStep/PressureDependent", 4, 0, dbLevel)

GuiDBM.SetListWithIndex(iType, iName, "ReactStep/Type", 4, "Collision_Cross_Section", dbLevel)

GuiDBM.SetListWithIndex(iType, iName, "ReactStep/Forward_CollisionCrossSectionEnergy", 4,

$\hookrightarrow \quad[0,0.001,0.002,0.003,0.005,0.007,0.0085,0.01,0.015,0.02,0.03,0.04,0.05,0.07,0.1,0.12,0.15,0.17,0.2,0.25,0.3,0.35$, $\hookrightarrow$ dbLevel)

GuiDBM.SetListWithIndex(iType, iName, "ReactStep/Forward_CollisionCrossSection", 4,

$\hookrightarrow \quad[7.5,7.5,7.1,6.7,6.1,5.4,5.05,4.6,3.75,3.25,2.5,2.05,1.73,1.13,0.59,0.4,0.23,0.16,0.103,0.091,0.153,0.235,0.33,0$. $\hookrightarrow$ dbLevel)

GuiDBM.SetListWithIndex(iType, iName, "ReactStep/BackwardRateByDetailedBalance", 4, "CONST_SPECIFIED", $\hookrightarrow$ dbLevel)

GuiDBM.SetListWithIndex(iType, iName, "ReactStep/Backward_CollisionCrossSectionEnergy", 4, [0], $\hookrightarrow$ dbLevel)

GuiDBM.SetListWithIndex(iType, iName, "ReactStep/Backward_CollisionCrossSection", 4, [0], dbLevel)

\# PLASMA/Argon

iType = GuiDBM. SURFACEREACTION

iName = "PLASMA/Argon"

dbLevel = GuiDBM. MODEL

GuiDBM.AddItem (iType, iName, dbLevel)

GuiDBM.SetNotes (iType, iName, "", dbLevel)

GuiDBM.Set (iType, iName, "Type", "MULTI_STEP", dbLevel)

GuiDBM.Set (iType, iName, "Units", "SI", dbLevel)

GuiDBM.Set (iType, iName, "RateType", "GENERAL_RATE", dbLevel)

GuiDBM.AddReactionStep (iType, iName, "AR+->AR", dbLevel)

GuiDBM.SetListWithIndex(iType, iName, "ReactStep/Name", 1, " ", dbLevel)

GuiDBM.SetListWithIndex (iType, iName, "ReactStep/Notes", 1, " ", dbLevel)

GuiDBM.SetListWithIndex(iType, iName, "ReactStep/Forward_ConcentrationExponents", [1, 1], 1.000000, $\hookrightarrow$ dbLevel)

GuiDBM.SetListWithIndex(iType, iName, "ReactStep/Backward_ConcentrationExponents", [1, 1], 1.000000, $\hookrightarrow$ dbLevel)

GuiDBM.SetListWithIndex(iType, iName, "ReactStep/SurfaceCoverageModification", 1, 0, dbLevel)

GuiDBM.SetListWithIndex(iType, iName, "ReactStep/Type/Electro Chemical", 1, "No", dbLevel)

GuiDBM.SetListWithIndex(iType, iName, "ReactStep/Forward_Ap", 1, 1, dbLevel) 
GuiDBM.SetListWithIndex (iType, iName, "ReactStep/Forward_n", 1, 0, dbLevel)

GuiDBM.SetListWithIndex(iType, iName, "ReactStep/Forward_Ea/R", 1, 0, dbLevel)

GuiDBM.SetListWithIndex (iType, iName, "ReactStep/Forward_UserSub", 1, 0, dbLevel)

GuiDBM.SetListWithIndex(iType, iName, "ReactStep/Type/Sticking Coefficient", 1, "1", dbLevel)

GuiDBM.SetListWithIndex (iType, iName, "ReactStep/IonEnergyDependentRateFactor", 1, 0, dbLevel)

GuiDBM.SetListWithIndex (iType, iName, "ReactStep/IonEnergyDependentYieldFactor", 1, 0, dbLevel)

GuiDBM.SetListWithIndex(iType, iName, "ReactStep/IonAngleDependentYieldFactor", 1, 0, dbLevel)

GuiDBM.AddReactionStep (iType, iName, "AR*->AR", dbLevel)

GuiDBM.SetListWithIndex (iType, iName, "ReactStep/Name", 2, " , dbLevel)

GuiDBM.SetListWithIndex(iType, iName, "ReactStep/Notes", 2, " ", dbLevel)

GuiDBM.SetListWithIndex(iType, iName, "ReactStep/Forward_ConcentrationExponents", [2, 1], 1.000000, $\hookrightarrow \quad$ dbLevel)

GuiDBM.SetListWithIndex(iType, iName, "ReactStep/Backward_ConcentrationExponents", [2, 1], 1.000000, $\hookrightarrow$ dbLevel)

GuiDBM.SetListWithIndex(iType, iName, "ReactStep/SurfaceCoverageModification", 2, 0, dbLevel)

GuiDBM.SetListWithIndex (iType, iName, "ReactStep/Type/Electro Chemical", 2, "No", dbLevel)

GuiDBM.SetListWithIndex(iType, iName, "ReactStep/Forward_Ap", 2, 1, dbLevel)

GuiDBM.SetListWithIndex (iType, iName, "ReactStep/Forward_n", 2, 0, dbLevel)

GuiDBM.SetListWithIndex (iType, iName, "ReactStep/Forward_Ea/R", 2, 0, dbLevel)

GuiDBM.SetListWithIndex (iType, iName, "ReactStep/Forward_UserSub", 2, 0, dbLevel)

GuiDBM.SetListWithIndex (iType, iName, "ReactStep/Type/Sticking Coefficient", 2, "1", dbLevel)

GuiDBM.SetListWithIndex (iType, iName, "ReactStep/IonEnergyDependentRateFactor", 2, 0, dbLevel)

GuiDBM.SetListWithIndex (iType, iName, "ReactStep/IonEnergyDependentYieldFactor", 2, 0, dbLevel)

GuiDBM.SetListWithIndex(iType, iName, "ReactStep/IonAngleDependentYieldFactor", 2, 0, dbLevel)

GuiPT.Set("Application", "General")

GuiPT.Set ("Cav", 0)

GuiPT.Set ("Chem", 1)

GuiPT.Set ("Electr", 1)

GuiPT.Set ("Flow", 1)

GuiPT.Set ("VOF", 0)

GuiPT.Set ("Deform", 0)

GuiPT.Set ("Heat", 1)

GuiPT.Set ("Kinetic", 0)

GuiPT.Set ("MacP", 0)

GuiPT.Set ("Magnet", 0)

GuiPT.Set ("Plasma", 1)

GuiPT.Set ("Rad", 0)

GuiPT.Set ("Semi", 0)

GuiPT.Set ("Spray", 0)

GuiPT.Set ("Stress", 0)

GuiPT.Set ("Turb", 1)

GuiPT.Set ("Fluid2", 0)

GuiPT.Set ("Scalar", 0)

GuiMO.Set ("Shared/Title", "Symmetric PR -11/27/2017")

GuiMO.Set ("Shared/Polar", "Axisymmetric")

GuiMO.Set ("Shared/Transient_eval_method", "Transient")

GuiMO.Set ("Shared/FastTimestepping", 0)

GuiMO.Set("Shared/TimeStep_eval_method", "Standard")

GuiMO.Set ("Shared/NSteps", 8080)

GuiMO.Set ("Shared/DT", 7.382E-006)

GuiMO.Set ("Shared/TimeAccuracy", "Euler")

GuiMO.Set ("Shared/Gravity", 0)

GuiMO.Set ("Shared/Rotation", 0)

GuiMO.Set ("Shared/Chimera", 0)

GuimO.Set ("Flow/RefPressure", 100)

GuiMO.Set ("Flow/FanModel", 0)

GuiMO.Set ("Flow/MomentumResistanceModel", 0)

GuiMO.Set ("Flow/Swirl", 0)

GuiMO.Set("Electr/Options", "Electrostatics")

GuimO.Set ("Flow/Lorentz_Force", 0)

GuiMO.Set ("Flow/SlipWall", 0)

GuiMO.Set ("Flow/SlipInlet", 0)

GuiMO.Set ("Flow/FluidType", 0)

Guimo.Set ("Heat/Moving_Solid", 0)

Guimo.Set ("Heat/HeatExchModel", 0)

GuiMO.Set ("Turb/Model", "K Epsilon Family")

GuiMO.Set ("Turb/KEpsilonModel", "Standard")

GuiMO.Set ("Turb/WallFunction", "Standard Wall") 
GuiMO.Set("Turb/PrandtlNo", 0.9)

GuiMO.Set ("Turb/SchmidtNo", 0.9)

GuiMO.Set ("Turb/CompressibilityCorrection", 0)

GuiMO.Set("Chem/Chemistry_Model", "Gas Phase")

GuiMO.Set("Chem/SolverFor", "Species Mass Fractions")

GuiMO.Set ("Chem/GasPhaseReaction", 1)

GuiMO.Set ("Chem/ReactionName", "PLASMA/Argon")

GuiMO.Set ("Chem/DefaultSurfaceReaction", 0)

GuiMO.Set ("Chem/SolveCombustion", 0)

GuiMO.Set("Plasma/Model", "CCP")

GuiMO.Set("Plasma/IonMomentum", 0)

GuiMO.Set("Plasma/PositiveColumn", 0)

GuiMO.Set("Plasma/FixedCCPPower", "Off")

GuiMO.Set("Plasma/TimeStep_eval_method", "Time Steps per Cycle")

GuiMO.Set ("Plasma/TimeStepPerCycle", 20)

GuiMO.Set ("Plasma/CycleFreqSpecified", 0)

GuiMO.Set ("Plasma/MonteCarloTransport", 0)

GuiMO.Set("Plasma/EnableCCPAcceleration", 0)

GuiMO.Set ("Plasma/LossyDielectric", 0)

GuiMO.Set("Plasma/StrongBfield", 0)

GuiMO.Set ("Plasma/StochasticHeating", 0)

GuiMO.Set ("Plasma/LUT_Applied", 0)

GuiMO.Set("Plasma/EEDF_eval_method", "Maxwellian EEDF")

GuiMO.Set("Plasma/ElectronPressure", 1)

GuiMO.Set ("Plasma/TemperatureEquation", 1)

GuiMO.Set ("Plasma/JouleHeatingbyIconCurrent", 0)

GuiMO.Set ("Plasma/SurfaceHeatingbyIonImpact", 0)

GuiMO.Set("Adv/NeutralTimeStep", "Same as Shared Time Step")

GuimO.Set("Electr/Solver", "FVM Solver")

GuiMO.Set ("Electr/CircuitModel", 0)

GuiML.SetSpecies(["AR", "AR*", "AR+", "E"], 1)

GuiML.SetMixtures (["Argon", "ArgonIons"], 1)

objs=GuiVC. GetGeomObjByName ("Expansion")

GuiVC.Set(objs, "Shared/VC_Type", "Fluid")

GuiVC.Set(objs, "Shared/FluidUserInput", "User Input")

GuiVC.Set (objs, "Shared/MaterialName", "Air")

GuiVC.Set(objs, "Shared/Density_eval_method", "Ideal Gas Law")

GuiVC.Set(objs, "Shared/Viscosity_eval_method", "Mix Sutherland's Law")

GuiVC.Set(objs, "Heat/Cp_eval_method", "Constant")

GuiVC.Set(objs, "Heat/Cp", 520.43)

GuiVC.Set(objs, "Heat/k_eval_method", "Constant")

GuiVC.Set (objs, "Heat/k", 0.0263)

GuiVC.Set(objs, "Chem/Diffusivity_eval_method", "Schmidt Number")

GuiVC.Set (objs, "Chem/SchmidtNumber", 0.7)

GuiVC.Set(objs, "Chem/Mobility_eval_method", "From Diffusivity")

GuiVC.Set(objs, "Shared/EC_Property_eval_method", "Isotropic")

GuiVC.Set(objs, "Shared/ElectricalConductivity_eval_method", "Constant")

GuiVC.Set(objs, "Shared/ElectricalConductivity_Real", 1E-006)

GuiVC.Set(objs, "Shared/RelativePermittivity_eval_method", "Constant")

GuiVC.Set(objs, "Shared/RelativePermittivity", 1)

GuiVC.Set (objs, "Shared/RelativePermeability_eval_method", "Constant")

GuiVC.Set (objs, "Shared/RelativePermeability", 1)

GuiVC.Set(objs, "Electr/SpaceCharge_eval_method", "Density")

GuiVC.Set(objs, "Electr/SpaceCharge", 0)

GuiVC.Set(objs, "Plasma/CollisionFrequency_eval_method", "Constant")

GuiVC.Set(objs, "Plasma/CollisionFrequency", 20000000)

GuiVC.Set(objs, "Plasma/ElectronDiffusionCoefficient_eval_method", "Einstein's Equation")

GuiVC.Set(objs, "Chem/IonDiffusion_eval_method", "Same as for neutrals")

GuiVC.Set(objs, "Shared/FlowSource", 0)

GuiVC.Set (objs, "Shared/PorousMedia", 0)

GuiVC.Set (objs, "Shared/Momentum", 0)

GuiVC.Set (objs, "Shared/HeatSource", 0)

GuiVC.Set(objs, "Shared/Turbulence", 1)

GuiVC.Set(objs, "Shared/SpeciesSource", 0)

GuiVC.Set (objs, "Shared/PlasmaSource", 0)

objs=GuiVC. GetGeomObjByName ("Plenum")

GuiVC.Set(objs, "Shared/VC_Type", "Fluid") 
GuiVC.Set(objs, "Shared/FluidUserInput", "User Input")

GuiVC.Set(objs, "Shared/MaterialName", "Air")

GuiVC.Set(objs, "Shared/Density_eval_method", "Ideal Gas Law")

GuiVC.Set(objs, "Shared/Viscosity_eval_method", "Mix Sutherland's Law")

GuiVC.Set (objs, "Heat/Cp_eval_method", "Constant")

GuiVC.Set (objs, "Heat/Cp", 520.43)

GuiVC.Set(objs, "Heat/k_eval_method", "Constant")

GuiVC.Set (objs, "Heat/k", 0.0263)

GuiVC.Set(objs, "Chem/Diffusivity_eval_method", "Schmidt Number")

GuiVC.Set (objs, "Chem/SchmidtNumber", 0.7)

GuiVC.Set(objs, "Chem/Mobility_eval_method", "From Diffusivity")

GuiVC.Set (objs, "Shared/EC_Property_eval_method", "Isotropic")

GuiVC.Set (objs, "Shared/ElectricalConductivity_eval_method", "Constant")

GuiVC.Set(objs, "Shared/ElectricalConductivity_Real", 1E-006)

GuiVC.Set(objs, "Shared/RelativePermittivity_eval_method", "Constant")

GuiVC.Set(objs, "Shared/RelativePermittivity", 1)

GuiVC.Set(objs, "Shared/RelativePermeability_eval_method", "Constant")

GuiVC.Set (objs, "Shared/RelativePermeability", 1)

GuiVC.Set(objs, "Electr/SpaceCharge_eval_method", "Density")

GuiVC.Set (objs, "Electr/SpaceCharge", 0)

GuiVC.Set(objs, "Plasma/CollisionFrequency_eval_method", "Constant")

GuiVC.Set (objs, "Plasma/CollisionFrequency", 20000000)

GuiVC.Set(objs, "Plasma/ElectronDiffusionCoefficient_eval_method", "Einstein's Equation")

GuiVC.Set(objs, "Chem/IonDiffusion_eval_method", "Same as for neutrals")

GuiVC.Set (objs, "Shared/FlowSource", 0)

GuiVC.Set (objs, "Shared/PorousMedia", 0)

GuiVC.Set (objs, "Shared/Momentum", 0)

GuiVC.Set (objs, "Shared/HeatSource", 0)

GuiVC.Set(objs, "Shared/Turbulence", 1)

GuiVC.Set (objs, "Shared/SpeciesSource", 0)

GuiVC.Set(objs, "Shared/PlasmaSource", 0)

objs=GuiVC. GetGeomObjByName ("Tube")

GuiVC.Set (objs, "Shared/VC_Type", "Fluid")

GuiVC.Set (objs, "Shared/FluidUserInput", "User Input")

GuiVC.Set (objs, "Shared/MaterialName", "Air")

GuiVC.Set(objs, "Shared/Density_eval_method", "Ideal Gas Law")

GuiVC.Set(objs, "Shared/Viscosity_eval_method", "Mix Sutherland's Law")

GuiVC.Set (objs, "Heat/Cp_eval_method", "Constant")

GuiVC.Set (objs, "Heat/Cp", 520.43)

GuiVC.Set (objs, "Heat/k_eval_method", "Constant")

GuiVC.Set (objs, "Heat/k", 0.0263)

GuiVC.Set(objs, "Chem/Diffusivity_eval_method", "Schmidt Number")

GuiVC.Set (objs, "Chem/SchmidtNumber", 0.7)

GuiVC.Set(objs, "Chem/Mobility_eval_method", "From Diffusivity")

GuiVC.Set (objs, "Shared/EC_Property_eval_method", "Isotropic")

GuiVC.Set (objs, "Shared/ElectricalConductivity_eval_method", "Constant")

GuiVC.Set(objs, "Shared/ElectricalConductivity_Real", 1E-006)

GuiVC.Set(objs, "Shared/RelativePermittivity_eval_method", "Constant")

GuiVC.Set (objs, "Shared/RelativePermittivity", 1)

GuiVC.Set(objs, "Shared/RelativePermeability_eval_method", "Constant")

GuiVC.Set (objs, "Shared/RelativePermeability", 1)

GuiVC.Set (objs, "Electr/SpaceCharge_eval_method", "Density")

GuiVC.Set (objs, "Electr/SpaceCharge", 0)

GuiVC.Set(objs, "Plasma/CollisionFrequency_eval_method", "Constant")

GuiVC.Set (objs, "Plasma/CollisionFrequency", 20000000)

GuiVC.Set(objs, "Plasma/ElectronDiffusionCoefficient_eval_method", "Einstein's Equation")

GuiVC.Set(objs, "Chem/IonDiffusion_eval_method", "Same as for neutrals")

GuiVC.Set (objs, "Shared/FlowSource", 0)

GuiVC.Set (objs, "Shared/PorousMedia", 0)

GuiVC.Set (objs, "Shared/Momentum", 0)

GuiVC.Set (objs, "Shared/HeatSource", 0)

GuiVC.Set (objs, "Shared/Turbulence", 1)

GuiVC.Set(objs, "Shared/SpeciesSource", 0)

GuiVC.Set (objs, "Shared/PlasmaSource", 0)

objs=GuiVC. GetGeomObjByName ("Wall")

GuiVC.Set (objs, "Shared/VC_Type", "Solid")

GuiVC.Set (objs, "Shared/SolidFromDatabase", "ImportFromDatabase")

GuiVC.Set(objs, "Shared/MaterialName", "Dielectrics/Alumina") 
GuiVC.Set(objs, "Shared/Density_eval_method", "Constant")

GuiVC.Set(objs, "Shared/Density", 3950)

GuiVC.Set (objs, "Heat/Cp_eval_method", "Constant")

GuiVC.Set (objs, "Heat/Cp", 1000)

GuiVC.Set (objs, "Heat/k_eval_method", "Constant")

GuiVC.Set (objs, "Heat/k", 1)

GuiVC.Set(objs, "Shared/EC_Property_eval_method", "Isotropic")

GuiVC.Set(objs, "Shared/ElectricalConductivity_eval_method", "Dielectric")

GuiVC.Set(objs, "Shared/RelativePermittivity_eval_method", "Constant")

GuiVC.Set(objs, "Shared/RelativePermittivity", 9.5)

GuiVC.Set (objs, "Shared/RelativePermeability_eval_method", "Constant")

GuiVC.Set (objs, "Shared/RelativePermeability", 1)

GuiVC.Set (objs, "Electr/SpaceCharge_eval_method", "Total")

GuiVC.Set (objs, "Electr/SpaceCharge", 0)

GuiVC.Set(objs, "Shared/PorousMedia", 0)

GuiVC.Set (objs, "Shared/Momentum", 0)

GuiVC.Set (objs, "Shared/HeatSource", 0)

GuiVC.Set (objs, "Shared/PlasmaSource", 0)

objs=GuiBC. GetGeomObjByName ("Alumina")

GuiBC.Set ("Shared/BCSettingMode", "General")

GuiBC.Set (objs, "Shared/BC_Type", "Interface")

GuiBC.Set (objs, "Flow/U_eval_method", "Constant")

GuiBC.Set (objs, "Flow/U", 0)

GuiBC.Set (objs, "Flow/V_eval_method", "Constant")

GuiBC.Set (objs, "Flow/V", 0)

GuiBC.Set(objs, "Flow/Sub_Type", "No Slip")

GuiBC.Set (objs, "Heat/WallHeatSource", 0)

GuiBC.Set (objs, "Turb/RH_eval_method", "Constant")

GuiBC.Set (objs, "Turb/RH", 0)

GuiBC.Set (objs, "Chem/Sub_Type", "Surface Reaction")

GuiBC.Set (objs, "Chem/SurfReactName", "PLASMA/Argon")

GuiBC.Set(objs, "Plasma/ElectronT_eval_method", "Thermal Flux Balance")

GuiBC.Set (objs, "Plasma/ElectronND_eval_method", "Thermal Flux Balance")

GuiBC.Set (objs, "Plasma/SecEmisCoef_eval_method", "Constant")

GuiBC.Set(objs, "Plasma/Secondary_Emission_Coef", SEC)

GuiBC.Set(objs, "Electr/EP_eval_method", "Dielectric")

GuiBC.Set (objs, "Electr/Electroosmosis", 0)

objs=GuiBC. GetGeomObjByName ("Dielectric")

GuiBC.Set (objs, "Shared/BC_Type", "Wall")

GuiBC.Set (objs, "Heat/Sub_Type", "Adiabatic")

GuiBC.Set (objs, "Heat/SolidCellatWall", 0)

GuiBC.Set (objs, "Heat/WallHeatSource", 0)

GuiBC.Set(objs, "Electr/EP_eval_method", "Dielectric")

GuiBC.Set (objs, "Electr/Electroosmosis", 0)

objs=GuiBC.GetGeomObjByName ("FluidFluid")

GuiBC.Set (objs, "Shared/BC_Type", "Interface")

GuiBC.Set(objs, "Flow/Sub_Type", "Interface")

objs=GuiBC. GetGeomObjByName ("GND")

GuiBC.Set (objs, "Shared/BC_Type", "Wall")

GuiBC.Set (objs, "Flow/U_eval_method", "Constant")

GuiBC.Set (objs, "Flow/U", 0)

GuiBC.Set (objs, "Flow/V_eval_method", "Constant")

GuiBC.Set (objs, "Flow/V", 0)

GuiBC.Set(objs, "Flow/Sub_Type", "No Slip")

GuiBC.Set(objs, "Heat/Sub_Type", "Adiabatic")

GuiBC.Set(objs, "Heat/SolidCellatWall", 0)

GuiBC.Set (objs, "Heat/WallHeatSource", 0)

GuiBC.Set (objs, "Turb/RH_eval_method", "Constant")

GuiBC.Set (objs, "Turb/RH", 0)

GuiBC.Set (objs, "Chem/Sub_Type", "Surface Reaction")

GuiBC.Set (objs, "Chem/SurfReactName", "PLASMA/Argon")

GuiBC.Set (objs, "Plasma/ElectronT_eval_method", "Thermal Flux Balance")

GuiBC.Set (objs, "Plasma/ElectronND_eval_method", "Thermal Flux Balance")

GuiBC.Set (objs, "Plasma/SecEmisCoef_eval_method", "Constant")

GuiBC.Set (objs, "Plasma/Secondary_Emission_Coef", 0)

GuiBC.Set(objs, "Electr/EP_eval_method", "Fixed Potential")

GuiBC.Set (objs, "Electr/Voltage_eval_method", "Constant")

GuiBC.Set(objs, "Electr/Voltage_Re", 0) 
GuiBC.Set(objs, "Electr/Electroosmosis", 0)

objs=GuiBC. GetGeomObjByName ("GND_")

GuiBC.Set(objs, "Shared/BC_Type", "Wall")

GuiBC.Set(objs, "Heat/Sub_Type", "Adiabatic")

GuiBC.Set (objs, "Heat/SolidCellatWall", 0)

GuiBC.Set (objs, "Heat/WallHeatSource", 0)

GuiBC.Set(objs, "Electr/EP_eval_method", "Fixed Potential")

GuiBC.Set(objs, "Electr/Voltage_eval_method", "Constant")

GuiBC.Set (objs, "Electr/Voltage_Re", 0)

GuiBC.Set(objs, "Electr/Electroosmosis", 0)

objs=GuiBC. GetGeomObjByName ("Inlet")

GuiBC.Set (objs, "Shared/BC_Type", "Inlet")

GuiBC.Set (objs, "Flow/P_eval_method", "Constant")

GuiBC.Set (objs, "Flow/P", P_in)

GuiBC.Set(objs, "Flow/T_eval_method", "Constant")

GuiBC.Set(objs, "Flow/T", 300)

GuiBC.Set(objs, "Flow/Sub_Type", "Fixed Pressure")

GuiBC.Set(objs, "Turb/Sub_Type", "Fixed Value")

GuiBC.Set(objs, "Turb/K_eval_method", "Constant")

GuiBC.Set (objs, "Turb/K", 0)

GuiBC.Set(objs, "Turb/D_eval_method", "Constant")

GuiBC.Set (objs, "Turb/D", 0)

GuiBC.Set (objs, "Chem/SpeciesMassFraction_eval_method", "Constant")

GuiBC.Set(objs, "Chem/MixtureName", "Argon")

GuiBC.Set(objs, "Plasma/ElectronT_eval_method", "Fixed Gradient")

GuiBC.Set (objs, "Plasma/Gradient", 0)

GuiBC.Set(objs, "Plasma/ElectronND_eval_method", "Zero Gradient")

GuiBC.Set(objs, "Electr/EP_eval_method", "Surface Charge")

GuiBC.Set(objs, "Electr/SpecifyChargeDensity", 0)

GuiBC.Set(objs, "Electr/Surface_Charge_eval_method", "Constant")

GuiBC.Set (objs, "Electr/Charge", 0)

objs=GuiBC . GetGeomObjByName ("Outlet")

GuiBC.Set(objs, "Shared/BC_Type", "Outlet")

GuiBC.Set(objs, "Flow/P_eval_method", "Constant")

GuiBC.Set(objs, "Flow/P", P_exit)

GuiBC.Set(objs, "Flow/T_eval_method", "Constant")

GuiBC.Set (objs, "Flow/T", 300)

GuiBC.Set(objs, "Flow/Sub_Type", "Fixed Pressure")

GuiBC.Set(objs, "Turb/K_eval_method", "Constant")

GuiBC.Set (objs, "Turb/K", 0)

GuiBC.Set(objs, "Turb/D_eval_method", "Constant")

GuiBC.Set (objs, "Turb/D", 0)

GuiBC.Set(objs, "Chem/SpeciesMassFraction_eval_method", "Constant")

GuiBC.Set (objs, "Chem/MixtureName", "Argon")

GuiBC.Set(objs, "Plasma/ElectronT_eval_method", "Fixed Gradient")

GuiBC.Set (objs, "Plasma/Gradient", 0)

GuiBC.Set(objs, "Plasma/ElectronND_eval_method", "Zero Gradient")

GuiBC.Set(objs, "Electr/EP_eval_method", "Surface Charge")

GuiBC.Set (objs, "Electr/SpecifyChargeDensity", 0)

GuiBC.Set (objs, "Electr/Surface_Charge_eval_method", "Constant")

GuiBC.Set (objs, "Electr/Charge", 0)

objs=GuiBC . GetGeomObjByName ("RF")

GuiBC.Set (objs, "Shared/BC_Type", "Wall")

GuiBC.Set(objs, "Heat/Sub_Type", "Adiabatic")

GuiBC.Set (objs, "Heat/SolidCellatWall", 0)

GuiBC.Set (objs, "Heat/WallHeatSource", 0)

GuiBC.Set(objs, "Electr/EP_eval_method", "Fixed Potential")

GuiBC.Set(objs, "Electr/Voltage_eval_method", "V = V1 + V0*Sin(360*f*t + phi)")

GuiBC.Set (objs, "Electr/VO", V_RF)

GuiBC.Set (objs, "Electr/V1", 0)

GuiBC.Set(objs, "Electr/Frequency", 13560000)

GuiBC.Set (objs, "Electr/Phi", 0)

GuiBC.Set(objs, "Electr/Electroosmosis", 0)

objs=GuiBC. GetGeomObjByName ("Symmetry")

GuiBC.Set (objs, "Shared/BC_Type", "Symmetry")

objs=GuiIC . GetGeomObjByName ("Expansion")

GuiIC.Set("Shared/IC_Source", "Constant")

GuiIC.Set("Shared/IC_Setting", "Volume by Volume") 
GuiIC.Set (objs, "Shared/T_eval_method", "Constant")

GuiIC.Set (objs, "Shared/T", 300)

GuiIC.Set(objs, "Flow/U_eval_method", "Constant")

GuiIC.Set (objs, "Flow/U", 0)

GuiIC.Set (objs, "Flow/V_eval_method", "Constant")

GuiIC.Set (objs, "Flow/V", 0)

GuiIC.Set (objs, "Flow/P_eval_method", "Constant")

GuilC.Set(objs, "Flow/P", P_exit)

GuiIC.Set (objs, "Turb/K_eval_method", "Constant")

GuiIC.Set (objs, "Turb/K", 0)

GuiIC.Set (objs, "Turb/D_eval_method", "Constant")

GuiIC.Set (objs, "Turb/D", 0)

GuiIC.Set (objs, "Chem/MixtureName", "Argon")

GuiIC.Set (objs, "Plasma/Te_eval_method", "Constant")

GuiIC.Set (objs, "Plasma/Te", 1)

GuiIC.Set (objs, "Plasma/Ne_eval_method", "Constant")

GuiIC.Set (objs, "Plasma/Ne", 1000)

GuiIC.Set (objs, "Electr/Voltage", 0)

objs=GuiIC. GetGeomObjByName ("Plenum")

GuiIC.Set (objs, "Shared/T_eval_method", "Constant")

GuiIC.Set (objs, "Shared/T", 300)

GuiIC.Set (objs, "Flow/U_eval_method", "Constant")

GuiIC.Set (objs, "Flow/U", 0)

GuiIC.Set (objs, "Flow/V_eval_method", "Constant")

GuiIC.Set (objs, "Flow/V", 0)

GuiIC.Set (objs, "Flow/P_eval_method", "Constant")

GuiIC.Set(objs, "Flow/P", P_in)

GuiIC.Set (objs, "Turb/K_eval_method", "Constant")

GuiIC.Set (objs, "Turb/K", 0)

GuiIC.Set (objs, "Turb/D_eval_method", "Constant")

GuiIC.Set (objs, "Turb/D", 0)

GuiIC.Set (objs, "Chem/MixtureName", "Argon")

GuiIC.Set (objs, "Plasma/Te_eval_method", "Constant")

GuiIC.Set (objs, "Plasma/Te", 1)

GuiIC.Set(objs, "Plasma/Ne_eval_method", "Constant")

GuiIC.Set (objs, "Plasma/Ne", 100)

GuiIC.Set (objs, "Electr/Voltage", 0)

objs=GuiIC. GetGeomObjByName ("Tube")

GuiIC.Set (objs, "Shared/T_eval_method", "Constant")

GuiIC.Set (objs, "Shared/T", 300)

GuiIC.Set (objs, "Flow/U_eval_method", "Constant")

GuiIC.Set (objs, "Flow/U", 0)

GuiIC.Set (objs, "Flow/V_eval_method", "Constant")

GuiIC.Set (objs, "Flow/V", 0)

GuiIC.Set (objs, "Flow/P_eval_method", "Constant")

GuiIC.Set (objs, "Flow/P", 100)

GuiIC.Set (objs, "Turb/K_eval_method", "Constant")

GuiIC.Set (objs, "Turb/K", 0)

GuiIC.Set (objs, "Turb/D_eval_method", "Constant")

GuiIC.Set (objs, "Turb/D", 0)

GuiIC.Set (objs, "Chem/MixtureName", "ArgonIons")

GuiIC.Set (objs, "Plasma/Te_eval_method", "Constant")

GuilC.Set (objs, "Plasma/Te", 2.5)

GuiIC.Set (objs, "Plasma/Ne_eval_method", "Constant")

GuiIC.Set (objs, "Plasma/Ne", 1E+016)

GuiIC.Set(objs, "Electr/Voltage", 0)

objs=GuiIC. GetGeomObjByName ("Wall")

GuiIC.Set(objs, "Shared/T_eval_method", "Constant")

GuiIC.Set (objs, "Shared/T", 300)

GuiIC.Set (objs, "Electr/Voltage", 0)

GuiSC.Set ("Adv/FastCCPSolver", 0)

GuiSC.Set ("Iter/Max_Iteration", 12)

GuiSC.Set ("Iter/Convergence_Crit", 1E-005)

GuiSC.Set ("Iter/Min_Residual", 1E-018)

GuiSC.Set ("Iter/MassBalanceCriteria", 0)

GuiSC.Set ("Iter/EnergyBalanceCriteria", 0)

GuiSC.Set ("Solvers/Advanced", 1)

GuiSC.Set ("Adv/BufferedOutput", 0) 
GuiSC.Set("Adv/HigherAccuracy", 0)

GuiSC.Set ("Adv/MinimumFaceAngleSkew", 0)

GuiSC.Set("Adv/GradientCalcMethod", "Green-Gauss Methodology")

GuiSC.Set("Adv/GreenGaussMethod", "Cell-To-Face")

GuiSC.Set ("Adv/OverrideSolvers", 0)

GuiSC.Set("Spatial/Velocity_diff_method", "Upwind")

GuiSC.Set("Solvers/Velocity_Scheme", "CGS")

GuiSC.Set("PreConditioner/Velocity_PreConditioner_Scheme", "ILU(k)")

GuiSC.Set("Solvers/Velocity_Sweeps", 50)

GuiSC.Set("Solvers/Velocity_Criterion", 0.0001)

GuiSC.Set("PreConditioner/Velocity_Fill_Levels", 0)

GuiSC.Set ("PreConditioner/Velocity_Expected_Fill", 1)

GuiSC.Set("PreConditioner/Velocity_Zeros_on_Diagonal", "No")

GuiSC.Set("Solvers/PCorrection_Scheme", "AMG")

GuiSC.Set("PreConditioner/PCorrection_PreConditioner_Scheme", "None")

GuiSC.Set("Solvers/PCorrection_Sweeps", 50)

GuiSC.Set ("Solvers/PCorrection_Criterion", 0.1)

GuiSC.Set("Relax/Velocity", 0.2)

GuiSC.Set("Relax/PCorrection", 0.2)

GuiSC.Set("Relax/Pressure", 1)

GuiSC.Set("Relax/Density", 1)

GuiSC.Set("Relax/Viscosity", 1)

GuiSC.Set ("Limits/U_Min", -1E+020)

GuiSC.Set ("Limits/U_Max", 1E+020)

GuiSC.Set("Limits/V_Min", -1E+020)

GuiSC.Set ("Limits/V_Max", 1E+020)

GuiSC.Set("Limits/P_Min", -1E+020)

GuiSC.Set ("Limits/P_Max", 1E+020)

GuiSC.Set("Limits/Density_Min", 1E-006)

GuiSC.Set ("Limits/Density_Max", 1E+020)

GuiSC.Set("Limits/Viscosity_Min", 1E-010)

GuiSC.Set("Limits/Viscosity_Max", 100)

GuiSC.Set ("Adv/CutDiffusionAtInlets", 0)

GuiSC.Set ("Adv/Flow_CFL_Relaxation", 0)

GuiSC.Set("Spatial/Enthalpy_diff_method", "Upwind")

GuiSC.Set("Solvers/Enthalpy_Scheme", "CGS")

GuiSC.Set("PreConditioner/Enthalpy_PreConditioner_Scheme", "ILU(k)")

GuiSC.Set("Solvers/Enthalpy_Sweeps", 50)

GuiSC.Set("Solvers/Enthalpy_Criterion", 0.0001)

GuiSC.Set("PreConditioner/Enthalpy_Fill_Levels", 0)

GuiSC.Set ("PreConditioner/Enthalpy_Expected_Fill", 1)

GuiSC.Set("PreConditioner/Enthalpy_Zeros_on_Diagonal", "No")

GuiSC.Set("Relax/Enthalpy", 0.05)

GuiSC.Set ("Relax/Temperature", 1)

GuiSC.Set("Limits/Enthalpy_Min", -1E+020)

GuiSC.Set ("Limits/Enthalpy_Max", 1E+020)

GuiSC.Set ("Limits/Temperature_Min", 1E-010)

GuiSC.Set ("Limits/Temperature_Max", 5000)

GuiSC.Set ("Adv/CutDiffusionHeat", 0)

GuiSC.Set ("Adv/Heat_CFL_Relaxation", 0)

GuiSC.Set ("Adv/Heat_VISCOUS_DISSIPATION", 1)

GuiSC.Set ("Adv/HeatTempInterpolation", "Apply All BCs")

GuiSC.Set("Spatial/Turbulence_diff_method", "Upwind")

GuiSC.Set ("Solvers/Turbulence_Scheme", "CGS")

GuiSC.Set ("PreConditioner/Turbulence_PreConditioner_Scheme", "ILU(k)")

GuiSC.Set ("Solvers/Turbulence_Sweeps", 50)

GuiSC.Set("Solvers/Turbulence_Criterion", 0.0001)

GuiSC.Set ("PreConditioner/Turbulence_Fill_Levels", 0)

GuiSC.Set ("PreConditioner/Turbulence_Expected_Fill", 1)

GuiSC.Set ("PreConditioner/Turbulence_Zeros_on_Diagonal", "No")

GuiSC.Set ("Relax/Turbulence", 0.2)

GuiSC.Set("Limits/KineticEnergy_Min", 0)

GuiSC.Set ("Limits/KineticEnergy_Max", 1E+020)

GuiSC.Set ("Limits/DissipationRate_Min", 0)

GuiSC.Set("Limits/DissipationRate_Max", 1E+020)

GuiSC.Set ("Adv/CutDiffusionTurb", 0)

GuiSC.Set ("Adv/Turb_CFL_Relaxation", 0)

GuiSC.Set("Spatial/Species_diff_method", "Upwind") 
GuiSC.Set ("Solvers/Species_Scheme", "CGS")

GuiSC.Set("PreConditioner/Species_PreConditioner_Scheme", "ILU(k)")

GuiSC.Set ("Solvers/Species_Sweeps", 50)

GuiSC.Set("Solvers/Species_Criterion", 0.0001)

GuiSC.Set ("PreConditioner/Species_Fill_Levels", 0)

GuiSC.Set ("PreConditioner/Species_Expected_Fill", 1)

GuiSC.Set ("PreConditioner/Species_Zeros_on_Diagonal", "No")

GuiSC.Set ("Relax/SpeciesLinearRelax", 1)

GuiSC.Set ("Limits/Species_Min", 0)

GuiSC.Set ("Limits/Species_Max", 1)

GuiSC.Set ("Adv/CutDiffusionChem", 1)

GuiSC.Set ("Adv/CutDiffusionChemAcceleration", 0)

GuiSC.Set ("Adv/Chem_CFL_Relaxation", 0)

GuiSC.Set ("Adv/SpeciesConservationEnforced", 0)

GuiSC.Set("Spatial/Te_diff_method", "Exponential")

GuiSC.Set("Spatial/Ne_diff_method", "Exponential")

GuiSC.Set ("Solvers/Te_Scheme", "CGS")

GuiSC.Set ("PreConditioner/Te_PreConditioner_Scheme", "ILU(k)")

GuiSC.Set ("Solvers/Te_Sweeps", 50)

GuiSC.Set ("Solvers/Te_Criterion", 0.0001)

GuiSC.Set ("PreConditioner/Te_Fill_Levels", 0)

GuiSC.Set ("PreConditioner/Te_Expected_Fill", 1)

GuiSC.Set ("PreConditioner/Te_Zeros_on_Diagonal", "No")

GuiSC.Set ("Solvers/Ne_Scheme", "CGS")

GuiSC.Set ("PreConditioner/Ne_PreConditioner_Scheme", "ILU(k)")

GuiSC.Set ("Solvers/Ne_Sweeps", 50)

GuiSC.Set ("Solvers/Ne_Criterion", 0.0001)

GuiSC.Set ("PreConditioner/Ne_Fill_Levels", 0)

GuiSC.Set ("PreConditioner/Ne_Expected_Fill", 1)

GuiSC.Set ("PreConditioner/Ne_Zeros_on_Diagonal", "No")

GuiSC.Set ("Relax/TeNe", 3E-005)

GuiSC.Set ("Relax/TeNe_Linear", 0.2)

GuiSC.Set ("Relax/Ne", 3E-005)

GuiSC.Set ("Relax/Ne_Linear", 0.8)

GuiSC.Set("Relax/PositiveIons", 2E-005)

GuiSC.Set ("Relax/NegativeIons", 2E-005)

GuiSC.Set ("Relax/Neutralspecies", 2E-005)

GuiSC.Set ("Relax/PositiveIonsLinear", 0.9)

GuiSC.Set ("Relax/NegativeIonsLinear", 0.9)

GuiSC.Set ("Relax/NeutralspeciesLinear", 0.9)

GuiSC.Set ("Limits/Te_Min", 0.1)

GuiSC.Set ("Limits/Te_Max", 60)

GuiSC.Set ("Limits/Ne_Min", 1)

GuiSC.Set ("Limits/Ne_Max", 1E+020)

GuiSC.Set ("Adv/RampingElectronHeating", 1)

GuiSC.Set ("Adv/CCP_Iterations", 5)

GuiSC.Set ("Solvers/ElectricPotential_Scheme", "CGS")

GuiSC.Set ("PreConditioner/ElectricPotential_PreConditioner_Scheme", "ILU(k)")

GuiSC.Set ("Solvers/ElectricPotential_Sweeps", 500)

GuiSC.Set ("Solvers/ElectricPotential_Criterion", 0.0001)

GuiSC.Set ("PreConditioner/ElectricPotential_Fill_Levels", 0)

GuiSC.Set ("PreConditioner/ElectricPotential_Expected_Fill", 1)

GuiSC.Set ("PreConditioner/ElectricPotential_Zeros_on_Diagonal", "No")

GuiSC.Set ("Relax/ElectricPotential", 0.0002)

GuiSC.Set ("Limits/ElectricPotential_Min", -1E+020)

GuiSC.Set ("Limits/ElectricPotential_Max", 1E+020)

GuiOut.Set("Output/Output_Mode", "Timestep")

GuiOut.Set("Output/Output_Begin_TStep", 0)

GuiOut.Set ("Output/Output_End_TStep", 10000000)

GuiOut.Set("Output/Output_Interval", 101)

GuiOut.Set("Output/Output_Location", "Unique Filename")

GuiOut.Set ("Print/SetResidualFrequency", 0)

GuiOut.Set ("Output/CGNSOutput", 0)

GuiOut.Set ("Output/RADTHERMOutput", 0)

GuiOut.Set ("Print/Diagnostics", 0)

GuiOut.Set ("Output/RestartOverrideGeneral", 0)

GuiOut.Set ("Output/RestartSolution", 1)

GuiOut.Set ("Output/GraphicsOutputFile", "DTF") 
GuiOut.Set ("Output/GraphicOverrideGeneral", 0)

GuiOut.Set("Graphic/Density", 1)

GuiOut.Set ("Graphic/T", 1)

GuiOut.Set ("Graphic/T0", 1)

GuiOut.Set ("Graphic/ElectricConductivity", 0)

GuiOut.Set ("Graphic/RelativePermeability", 0)

GuiOut.Set ("Graphic/RelativePermittivity", 0)

GuiOut.Set ("Output/SummaryOverrideGeneral", 0)

GuiOut.Set ("Output/WriteGIDKeyMaps", 0)

GuiOut.Set ("Output/MonitorPointOverrideGeneral", 0)

GuiOut.Set ("Monitor/Monitor_Points", 1)

GuiOut.Set ("Monitor/Monitor_Def_Method", "User Input")

GuiOut.SetArraySize("Monitor/TotalMonitorPoints", 5)

GuiOut.SetArray ("Monitor/MP_X", 1, 0.011)

GuiOut.SetArray ("Monitor/MP_Y", 1, 0)

GuiOut.SetArraySize("Monitor/TotalMonitorPoints", 5)

GuiOut.SetArray ("Monitor/MP_X", 2, 0.02)

GuiOut.SetArray ("Monitor/MP_Y", 2, 0)

GuiOut.SetArraySize ("Monitor/TotalMonitorPoints", 5)

GuiOut.SetArray ("Monitor/MP_X", 3, 0.011)

GuiOut.SetArray ("Monitor/MP_Y", 3, 0.002)

GuiOut.SetArraySize("Monitor/TotalMonitorPoints", 5)

GuiOut.SetArray ("Monitor/MP_X", 4, -0.005)

GuiOut.SetArray ("Monitor/MP_Y", 4, 0.01)

GuiOut.SetArraySize("Monitor/TotalMonitorPoints", 5)

GuiOut.SetArray ("Monitor/MP_X", 5, 0.05)

GuiOut.SetArray ("Monitor/MP_Y", 5, 0.01)

GuiOut.Set ("Monitor/RHO", 1)

GuiOut.Set ("Monitor/Location", 0)

GuiOut.Set ("Output/MonitorPlaneOverrideGeneral", 0)

GuiOut.Set("Print/BC_Integral_Output", 0)

GuiOut.Set ("Output/UserSub_UOUT", 0)

GuiOut.Set ("Output/UserSub_UREAD_DTF", 0)

GuiOut.Set ("Output/UserSub_UWRITE_DTF", 0)

GuiOut.Set ("Print/Force_Summary", 0)

GuiOut.Set ("Print/MassFlow_Sum", 1)

GuiOut.Set ("Flow/Monitor_IQ_Mass_Imbalance", 0)

GuiOut.Set ("Flow/MFSOutput", "Individual")

GuiOut.Set ("Print/Flow_Interface_Summary", 1)

GuiOut.Set ("Graphic/Velocity", 1)

GuiOut.Set ("Graphic/VelocityMagnitude", 1)

GuiOut.Set ("Graphic/P", 1)

GuiOut.Set ("Graphic/PO", 1)

GuiOut.Set ("Graphic/LaminarViscosity", 0)

GuiOut.Set ("Graphic/MachNumber", 1)

GuiOut.Set ("Graphic/Vorticity", 0)

GuiOut.Set ("Graphic/StreamFunction", 0)

GuiOut.Set ("Graphic/StrainRate", 0)

GuiOut.Set ("Graphic/CFLNumber", 0)

GuiOut.Set ("Graphic/Porosity", 0)

GuiOut.Set ("Graphic/WallViscousStress", 0)

GuiOut.Set ("Graphic/WallViscousStressMagnitude", 0)

GuiOut.Set ("Graphic/WallShearStress", 0)

GuiOut.Set ("Graphic/WallShearStressMagnitude", 0)

GuiOut.Set ("Graphic/SkinFrictionCoeff", 0)

GuiOut.Set ("Graphic/PressureCoefficient", 0)

GuiOut.Set ("Graphic/X-DirectionVelocityResidual", 0)

GuiOut.Set ("Graphic/Y-DirectionVelocityResidual", 0)

GuiOut.Set ("Graphic/PressureResidual", 0)

GuiOut.Set ("Monitor/U", 1)

GuiOut.Set ("Monitor/V", 1)

GuiOut.Set ("Monitor/W", 0)

GuiOut.Set ("Monitor/P", 1)

GuiOut.Set("Monitor/PTotal", 0)

GuiOut.Set ("Monitor/MU", 0)

GuiOut.Set ("Monitor/Mach", 0)

GuiOut.Set ("Monitor/Vorticity", 0)

GuiOut.Set("Monitor/Stream_Function", 0) 
GuiOut.Set("Monitor/Strain_Rate", 0)

GuiOut.Set("Monitor_Plane/Mass_Flux", 0)

GuiOut.Set("Monitor_Plane/Volumetric_Flux", 0)

GuiOut.Set("Monitor_Plane/AvgPress", 0)

GuiOut.Set ("Monitor_Plane/VelMag", 0)

GuiOut.Set ("Monitor_Plane/VelComp", 0)

GuiOut.Set("Monitor_Plane/Density", 0)

GuiOut.Set ("Print/HeatTransSum", 0)

GuiOut.Set("Graphic/StaticEnthalpy", 1)

GuiOut.Set ("Graphic/SpecificHeat", 0)

GuiOut.Set("Graphic/ThermalConductivity", 0)

GuiOut.Set ("Graphic/ThermalConductivityPorous", 0)

GuiOut.Set ("Graphic/WallHeatFlux", 0)

GuiOut.Set ("Graphic/HeatResidual", 0)

GuiOut.Set ("Graphic/PrandtINumber", 0)

GuiOut.Set ("Graphic/NusseltNumber", 0)

GuiOut.Set ("Graphic/HeatTransferCoeff", 0)

GuiOut.Set ("Monitor/T", 1)

GuiOut.Set("Monitor/H", 0)

GuiOut.Set("Monitor/COND", 0)

GuiOut.Set ("Monitor/Cp", 0)

GuiOut.Set ("Monitor_Plane/Avg_Temp", 0)

GuiOut.Set ("Graphic/TurbKineticEnergy", 1)

GuiOut.Set ("Graphic/TurbDissipationRate", 0)

GuiOut.Set ("Graphic/TurbViscosity", 0)

GuiOut.Set ("Graphic/EffectiveVisc", 0)

GuiOut.Set ("Graphic/Y+", 0)

GuiOut.Set ("Graphic/TurbKineticEnergyResidual", 0)

GuiOut.Set ("Graphic/TurbDissipationRateResidual", 0)

GuiOut.Set ("Monitor/TKE", 0)

GuiOut.Set ("Monitor/TD", 0)

GuiOut.Set ("Monitor/TurbMu", 0)

GuiOut.Set ("Monitor/Y+", 0)

GuiOut.Set("Print/SpeciesSummary", 0)

GuiOut.Set ("Print/SurfReactSum", 0)

GuiOut.Set ("Monitor/MassFractions", 1)

GuiOut.Set ("Graphic/MassFractions", 1)

GuiOut.Set ("Graphic/MoleFractions", 0)

GuiOut.Set ("Graphic/ReactionRate", 0)

GuiOut.Set("Graphic/SpeciesFlux", 0)

GuiOut.Set("Graphic/SpeciesDiffusivity", 1)

GuiOut.Set ("Graphic/SpeciesThermDiff", 0)

GuiOut.Set ("Graphic/DepositionRate", 0)

GuiOut.Set ("Graphic/NumDensity", 1)

GuiOut.Set ("Graphic/IonMobility", 1)

GuiOut.Set ("Graphic/SpeciesResidual", 0)

GuiOut.Set("Print/FeatureScaleCoupling", 0)

GuiOut.Set ("Output/CCP_Cycle_Frequency", 500)

GuiOut.Set ("Print/PlasmaHeatingBalance", 0)

GuiOut.Set ("Print/CCPCycleAveragedSummary", 1)

GuiOut.Set ("Print/PlasmaCPPPrintFrequency", 1)

GuiOut.Set ("Print/MonitorVJatBoundaryPoints", 0)

GuiOut.Set ("Monitor/Te", 1)

GuiOut.Set ("Monitor/Ne", 1)

GuiOut.Set ("Graphic/ElectronDensity", 1)

GuiOut.Set ("Graphic/ElectronTemperature", 1)

GuiOut.Set("Graphic/ElectronMobility", 1)

GuiOut.Set("Graphic/ElectronFlux", 0)

GuiOut.Set ("Graphic/Power", 0)

GuiOut.Set ("Graphic/AvgElectDensity", 1)

GuiOut.Set ("Graphic/AvgElectTemp", 1)

GuiOut.Set ("Graphic/AvgPower", 1)

GuiOut.Set("Graphic/AvgPotential", 1)

GuiOut.Set ("Graphic/AvgReactionRate", 0)

GuiOut.Set ("Graphic/SpeciesNumDensity", 1)

GuiOut.Set ("Graphic/AvgElectField", 1)

GuiOut.Set ("Graphic/AvgSpeciesFluxes", 0)

GuiOut.Set ("Adv/CCPAnalysisInColumns", 0) 
GuiOut.Set("Print/Charge_Summary", 0)

GuiOut.Set ("Graphic/ElectricPotential", 1)

GuiOut.Set ("Graphic/ElectrostaticPressure", 0)

GuiOut.Set("Graphic/ElectricFieldVector", 1)

GuiOut.Set ("Graphic/SurfaceCharge", 0)

GuiOut.Set ("Graphic/SpaceCharge", 1)

GuiOut.Set("Graphic/SolidElectricConductivity", 0)

GuiOut.Set ("Graphic/E-Flux", 0)

GuiOut.Set("Monitor/Electric_Potential", 1)

GuiOut.Set("Monitor/Electric_Field", 0)

GuiOut.Set ("Monitor/Current", 0)

GuiRun.Set ("Shared/ParallelRun", 0)

GuiRun.Set("Shared/Precision", "Double Precision")

GuiRun.Set ("Shared/UserSharedLibrary", "libUserAce.DLL") 